\title{
Déformations tardi-grenvilliennes dans la Ceinture parautochtone (Province de Grenville centrale) : contraintes géochronologiques par couplage de méthodes $\mathrm{U} / \mathrm{Pb}$ de haute résolution spatiale et de haute précision
}

\begin{tabular}{|r|l|}
\hline Journal: & Canadian Journal of Earth Sciences \\
\hline Manuscript ID & cjes-2017-0129.R2 \\
\hline Manuscript Type: & Article \\
\hline Date Submitted by the Author: & 26 -Jan-2018 \\
\hline Complete List of Authors: & $\begin{array}{l}\text { Jannin, Sophie; Polytechnique Montréal, Département des génies civil, } \\
\text { géologique et des mines } \\
\text { Gervais, Felix; Polytechnique Montréal, Département des génies civil, } \\
\text { géologique et des mines } \\
\text { Moukhsil, Abdelali; Ministère I'Energie et des Resources naturelles, } \\
\text { Direction du Bureau de I'exploration Géologique du Québec } \\
\text { Augland, Lars Eivind; Universitetet i Oslo Det Matematisk- } \\
\text { naturvitenskapelige Fakultet, Centre for Earth Evolution and Dynamics } \\
\text { Crowley, James; Department of Geosciences }\end{array}$ \\
\hline $\begin{array}{r}\text { Is the invited manuscript for } \\
\text { consideration in a Special } \\
\text { Issue? : }\end{array}$ & $\begin{array}{l}\text { N/A } \\
\text { Keyword: }\end{array}$ \\
\hline Géochronologie U/Pb, Métamorphisme \\
\hline
\end{tabular}




\section{Déformations tardi-grenvilliennes dans la Ceinture parautochtone (Province}

\section{de Grenville centrale) : contraintes géochronologiques par couplage de méthodes $\mathrm{U} / \mathrm{Pb}$ de haute résolution spatiale et de haute précision}

Auteurs : Sophie Jannin ${ }^{1}$, Félix Gervais ${ }^{2}$, Abdelali Moukhsil ${ }^{3}$, Lars Eiving Augland ${ }^{4 a}$, James L. Crowley $^{5}$

Auteur-correspondant :

${ }^{1}$ Département des génies civil, géologique et des mines, Polytechnique Montréal, C.P. 6079, succ. Centre-Ville - Montréal (QC), H3C 3A7, Canada. sophie.jannin@gmail.com, +1 514-2311274.

2 Département des génies civil, géologique et des mines, Polytechnique Montréal, C.P. 6079, succ. Centre-Ville - Montréal (QC), H3C 3A7, Canada. felix.gervais@polymtl.ca

${ }^{3}$ Direction du Bureau de la connaissance géoscientifique du Québec, Ministère de l'Énergie et des Ressources naturelles, 5700, $4^{\text {ème }}$ Avenue Ouest, bureau D-307, Québec (QC), G1H 6R1, Canada. Abdelali.Moukhsil@mern.gouv.qc.ca

${ }^{4}$ GEOTOP, Université du Québec à Montréal, CP 8888, Succ. Centre-Ville, Montréal (QC), H3C 3P8, Canada.augland.lars_eivind@courrier.uqam.ca*

${ }^{5}$ Departement of Geosciences, 1910 University Dr., Boise State University, Boise (ID), 837251535, USA.jimcrowley@boisestate.edu

${ }^{a}$ affiliation actuelle : Center for Earth Evolution and Dynamics (CEED), University of Oslo, P.O. Box 1028 Blindern, 0315 Oslo, Norway. 1.e.augland@geo.uio.no 


\section{Résumé}

Dans la Ceinture parautochtone de la Province de Grenville centrale (réservoir Manicouagan), la datation des structures révèle que l'orogène grenvillien a oblitéré les fabriques antérieures.

Ainsi, les structures D1 et D2 indiquent un cisaillement inverse à vergence nord, avec respectivement des linéations SSE et SSO. Les structures D3 indiquent un cisaillement obliquenormal à vergence OSO.

Une stratégie innovatrice combinant la géochronologie $\mathrm{U} / \mathrm{Pb}$ sur zircon par ablation laser (LAICP-MS) et par abrasion chimique par dilution isotopique (CA-ID-TIMS) contraint l'âge de ces déformations. Les surcroissances internes et externes des grains de zircon d'un leucosome synD2, indiquant deux périodes de croissance, sont interprétées respectivement comme les âges de $\mathrm{D} 1$ et $\mathrm{D} 2$. Le groupe 1 donne un âge ${ }^{206} \mathrm{~Pb} /{ }^{207} \mathrm{~Pb}$ de $1008 \pm 18$ Ma (LA-ICP-MS) et un âge concordia de $1002 \pm 2 \mathrm{Ma}$ (CA-ID-TIMS). Le groupe 2 donne un âge ${ }^{206} \mathrm{~Pb} /{ }^{207} \mathrm{~Pb}$ de $995 \pm 21 \mathrm{Ma}$ (LA-ICP-MS) et un âge concordia de $986 \pm 4$ Ma (CA-ID-TIMS). Une pegmatite tardi-D2 donne un âge ${ }^{206} \mathrm{~Pb} /{ }^{207} \mathrm{~Pb}$ de $961 \pm 22 \mathrm{Ma}$ (LA-ICP-MS). L'âge de D1 coïncide avec celui du groupe $1 \mathrm{du}$ leucosome, soit 1002 Ma. En effet, le zircon ne peut croître avant l'anatexie, or ce groupe possède les caractéristiques d'une croissance en présence de fusion partielle et un rubanement migmatitique définit la S1. L'âge de D2 est contraint à 986-961 Ma par la cristallisation du leucosome syn-D2 coïncidant au groupe 2, et celle de la pegmatite tardi-D2.

Ainsi, dans la Ceinture parautochtone centrale près de l'ABT, la première déformation étant grenvillienne, aucune évidence d'évènement tectono-métamorphique pré-grenvillien n'est préservée. 
Mots clés : Province de Grenville, Ceinture parautochtone, Phases de déformation, Géochronologie U/Pb, Métamorphisme

\section{Introduction}

Lors d'une orogénèse impliquant un continent, le socle se fait éventuellement chevaucher et est incorporé dans le cœur d'un orogène. Distinguer les structures et textures métamorphiques formées lors de cette orogenèse de celles formées lors d'évènements tectoniques antérieurs est un grand défi qui requiert une approche multidisciplinaire. Plusieurs études ont lié des réactions métamorphiques à des zones de croissance spécifiques de zircon ou de monazite par leur texture (p. ex. Vavra et al., 1990; Gervais et Hynes, 2013) ou leur composition chimique (p. ex. Rubatto et al., 2001, 2006; Foster et al., 2004; Pyle et Spear, 2003; Gasser et al., 2015; Gervais et Crowley, 2017). Il est alors possible de lier indirectement ces réactions métamorphiques à des structures pour déterminer l'âge de déformation. Une méthode plus directe consiste à dater des minéraux néoformés dans des structures spécifiques. Dans le meilleur des cas, les grains datés forment eux-mêmes les indicateurs cinématiques (Williams et Jercinovic, 2012) ou certains groupes de monazite/zircon se trouvent principalement dans des types de structures spécifiques comme des ombres de pression (p. ex. Gervais et Brown, 2011) ou différentes générations de foliation (Williams et Jercinovic, 2012). Bien que ces méthodes soient robustes, elles requièrent de trouver ces structures clés au retour de la campagne de terrain. Une stratégie plus traditionnelle, mais avec une plus grande chance de réussite puisque développée directement sur le terrain, consiste à contraindre l'âge de déformation en datant différentes phases de matériel leucogranitiques (leucosome, dykes, sill) présentant des relations de recoupement sur le terrain (p. ex. Crowley et al., 2003; Gibson et al., 2005). 
En principe, cette méthode est robuste, mais plusieurs problèmes pratiques doivent être pris en compte pour que les résultats soient significatifs. Un problème évident concerne la présence de différentes générations de surcroissance sur les minéraux datés (zircon et monazite), et la possibilité de noyaux hérités dans le liquide. La méthode commune de dilution isotopique au spectromètre de masse par ionisation thermique (ID-TIMS) peut être inappropriée lorsque ces minéraux sont intégralement dissous, ce qui donne alors des résultats discordants (Foster et al., 2004; Gibson et al., 2005). Un exemple probant de ce problème provient de la Cordillère canadienne où l'interprétation d'une déformation précambrienne dans le cœur du dôme Frenchman Cap du Complexe de Monashee, basée sur des âges discordants obtenus par ID-TIMS sur les granites de la Suite de Bourne (Crowley, 1999), a été remise en question par les âges éocènes obtenus par la méthode SHRIMP (sonde ionique de très haute résolution spatiale) dans le cœur du dôme Thor-Odin plus au sud (Vanderhaeghe et al., 1999). Ce n'est qu'en redatant les bordures de zircon des dykes de granite par une autre méthode à haute résolution spatiale (ablation laser) et en combinant ces données à des relations de recoupements systématiques, et à la composition chimique du zircon et de la monazite que l'interprétation initiale, d'une déformation précambrienne (Crowley, 1999), a pu être confirmée (Crowley et al., 2008; Gervais et al., 2010). La datation par ablation laser, de par sa précision de 1-3\%, fonctionne bien pour différencier deux évènements tectoniques proches dans les orogènes jeunes comme la Cordillère canadienne ou l'Himalaya, ou deux évènements éloignés dans les orogènes précambriens, mais dans ces derniers, la précision est en limite d'application pour des évènements tectoniques proches. Par exemple, Soucy La Roche et al. (2015) ont clairement documenté la présence de quatre groupes compositionnels de zircon dans une pegmatite grenvillienne (ca. $1060 \mathrm{Ma}$ ), mais il était impossible de les distinguer par la méthode d'ablation laser utilisée, qui retourne des 
incertitudes de 15-20 millions d'années. La stratégie combinant les deux types d'analyses présentée dans notre étude palie à ces problèmes.

La Province de Grenville s'est formée lors d'une évolution tectonique complexe ayant culminée entre 1090 et 980 Ma par l'orogénie grenvillienne, et se divise en deux ensembles, la Ceinture parautochtone au NO, et la Ceinture allochtone au SE (Rivers et al., 1989; Rivers, 1997, 2008; Corrigan et al., 2000; Gower et Krogh, 2002; Rivers et al., 2012). Le premier, constitué de roches $\mathrm{du}$ socle du craton nord-américain et de sa couverture méta-sédimentaire, soit de roches provenant du cœur de la Laurentia (Fig. 1A; Carr et al., 2000; Rivers et Corrigan, 2000; Gower et Krogh, 2002; Rivers, 2008; Hynes et Rivers, 2010; Rivers et al., 2012), a été déformé et métamorphisé aux faciès schiste vert à granulite, respectivement à proximité du Front de Grenville et plus au SE, lors de la phase Rigolet de l'orogénie grenvillienne, soit entre 1000 et 980 Ma (Rivers, 2008). Le second, constitué de roches majoritairement péri-cratoniques à exotiques, soit localisées à la marge ou au large de la Laurentia, chevauchées sur le craton nordaméricain (Fig. 1A; Carr et al., 2000; Rivers et Corrigan, 2000; Gower et Krogh, 2002; Rivers, 2008; Hynes et Rivers, 2010; Rivers et al., 2012), a été métamorphisé aux faciès amphibolite supérieur à granulite pendant la phase Ottawan de l'orogénie grenvillienne, soit entre 1090 et 1040-1020 Ma (Fig. 1A; Rivers et al., 1989, 2012).

La zone de charriage basale de la Ceinture allochtone (Allochthon Boundary Thrust ou ABT), est une zone de cisaillement inverse d'échelle crustale, à vergence NO, présente dans l'ensemble de la province (Fig.1A). Cette structure, aurait amené le chevauchement de la Ceinture allochtone sur la Ceinture parautochtone lors de l'Ottawan (Rivers, 2008). Subséquemment, à la fin de l'Ottawan et lors du Rigolet, l'ABT serait devenue inactive, et la propagation du front de compression vers 
l'avant-pays, aurait activé le Front de Grenville qui correspond à chevauchement de la Ceinture parautochtone sur l'avant-pays (Rivers, 2008).

Malgré qu'une déformation ductile et pénétrative soit observée sur toute la longueur de la Ceinture parautochtone et qu'elle soit généralement parallèle au Front de Grenville, l'âge de cette déformation varie de grenvillien à archéen (Rivers, 1983a; Owen et al., 1986, 1988; Berclaz et al., 1995; Bethune, 1997; Krogh et al., 2002; Martignole et Martelat, 2005; van Gool et al., 2008). Dans la Province de Grenville orientale (Labrador) et dans la partie ouest de la Province de Grenville centrale (Québec, Chibougamau), des relations de recoupement avec les gabbros mésoprotérozoïques (Rivers, 1983a; Schärer et al., 1986; Owen et al., 1986, 1988; Brown et al., 1992; Connelly et Heaman, 1993; Daigneault et Allard, 1994; van Gool et al., 2008) et par une pegmatite syn-déformation de 1730 Ma (âge discordant dans Krogh et al., 2002) démontrent la présence d'une gneissosité pré-grenvillienne. Cette déformation secondaire n'est préservée qu'à proximité du Front de Grenville où le métamorphisme grenvillien est au faciès schiste vert alors qu'elle est oblitérée par la déformation principale grenvillienne, à des faciès métamorphiques plus élevés au SE, soit vers le cœur de l'orogène (Rivers, 1983b; van Gool et al., 2008).

Par contraste, dans la Province de Grenville occidentale en Ontario, les dykes de Sudbury cristallisés à $1235 \mathrm{Ma}$ et plissés par l'orogénie grenvillienne, recoupent une fabrique gneissique principale. Bien qu'elle soit généralement parallèle aux fabriques grenvilliennes, cette gneissosité principale est nécessairement anté-grenvillienne, et a été reliée à un évènement tectonique d'origine méconnue fin Pinwarien, à 1450 Ma (Bethune, 1997). À l'ouest du Québec (est de la route 117), des dykes mafiques paléoprotérozoïques à mésoprotérozoïques recoupent localement la fabrique gneissique principale (Martignole et Martelat, 2005), ce qui supporte l'interprétation de Berclaz et al. (1995) voulant que cette fabrique soit archéenne. 
Somme toute, dans la Province de Grenville occidentale, la préservation d'une fabrique prégrenvillienne pénétrative parallèle au grain structural typiquement grenvillien (NE-SO) semble paradoxale puisque le métamorphisme grenvillien de haut grade aurait dû favoriser son oblitération complète, tel qu'observée dans la Province de Grenville orientale. Plusieurs hypothèses pourraient être proposées telle qu'une rhéologie des roches variable à l'intérieur de la Ceinture parautochtone, entraînant une réponse à la déformation différente ou encore des erreurs de localisation du Front de Grenville. Il est également possible que ce paradoxe ne soit qu'apparent et s'explique par des méthodes de datations inappropriées dans les terrains de haut grade métamorphique précambrien, pour les raisons abordées précédemment.

Nous présentons les résultats d'une étude basée sur des travaux de terrain effectués au sud du réservoir Manicouagan (Province de Grenville centrale), dans la partie sommitale de la Ceinture parautochtone près de l'ABT. L'analyse structurale présentée décrit les différentes phases de déformation cartographiées, puis l'analyse métamorphique établie la chronologie relative de ces phases avec les réactions métamorphiques. Dans le but d'obtenir les âges précis de ces phases de déformation, nous appliquons ensuite une nouvelle stratégie de datation géochronologique sur des échantillons d'intrusion et de leucosomes syn-déformations. Cette stratégie allie la haute précision spatiale de l'ablation laser avec un spectromètre de masse à plasma couplé par induction (LA-ICP-MS), requise pour distinguer les variations d'âge et de composition en éléments de terres rares (ÉTR) intra-granulaires de zircon pour tracer leur origine, à la haute précision de l'érosion chimique par dilution isotopique au spectromètre de masse par ionisation thermique (CA-ID-TIMS), nécessaire pour discriminer les âges des différentes générations de déformation. Ainsi, cette stratégie nous permet non seulement de distinguer les fabriques et le métamorphisme pré-grenvilliens et grenvilliens de cette région, mais aussi d'en déterminer l'âge exact. 


\section{Contexte géologique}

\section{Régions voisines au réservoir Manicouagan}

Dans les régions voisines au réservoir Manicouagan, des fabriques anté-grenvilliennes sont partiellement préservées. Dans la Province de Grenville orientale (Labrador ouest) près du Front de Grenville, une déformation archéenne granulitique dans les gneiss archéens contiguës à la Province du Supérieur a été documentée (Rivers, 1983a, 1983b; Brown et al., 1991; van Gool et al., 2008), alors qu'à proximité de l'Orogène du Nouveau Québec, les sédiments du Groupe de Knob Lake (Rivers, 1980) et les gneiss archéens ont été déformés lors de cette orogénèse ( 1820 Ma; Fig. 1A; Rivers, 1983a; Brown et al., 1992).

À ces endroits, les phases de déformation anté-grenvilliennes se distinguent des déformations grenvilliennes car les fabriques archéennes sont définies par un rubanement gneissique granulitique alors que les fabriques grenvilliennes sont définies par des assemblages minéralogiques de plus bas grade métamorphique (Rivers, 1983a; van Gool et al., 2008), ou parce que les fabriques anté-grenvilliennes sont recoupées par des gabbros mésoprotérozoïques, connus en tant que Suite intrusive de Shabogamo (Rivers, 1983a; Brown et al., 1992; van Gool et al., 2008). Ces gabbros se sont mis en place sous forme de filon-couches dans la stratigraphie préalablement plissée des roches méta-sédimentaires paléoprotérozoïques du Groupe de Knob Lake (Rivers, 1983a), ou sous forme de dykes qui recoupent la stratification et la foliation S1 dans ces méta-sédiments (Brown et al., 1992). Vers le sud de la Ceinture parautochtone, proche de l'ABT, la Suite intrusive de Shabogamo a donné par ID-TIMS deux âges U/Pb discordants d'interceptes supérieurs, et donc incertains, de $1452+15 /-13$ Ma sur baddeleyite et de $1459+23 /-$ 22 Ma sur zircon (Connelly et Heaman, 1993; Connelly et al., 1995). 
Plusieurs phases de déformation grenvilliennes ont été rapportées dans la Ceinture parautochtone. Dans cette étude, elles sont notées $\mathrm{D}^{*}$ ou $\mathrm{D}^{\prime}$ de façon à les distinguer des déformations observées dans la région étude. Dans la Province de Grenville orientale (Labrador ouest) à proximité du Front de Grenville, trois phases de déformation ont été décrites; soient : 1) une foliation S1* pénétrative à vergence $\mathrm{NO}$ et des plis isoclinaux $\mathrm{P} 1 * ; 2)$ des plis $\mathrm{P} 2 *$ et des chevauchements à vergence NO déformant la $\mathrm{S} 1 *$; puis 3 ) des plis $\mathrm{P} 3 *$ plurikilométriques plongeant vers le SE (Rivers, 1983a; van Gool et al., 2008). Dans la Ceinture parautochtone de la Province de Grenville centrale, à proximité de l'ABT, une phase de déformation D1' prédomine et devient pénétrative avec l'apparition de linéations parallèles aux axes de plis $\mathrm{P} 1$ '. Ces linéations et axes de plis plongent soit vers le SE, soit vers le NO, sous l'influence de plis P2' ouverts et NE-SO (Clarke, 1977). Enfin, l'absence de cartographie en continue de la Province de Grenville orientale à celle centrale ne permet pas d'établir les corrélations entre les phases de déformation de ces deux régions.

\section{Région à l'étude}

Le terrain d'étude se situe au SO du réservoir Manicouagan dans la Province de Grenville centrale (Fig. 1) et a fait l'objet de travaux de cartographie réalisés par le ministère de l'Énergie et des Ressources naturelles du Québec (MERNQ; Moukhsil et al., 2013). La Ceinture parautochtone, aussi appelée Terrane de Gagnon dans cette région, est formée du Groupe de Gagnon (Clarke, 1977) et du Complexe de Ulamen (Moukhsil et al., 2013). Ce complexe regroupe les roches du socle archéen, comprenant des gneiss tonalitiques datés à $2681 \mathrm{Ma}$ (datation U/Pb sur zircon par LA-ICP-MS et ID-TIMS; Davis et Dion, 2012a et b; Moukhsil et al., 2013), des gneiss granitiques et des gabbros gneissiques en interlits ou en dykes non datés (Fig. 1B). Le Groupe de Gagnon regroupe les roches de la couverture paléoprotérozoïque 
comprenant des paragneiss migmatiques, des formations de fer, des quartzites et des marbres. Bien que ces formations appartiennent vraisemblablement au Groupe de Knob Lake qui s'est déposé sur la marge de la Laurentia à 1900 Ma dans l'Orogène du Nouveau Québec (Rivers, 1980), leur nature hautement déformée et l'absence de certaines unités lithologiques dans la région d'étude, tel que les formations de Seward et d'Attikamagen, ne permettent pas d'établir une corrélation stratigraphique robuste. Ainsi nous préférons employer le terme Groupe de Gagnon dans cette étude pour assurer une continuité avec la terminologie établie lors de la cartographie récente de la région d'étude par Moukhsil et al. (2013). Les aluminosilicates présents dans les paragneiss sont la kyanite au NO de la région d'étude et la sillimanite au SE. Les paragneiss à kyanite ont suivi une boucle Pression-Température $(\mathrm{P}-\mathrm{T})$ serrée et de sens horaire, culminant à des pressions de $1250-1300 \mathrm{MPa}$ et à des températures de $815-830^{\circ} \mathrm{C}$ (Jordan et al., 2006; Indares et al., 2008). Pour les paragneiss à sillimanite, seul le chemin P-T rétrograde est connu et il a des conditions métamorphiques maximales de $1000 \mathrm{MPa}$ et de $875^{\circ} \mathrm{C}$ (Jordan et al., 2006; Indares et al., 2008). L'âge de cristallisation des leucosomes dans les champs de stabilité de la kyanite et de la sillimanite a été contraint à 995-985 Ma par datation U/Pb sur monazite, alors que l'âge de refroidissement sous des températures de 700-600 ${ }^{\circ} \mathrm{C}$ a été contraint à 961-956 Ma par datation $\mathrm{U} / \mathrm{Pb}$ sur titanite (Jordan et al., 2006).

$\mathrm{Au}$ SO du réservoir Manicouagan, les zones de cisaillement majeures sont l'ABT et la Zone de cisaillement Relay (Fig. 1B). Cette dernière était originellement interprétée comme le site de l'ABT (Hynes et al., 2000), mais les lithologies (Dunning et Indares, 2010; Moukhsil et al., 2013) et les âges modèles $\mathrm{Sm} / \mathrm{Nd}$ (Thomson et al., 2011), indiquent que la Zone de cisaillement Relay est localisée au cœur des gneiss archéens, bien que Dunning et Indares (2010) la considère comme un contact tectonique important marquant l'apparition d'abondants dykes de gabbro et de 
granite. Le tracé de l'ABT a donc été placé plus à l'est et définit le contour d'un antiforme plongeant vers le SE, dont les flancs sont orientés NO-SE, la charnière NE-SO et le cœur constitué de la Ceinture parautochtone (Fig. 1B).

Quelques données sont disponibles concernant l'âge de la déformation et du métamorphisme dans la Ceinture parautochtone de la région d'étude. Dans le Groupe de Gagnon, deux échantillons datés par $\mathrm{U} / \mathrm{Pb}$ sur monazite donnent une analyse concordante à $1738 \mathrm{Ma}$, et trois analyses discordantes formant une régression linéaire ayant un intercepte supérieur à 1719 Ma, interprétées comme un évènement métamorphique ancien (Jordan et al., 2006). Par contre, tous les âges U/Pb obtenus par la méthode LA-ICP-MS sur zircon dans les roches méta-sédimentaires sont discordants et forment une ligne de mélange avec des interceptes supérieur et inférieur respectivement de 1875 et de $\sim 1009$ Ma (Davis et Dion, 2012a). Les grains de zircon analysés dans le Complexe de Ulamen, ne semblent pas avoir enregistrés cet évènement de $1738 \mathrm{Ma}$ puisqu'aucune analyse concordante n'a retourné un âge compris entre 2442 et 1016 Ma et que les analyses discordantes forment une ligne de mélange avec des interceptes archéens et grenvilliens (Jordan et al., 2006; Davis et Dion, 2012a et b). Cependant, la présence d'une déformation archéenne a été rapportée par Dunning et Indares (2010) qui ont documentés un dyke de granite recoupant la foliation dans les gneiss archéens. L'âge d'intrusion de ce dyke est estimé à $1741 \pm 32$ Ma par l'intercepte supérieur d'une régression linéaire passant par quatre analyses discordantes (Dunning et Indares, 2010). Ainsi, les données disponibles sur l'existence d'un évènement tectono-métamorphique protérozoïque anté-grenvillien dans la région d'étude sont ambigües.

\section{Description litho-tectonique}

Les affleurements de roches du Complexe de Ulamen contiennent des orthogneiss tonalitiques à granitiques voir charnockitiques rubanés, généralement interlités avec des niveaux mafiques de 
composition gabbroïque à gabbronoritique, voir amphibolitique puisque les pyroxènes ne sont plus distinguables. Cependant, certains affleurements ne sont composés que de roches mafiques homogènes et massives. Les interlits aussi bien felsiques que mafiques sont d'épaisseur infracentimétrique à pluri-métrique et peuvent être boudinés. Ces orthogneiss felsiques et mafiques sont généralement migmatitiques avec un pourcentage de leucosome très variable de moins de $5 \%$ à plus de $70 \%$, avec une moyenne de $15-35 \%$ pour les orthogneiss felsiques et une moyenne de 5 à $20 \%$ pour les orthogneiss mafiques.

Les affleurements de roches du Groupe de Gagnon contiennent généralement des paragneiss migmatiques, et ils contiennent localement des niveaux de quartzite interlités avec de marbres et des niveaux de roches calcosilicatées interlités avec des niveaux siliceux interprétés comme des métacherts. Le pourcentage de leucosome varie de 5 à $90 \%$ avec une moyenne 15 à $40 \%$ dans les paragneiss. Enfin, des paragneiss du Groupe de Gagnon peuvent être observés en lambeaux dans les orthogneiss, des boudins de roches ultramafiques peuvent être continus dans les orthogneiss et dans les paragneiss, et localement des dykes de gabbro intrudent les orthogneiss, notamment dans la Zone de cisaillement Relay.

Dans les orthogneiss du Complexe de Ulamen et les paragneiss du Groupe de Gagnon, la foliation est généralement définie par les interlits et les orientations préférentielles des minéraux et des leucosomes. Localement dans les paragneiss, une distinction entre les différentes phases de déformation peut être effectuée puisque qu'une première génération de leucosome définie une première foliation et qu'une deuxième génération de leucosome définie une deuxième foliation. La linéation est définie par l'orientation préférentielle des grains d'amphibole contenus dans les orthogneiss felsiques et mafiques ou dans les dykes de gabbro recoupant la foliation. Localement elle est également marquée par l'étirement des grains de quartz dans les paragneiss et dans les 
orthogneiss felsiques, et localement par l'orientation préférentielle de la sillimanite dans les paragneiss. Enfin, le changement de conditions métamorphiques au faciès granulite, marqué dans les paragneiss par la présence de kyanite au NO de la région d'étude et par la présence de sillimanite au SE de cette région, préalablement documenté par Jordan et al. (2006) et Indares et al. (2008), a également été observé. Ce changement s'accompagne d'une transition dans le grain structural et pourrait indiquer un niveau structural inférieur à l'ouest de la région d'étude, et supérieur à l'est de cette région.

\section{Géologie structurale}

\section{Méthodologie et nomenclature}

Cette section présente une analyse structurale de la Ceinture parautochtone, dans la région d'étude, réalisée à partir nos données de terrain et de celles prisent lors de la cartographie du MERNQ (Moukhsil et al., 2013). Les linéations mesurées sont des linéations minérales ou d'étirement. Les cisaillements sont notés décrochant-inverse ou décrochant-normal pour un angle de chute d'une linéation inférieur à $45^{\circ}$, et inverse-décrochant ou normal-décrochant pour un angle supérieur à $45^{\circ}$. Les sens de cisaillement ont été déduis de divers indicateurs cinématiques, généralement au nombre de 2 à 5 par affleurement, observés dans le plan XZ de l'ellipsoïde de déformation. Les intensités des déformations ont été évaluées qualitativement sur une échelle de 1 à 5 (5 correspondant à une déformation très intense, figure $1 \mathrm{~B})$ et les critères de cette évaluation sont décrits dans l'appendice 1. La zone d'étude a été divisée en six secteurs selon différents critères, soient: 1) les attitudes des fabriques (Fig. 2); 2) l'intensité de la déformation; 3) l'évidence de zones de cisaillement; et 4) l'évidence de phases de déformation distinctes. Ces fabriques ont été mesurées aussi bien dans le Groupe de Gagnon paléoprotérozoïque que dans les 
gneiss archéens du Complexe de Ulamen que nous n'avons pas pu départager sur la base des structures.

La carte géologique détaillée du secteur B (Fig. 3) a été réalisée à partir des données du MERNQ et des levés magnétiques (D'Amours et Intissar, 2012a, 2012b) auxquels ont été appliqués les filtres de dérivée verticale d'ordre 1 et de tilt (Geosoft, 2007). La présence des formations de fer a été supposée dans le cas d'une anomalie fortement positive visible sur la carte du champ magnétique résiduel $(550,9$ à $14948,7 \mathrm{nT})$ et sur la carte du gradient magnétique $(0,99$ à 81,89 nT/m). Sur la carte du gradient magnétique, ces anomalies positives sont communément entourées d'une anomalie fortement négative, comprise entre -0,76 à -29,84 nT/m. Les contours géologiques ont été tracés en s'appuyant sur les foliations mesurées et sur les lignes d'anomalies positives et négatives du gradient vertical magnétique et du tilt. Les traces des structures interprétées sont d'ailleurs une combinaison de ces lignes et des foliations observées sur les affleurements; la quantité de ces traces a ensuite été augmentée afin de faire ressortir les plis.

Finalement, dans la Ceinture parautochtone, trois grandes phases de déformation ont pu être observées, soit D1, D2 et D3. Les fabriques et les cisaillements sont présentés par secteur et sont suivis d'une description des indices permettant d'établir l'ordre chronologique relatif des phases de déformations. Ces fabriques n'impliquent pas nécessairement des phases de déformations régionales distinctes et peuvent être le produit de la déformation progressive (Williams, 1985). Il est aussi possible qu'une phase D2 à un affleurement donné corresponde à une D1 d'un autre, et ce problème sera discuté dans la discussion. 


\section{Résultats}

\section{Description des structures}

\section{$\underline{\text { Secteurs A à C }}$}

Les secteurs A à $\mathrm{C}$, localisés au NO de la région à l'étude (Fig. 1B), présentent deux groupes de déformation définis par des maximas de fabriques planaires et de fabriques linéaires différents. Dans le secteur A, dont les données ne proviennent que du MERNQ, le pôle de foliation du premier groupe indique une foliation orientée ONO-ESE avec un fort pendage vers le SSO, tandis que le pôle du second groupe indique une foliation globalement orientée NE-SO avec un pendage modéré vers le SE (tableau 1, Fig. 2, 4). Les linéations forment un maxima à plongement modéré vers le SSE, probablement associées au premier groupe de foliation, puisqu'au NO de la région d'étude, la seconde déformation est peu pénétrative. Ce point sera discuté subséquemment.

Dans le secteur B, (tableau 1, Fig. 2, 4) la foliation est généralement orientée NE-SO à pendage modéré à fort vers le $\mathrm{SE}$, et semble équivalente au deuxième groupe du secteur $\mathrm{A}$, mais localement la foliation est ESE-ONO à pendage modéré à fort et semble équivalente au premier groupe du secteur A. D'après les mesures du MERNQ, deux pôles de linéation toutes deux obliques sont également visibles sur le stéréogramme, celui appartenant au premier groupe est $38 \rightarrow$ N138 et celui appartenant au deuxième groupe est $28 \rightarrow$ N201. Sur l'affleurement 12SJ14 exposant des paragneiss du Groupe de Gagnon, une foliation N101/68 définie par une première génération de leucosome est recoupée par une schistosité de plan axial qui correspond au deuxième groupe et est définie par une deuxième génération de leucosome (Fig. 5A1). Un mouvement apparent senestre pluri-centimétrique est visible le long de ces plans axiaux NE-SO à fort pendage (Fig. 5A). Les axes de plis portés par cette schistosité dans le secteur B, ont des attitudes très variables, soit fortement plongeant vers le NE et le SE, soit à plongement modéré 
vers le SO (Fig. 2). Sur la carte géologique et le schéma structural, deux traces de plan axial, de plis isoclinaux NO-SE et de plis ouverts NE-SO, indiquent la présence de plis superposés appartenant respectivement au premier et au deuxième groupes (Fig. 1B et 3). Le Groupe de Gagnon qui constitue un niveau repère, puisqu'il correspond à des sédiments protérozoïques déposés sur le craton archéen, dessine également des motifs en croissant typiques de plis superposés (Fig. 3; type 2 de Ramsay, 1967). D'après l'interprétation du levé magnétique, à l'ouest et à l'est de la carte (Fig. 3), les gneiss du socle sont respectivement en contact avec une formation de fer et avec des marbres incluant des formations de fer. Ces relations suggèrent une gradation stratigraphique latérale qui est peu probable sur les quelques kilomètres séparant ces deux contacts, bien que ceux-ci soient plissés. Une hypothèse pouvant expliquer ces deux contacts serait la présence d'un chevauchement cryptique anté-D1 qui les aurait juxtaposés.

Dans le secteur $\mathrm{C}$, les foliations ont généralement une attitude NO-SE avec un pendage modéré vers le NE, identique au premier groupe du secteur A, mais certaines sont NE-SO à pendage modéré à fort vers le SE, et semblent identiques au deuxième groupe du secteur A (tableau 1, Fig. 2, 4). Les linéations ne sont pas groupées, mais plongent en général modérément vers les quadrants NE et SE. Selon les données du MERNQ (Moukhsil et al., 2013), les linéations obliques plongeant modérément vers le SE et portées par les foliations NO-SE impliquent des cisaillements senestres et dextres. Sur ces affleurements, les axes de plis à plongement modéré vers le SE sont parallèles aux linéations. Ainsi, dans les secteurs A à $\mathrm{C}$, deux populations de déformation sont visibles. L'une est une foliation ONO-ESE portant une linéation SE et impliquant un mouvement du bloc SSO vers le NO, tandis que l'autre est une schistosité de plan axial NE-SO à mouvement senestre. 


\section{$\underline{\text { Secteur D }}$}

Dans le secteur D, localisé au NE de la région d'étude (Fig. 1B), la foliation a une orientation qui varie de NNO-SSE à E-O et un pendage vers le SE allant de 20 à $90^{\circ}$, avec un pic de densité N029/65 (tableau 1, Fig. 2, 4). Sur les rives du réservoir Manicouagan, les pôles des foliations se répartissent globalement sur un plan moyen N280/75, indiquant que cette fabrique est plissée. L'orientation de l'axe de pli correspondant au pôle de ce plan, soit $15 \rightarrow \mathrm{N} 190$, concorde avec les mesures des axes de plis relevées sur le terrain (Fig. 2). Ces plis sont communément isoclinaux et plongent généralement vers le SSO, bien que quelques mesures donnent un plongement vers le NE. La linéation $21 \rightarrow \mathrm{N} 189$ est parallèle aux axes de plis plongeant vers le SSO (tableau 1, Fig. 2) et elle est marquée par l'orientation des amphiboles dans les orthogneiss archéens concordant à la foliation et dans le dyke de gabbro recoupant la S2 observé dans la Zone de cisaillement Relay, ainsi que par l'étirement des minéraux, dont les grains de quartz dans certains échantillons (Fig. 5D1). Les indicateurs cinématiques observés dans ce secteur montrent un mouvement du bloc SE vers le NNE, tel qu'indiqué par des fabriques $\mathrm{C} / \mathrm{S}$ et des porphyroclastes de type $\delta$ ou $\sigma$ (Fig. $5 \mathrm{C}$, 5D2 et 5E1). Puisque la foliation est plissée selon un axe parallèle à la linéation, le cisaillement observé à l'affleurement est senestre-inverse sur les flancs NO du pli, inverse au niveau de la charnière et dextre-normal sur les flancs SE. Enfin, au cœur de la zone de cisaillement Relay, les attitudes des fabriques planaires et linéaires ainsi que le cisaillement senestre observé (Fig. 5E), similaires à ceux de la phase de déformation D2 indiquent que cette zone de cisaillement est une structure D2.

\section{$\underline{\text { Secteur E }}$}

Les fabriques, dans le secteur E, ont une orientation différente de celles du secteur D (tableau 1, Fig. 1B, 2 et 4). En effet, la foliation, n'est pas plissée, mais elle est orientée ENE-OSO; la 
linéation, quant à elle, plonge vers le sud et est oblique (tableau 1, Fig. 2). La différence la plus notable entre ces deux secteurs est la transposition par cisaillement, dans le secteur E, de la linéation sud par une phase de déformation localisée parfois très pénétrative (Fig. 5G1) à laquelle est associée une linéation OSO portée par une foliation toujours ENE-OSO (tableau 1, Fig. 2). La distinction entre ces deux linéations a pu être effectuée à l'affleurement quand la linéation sud est déformée par des cisaillements locaux.

\section{$\underline{\text { Secteur F }}$}

Dans le secteur F, la foliation orientée ONO-ESE avec un fort pendage est combinée à une linéation plongeante vers le SE (tableau 1, Fig. 2, 4). Le cisaillement est dextre et inverse-dextre tandis que les axes de plis $30 \rightarrow \mathrm{N} 111$ sont parallèles aux linéations (tableau 1, Fig. 1B et 2).

\section{$\underline{\text { Résumé }}$}

En résumé, deux déformations distinctes peuvent être définies, d'après les attitudes de leurs fabriques différentes (Fig. 4) et leurs expressions par deux générations de leucosome respectives. La première, présente dans les secteurs occidentaux (A-C et F), est définie par une foliation transverse à l'orogène, ONO-ESE portant une linéation oblique SE, tandis que la deuxième, présente dans les secteurs orientaux (B-E), est définie par une foliation parallèle à l'orogène, NE$\mathrm{SO}$, associée à une linéation oblique SSO. En outre, les cisaillements sont inverses à décrochants pour les deux déformations, mais pour celle présente dans l'ouest le transport tectonique s'est effectué vers le NO, tandis que pour l'autre déformation le transport tectonique s'est effectué vers le NNE. Finalement les axes des plis isoclinaux de la première déformation plongent vers le SE, et semblent similaires à l'antiforme à cœur parautochtone et à plongement $\mathrm{SE}$, marqué par le tracé de l'ABT (Fig. 1B), mais les éléments de cette étude ne sont pas suffisants pour déterminer si l'ABT a été plissée par la déformation D1 ou par une phase de déformation postérieure. 


\section{Superposition de la déformation D2 sur la déformation D1}

La superposition des deux déformations décrites précédemment, est renseignée par des indices provenant du secteur B. Les traces des plis sur le schéma structural et la carte géologique, montrent que les plis NO-SE sont affectés par les plis NE-SO (Fig. 1B et 3) et que les unités lithologiques forment des patrons en croissant. L'affleurement 12SJ14 est clé puisqu'il est localisé à la charnière d'un pli P2 (Fig. 3). Sur cet affleurement, la présence d'une schistosité de plan axial NE-SO plissant et cisaillant par un mouvement senestre une foliation N101/68 (Fig. 5A) confirme que les deux populations de foliation ONO-ESE et NE-SO du secteur A (Fig. 2, 4) sont très probablement, respectivement, la $\mathrm{S} 1$ et la $\mathrm{S} 2$. En outre, leurs expressions minéralogiques, présentées dans la section suivante portant sur les contraintes métamorphiques aux phases de déformation, sont différentes et permettent de distinguer ces deux phases de déformation. Combiné au patron de la carte de la figure 3, ces relations nous amènent à interpréter les fabriques planaires ONO-ESE, localisées dans les secteurs occidentaux (A, B, C et F), comme étant antérieures aux fabriques planaires NE-SO localisées dans les secteurs orientaux (B à E).

Dans le secteur A, une seule linéation plongeant vers le SE est présente, nous assumons donc qu'elle est liée à la déformation majeure du secteur et serait une L1 (Fig. 2, 4). Au contraire, dans le secteur B, le pôle de la S1 sur le stéréogramme (Fig. 2) est moins marqué puisque les plis P2 sont serrés. Les deux populations de linéation L1 et L2, plongeant respectivement vers le SE et le SSO sont présentes dans ce secteur (Fig. 2, 4). Les axes de plis P2 dans ce secteur B ne sont pas parallèles à la linéation L2. Dans les secteurs D et E, la foliation S1 est parallèle à la S2, orientée NE-SO, sur les flancs des plis P2 (Fig. 2, 4). La linéation L1 est oblitérée et seules les linéations L2 plongeantes vers le SSO et parallèles aux axes de plis P2 sont encore visibles. Les indicateurs cinématiques témoignent d'un mouvement décrochant-inverse du bloc SE vers le NNE lors de la 
déformation D2 (Fig. 5). Les linéations OSO décrites dans le secteur E, sont issues d'une déformation plus tardive, la D3 (Fig. 4). Dans le secteur F, les fabriques sont identiques à celles issues de la déformation $\mathrm{D} 1$ décrite dans les secteurs $\mathrm{A}$ à $\mathrm{C}$, soit une foliation ONO-ESE et une linéation SE. D'après les données du MERNQ, les cisaillements associés à ces deux fabriques indiquent un mouvement dextre-inverse du bloc SSO vers le NO (tableau 1, Fig. 2, 4). Ces caractéristiques laissent penser que dans ce secteur seule la déformation D1 est présente. Ainsi, la déformation D1 est essentiellement présente dans les secteurs à l'ouest de la région d'étude, soient les secteurs $\mathrm{A}$ à $\mathrm{C}$ et $\mathrm{F}$ (Fig. 1B, 2 et 4), tandis que la déformation D2 est plus marquée dans les secteurs à l'est de la région à l'étude, soit les secteurs D et E. Le corollaire de cette conclusion est que le transport tectonique s'est effectué respectivement vers le NO sur un plan perpendiculaire à l'orogène et vers le NNE sur un plan parallèle à l'orogène lors des phases de déformation D1 et D2.

\section{Chronologie relative de l'anatexie et des déformations D1 et D2}

Dans l'ensemble de la région à l'étude les paragneiss sont migmatitiques et contiennent des leucosomes (selon la définition non génétique de Mehnert, 1968). La genèse de ces leucosomes, étroitement liée aux déformations D1 et D2, a pu être retracée sur des affleurements capitaux comme celui 12SJ14 (Fig. 1B). Ces leucosomes de composition granitique, localement à texture pegmatitique, sont interconnectés, espacés de $5-15 \mathrm{~cm}$ et ont une épaisseur variant de 2 à $15 \mathrm{~cm}$ (Fig. 5A2). Ils contiennent également des grains de quartz non déformés et localement des grains de grenat automorphes, et ils sont très souvent entourés de mélanosomes riches en biotite. Bien que l'hypothèse de migmatites d'injection ne puisse être écartée, la présence de mélanosomes, la présence locale de grenat péritectiques et l'absence de déformation dans les leucosomes est interprétée comme une fusion partielle in-situ des migmatites. Cette interprétation est supportée 
par les assemblages métamorphiques et les textures observées à l'échelle de la lame mince et décrits dans la section suivante. Certains leucosomes sont plissés et cisaillés par la déformation D2, d'autres, non déformés, ont cristallisé dans les plans de schistosité de plan axial P2, tandis qu'un rubanement migmatitique définit la foliation $\mathrm{S} 1$. Ces constatations suggèrent une cristallisation des leucosomes, issus de la fusion partielle des paragneiss, en partie synchrone à la déformation D1 et en partie pré- à syn-déformation D2.

\section{Transposition progressive des fabriques vers $l^{\prime} A B T$}

La transposition de D1 par D2 est progressive et s'accentue vers l'ABT. Cette progression est caractérisée, dans le secteur B, par une parallélisation incomplète de la S1 sur les flancs des plis $\mathrm{P} 2$ et d'une préservation de la $\mathrm{S} 1$ aux charnières où la $\mathrm{S} 2$ est une schistosité de plan axial (Fig. 3 et 5A). Plus à l'est, la transposition est quasiment complète. Dans le secteur D, la S1 est effectivement parallèle à la S2 sur les flancs des plis (Fig. 5B) et ces plis sont communément isoclinaux. Les axes de plis P2 sont caractérisés dans le secteur B, par un plongement fort de direction variable, qui, vers le SE, devient progressivement parallèle à la linéation L2 (Fig. 5D1). En effet, dans le secteur D, l'axe de pli calculé d'après les pôles de foliation a un plongement faible vers le SSO, identique aux axes de plis et linéations mesurés sur le terrain (tableau 1, Fig. 2, 4). Cette transformation d'une absence de parallélisme entre les axes de plis et les linéations, dans le secteur B, à un parallélisme dans le secteur D, confirme l'augmentation de la transposition vers l'ABT.

L'obliquité des linéations L1 et L2 pourrait indiquer un régime transpressif (Dewey et al., 1998; Tikoff et Fossen, 1999), qui pourrait être causé par une structuration du socle sous-jacent et ne refléterait pas nécessairement des contraintes régionales. Néanmoins, les linéations ne se 
répartissent pas sur un grand cercle tel que modélisé pour ce type de régime par Jiang (2014). Une analyse cinématique quantitative serait donc requise pour vérifier cette hypothèse.

L'intensité qualitative des déformations, documentée sur le terrain, corrobore cette transposition progressive avec une plus grande proportion d'intensité qualitative de 3 à 5 (cercles jaune à bordeaux sur la figure 1B) vers l'ABT et le SE. Dans les secteurs B et F, l'intensité des déformations est généralement faible à moyenne (Fig. 1B). Dans le secteur $\mathrm{D}$, l'intensité de la déformation D2 est variable; elle peut être faible à moyenne ou atteindre localement des valeurs aussi grandes que celles observées dans la Zone de cisaillement Relay (tableau 1, Fig. 1B et 5E2). Dans le secteur E, l'intensité de la déformation augmente vers le sud, donc vers l'ABT, et peut passer de moyenne à très intense. Ainsi l'intensité qualitative des déformations corrobore la transposition des axes de plis vers le SE de la région à l'étude et vers l'ABT. L'augmentation de la déformation $\mathrm{D} 2$ vers le SE et compatible avec la localisation de l'ABT, définie par la cartographie du MERNQ et les âges modèles Sm/Nd (Thomson et al., 2011; Moukhsil et al., 2013). La Zone de cisaillement Relay ne serait donc pas une zone de cisaillement unique délimitant les ceintures parautochtone et allochtone, tel que suggéré par Hynes et al. (2000), mais serait une structure D2 qui correspondrait à un couloir de déformation intense dans la Ceinture parautochtone.

\section{Phases de déformation décrochante-normale tardi-D2 et D3}

Une déformation, très localisée et observée dans le secteur D sur l'affleurement 12SJ27 (Fig. 5E3), consiste à un plan de cisaillement senestre-normal, NE-SO à fort pendage, recoupant la foliation S2. L'attitude et la composante principale de cisaillement sont identiques à la déformation D2, suggérant une même appartenance, mais puisque ce plan de cisaillement recoupe la foliation S2, sa phase de déformation a été notée tardi-D2. 
Une dernière phase de déformation localisée a été observée uniquement sur quelques affleurements du secteur E, exposant des paragneiss du Groupe de Gagnon et des gneiss archéens (Fig. 1B). Par contre, cette phase pourrait être plus commune qu'il n'y parait puisque les affleurements de ce secteur sont relativement rares et de mauvaise qualité. Sur l'affleurement de qualité $12 \mathrm{SJ} 47$, la linéation L2 $50 \rightarrow \mathrm{N} 180$ devient $10 \rightarrow \mathrm{N} 235$ lorsqu'elle est située sur un plan de foliation et déformée avec lui par un cisaillement tardif C3 dont le mouvement est opposé à celui de la déformation D2 (tableau 1, Fig. 2). Bien que sur cet affleurement, le cisaillement tardif est très localisé, sur l'affleurement 12SJ49, seule une linéation L3, parallèle à la linéation plissée par C3 de l'affleurement précédent et indiquant un mouvement opposé à celui de la déformation D2, peut être observée (Fig. 1B). La foliation y est orientée ENE-OSO, la déformation est intense, et un leucosome porphyroclastique de type $\delta$ indique que le cisaillement est dextre-normal (tableau 1, Fig. 1B et 5G). La présence de leucosome dans les plans de foliation S3 dont les quartz sont en ruban, et cet indicateur cinématique recoupant la foliation et cisaillé par la déformation D3, dénote tout deux d'une cristallisation de leucosomes, issus de la fusion partielle des paragneiss, pré- à syn-D3. Ainsi, D3 étant une phase de déformation localisée, aucune déformation normale majeure n'est présente dans la région à l'étude.

\section{Un âge protérozö̈que pour les trois phases de déformation}

Cette description structurale suggère dès lors que les déformations D1 à D3 ne peuvent être archéennes. En effet, dans toute la région d'étude, les mesures ont été prélevées aussi bien dans le Complexe de Ulamen archéen que dans les paragneiss paléoprotérozoïques du Groupe de Gagnon. D'après nos données, les foliations, les linéations et les cisaillements sont parallèles et identiques dans les deux unités, les trois déformations affectent donc de façon identique les roches archéennes et paléoprotérozoïques. Les reliques du litage compositionnel intrusif des 
gneiss archéens, et le contact du Groupe de Gagnon sur le Complexe de Ulamen sont parallèles aux foliations et oblitérés par les déformations D1 à D3, au point que leur origine sédimentaire, intrusive ou chevauchante n'est plus identifiable. Ces trois déformations sont donc pénétratives et postérieures aux dépôts du Groupe de Gagnon, et sont donc protérozoïques et non archéennes.

\section{Contraintes métamorphiques aux phases de déformation}

Les paragneiss et un gabbro permettent de relier les phases de déformations au métamorphisme. Tous les paragneiss, reliés aux déformations D1, D2 ou D3 sont migmatitiques et ont une texture inéquigranulaire et granolépidoblastique, avec une alternance de niveaux riches en biotite, \pm aluminosilicates et de niveaux riches en quartz, plagioclase et feldspath alcalin (Fig. 6 et 7). Ces aluminosilicates sont de la kyanite dans le secteur B et de la sillimanite dans les secteurs C et D. Ils contiennent également des porphyroblastes de grenat et ponctuellement de la muscovite (Fig. 6 et 7). Malgré ces similarités, les paragneiss contenant des aluminosilicates ont permis de relier, dans le secteur B, la kyanite prograde à la déformation D1 et dans les secteurs C et D, la sillimanite rétrograde à la déformation D2.

\section{Début d'anatexie syn-D1 dans les paragneiss à kyanite}

Dans le secteur B, les paragneiss contenant localement de la kyanite exclusivement prograde (affleurement 13SJ182) révèlent que la déformation D1 était supra-solidus (Fig. 1B et 6). Les grains de kyanite présents dans la matrice sont orientés parallèlement à la foliation S1 et ont localement une extinction roulante comme la plupart des grains de biotite, bien qu'une deuxième génération de biotite, sans orientation préférentielle soit localement présente (Fig. 6A, 6D, 6E et 6G). La déformation D1 était donc syn- à post-cristallisation de kyanite et biotite progrades. De la kyanite automorphe, de plus grande taille que celle présente dans la matrice, a cru dans des leucosomes (Fig. 6A). Ces textures indiquent une croissance de la kyanite en présence de liquide 
qui n'est possible que par la réaction de déshydratation-fusion de la muscovite (Spear et al., 1999)

$$
\mathrm{R} 1: \mathrm{Ms}+\mathrm{Qtz}+\mathrm{Pl} \rightarrow \mathrm{Kfs}+\mathrm{Al}_{2} \mathrm{SiO}_{5}+\mathrm{Liq} \text { (abréviations selon Kretz, 1983) }
$$

L'orientation subparallèle à S1 des leucosomes et de la kyanite dans ces leucosomes contenant des grains de quartz non déformés (Fig. 6A), démontre que la déformation D1 s'est déroulée en présence de fusion partielle. Dans la matrice, les grains de kyanite et ceux de biotite de première génération, tous orientés, ont donc cru lors du chemin prograde avant la réaction $\mathrm{R} 1$ et la déformation D1. Les intercroissances lobées de grenat et de quartz entourant la kyanite dans la matrice (Fig. 6B), suggèrent que le grenat est néoformé en présence de liquide de fusion partielle (Waters, 2001) et qu'il remplace la kyanite. Par conséquent, le grenat s'est formé par la réaction de déshydratation-fusion de la biotite dans le champ de la kyanite (Spear et al., 1999) :

$$
\mathrm{R} 2: \mathrm{Bt}+\mathrm{Al}_{2} \mathrm{SiO}_{5}+\mathrm{Qtz} \rightarrow \mathrm{Kfs}+\mathrm{Grt}+\mathrm{Liq} .
$$

Cette réaction est continue, mais possède des isoplètes quasiment isothermaux pour des compositions de métapélites (p. ex. Gervais et Crowley, 2017). La préservation de nombreux grains de biotite orientés suggère également que cette réaction R2 n'a pas été complète, et qu'elle se poursuivait lors du pic métamorphique. La biotite non orientée de seconde génération (Fig. 6D et $6 \mathrm{E}$ ) est vraisemblablement issue de la réaction rétrograde inverse, notée Re-R2, puisque les grains de grenat sont localement très légèrement corrodés par la biotite de seconde génération. La muscovite remplace aussi de façon incomplète la biotite (Fig. 6G); la muscovite est donc rétrograde et s'est probablement formée lors de la réaction rétrograde inverse à $\mathrm{R} 1$, notée Re-R1. Ainsi, avant d'atteindre le pic métamorphique, cet échantillon a été partiellement fondu dans le champ de la kyanite par déshydratation-fusion de la muscovite (R1), puis de la biotite (R2). 
Ensuite, il a suivi un chemin rétrograde qui a permis la cristallisation d'une deuxième génération de biotite et celle de la muscovite grâce aux réactions Re-R1 et Re-R2. L'absence de sillimanite et de kyanite rétrograde dans l'échantillon ne permettent pas de préciser si ces réactions rétrogrades se sont déroulées dans le champ de la kyanite ou de la sillimanite. Ces conclusions sont compatibles avec celles de Jordan et al. (2006) et Indares et al. (2008) qui ont documenté au nord du secteur B, un chemin P-T serré, croisant les réactions R1 et R2, puis Re-R2 et Re-R1 dans le champ de la kyanite.

\section{Métamorphisme rétrograde coïncidant avec les déformations D2 et D3}

\section{Secteurs C, D et E : paragneiss à sillimanite et phases de déformation D2 et D3}

Dans les secteurs C, D et E, les paragneiss contenant localement de la sillimanite, (affleurements 13SJ100 et 13SJ102) révèlent que la déformation D2 était synchrone à la cristallisation de sillimanite rétrograde (Fig. 1B et 7). La sillimanite est présente dans la matrice, mais également dans les plans de clivage de la biotite (Fig. 7A3 et 7B3). Un porphyroblaste de grenat xénomorphe et partiellement remplacé par la biotite, de type $\delta$ avec ombre de pression à sillimanite, indique un cisaillement senestre-inverse C2 (Fig. 7A1). La sillimanite est donc issue de la réaction Re-R2, et la déformation D2 s'est effectuée lors du métamorphisme rétrograde, dans le champ de la sillimanite. Ce minéral contenu dans la biotite défini des fabriques $\mathrm{C} / \mathrm{S}$ et $\mathrm{C}^{\prime}$ qui indiquent un cisaillement C2 senestre-inverse (Fig. 7A3), et confirme que la déformation D2 s'est effectuée dans le champ de la sillimanite. Ces conclusions sont compatibles avec celles de Jordan et al. (2006) qui ont documenté dans le secteur C, un chemin P-T rétrograde croisant la réaction Re-R2 dans le champ de la sillimanite, le chemin prograde n'ayant pu être défini. Ces évolutions métamorphiques, documentées dans les paragneiss à kyanite et à sillimanite et fondées sur des évidences des réactions R1, R2 et Re-R2, supportent la fusion partielle in-situ des 
paragneiss comme origine des leucosomes, et non l'injection de granite issus d'une fusion partielle ex-situ.

La déformation des grains de quartz permet de lier leur recristallisation aux déformations D1, D2 et D3. En effet, dans le secteur B, les grains de quartz dans la matrice et les leucosomes, de forme polygonale à lobée, ne sont pas allongés parallèlement à la S1 et n'ont pas d'extinction roulante (Fig. 6C à 6E). Dans les secteurs $\mathrm{C}$ et $\mathrm{D}$, le quartz contenu dans la matrice a une extinction roulante et est ponctuellement allongé parallèlement à la foliation S2 (Fig. 7A2 et 7A3), tandis que dans le secteur E affecté par la déformation D3, l'allongement du quartz parallèle à la S3 est plus commun dans la matrice et dans les leucosomes, quelques grains de quartz ont une extinction roulante. La recristallisation des grains de quartz est par conséquent postérieure à D1, en partie postérieure à D2 et synchrone à D3.

\section{Hornblendes rétrogrades syn-D2 dans un gabbro}

La relation entre le métamorphisme rétrograde et la phase de déformation inverse D2 est également indiquée par un dyke de gabbro, localisé au cœur de la Zone de cisaillement Relay, l'échantillon 12SJ27F (Fig. 1B et 8). Ce dyke recoupe la foliation S2 observée dans les gneiss archéens du Complexe de Ulamen, mais possède une linéation L2 plongeante vers le sud, parallèle à celle observée dans les gneiss du Complexe de Ulamen, marquée par la hornblende. Le clinopyroxène est corrodé indiquant qu'il ne faisait pas partie de la paragenèse d'équilibre, et il est localement présent en inclusion dans des grains de hornblende (Fig. 8A). Ces derniers, automorphes à subautomorphes (Fig. 8C), sont localement remplacés par la biotite et la chlorite (Fig. 8B). L'interprétation la plus probable de ces observations est une origine magmatique du clinopyroxène, ainsi qu'une mise en place du dyke de gabbro et une cristallisation de la hornblende pendant le métamorphisme rétrograde syn- à tardi-déformation D2. La datation de ce 
dyke de gabbro, décrite dans la section suivante géochronologie U/Pb (échantillon 12SJ27E), permettra donc de documenter l'âge de la mise en place de ce gabbro et l'âge de la déformation D2.

\section{Géochronologie U/Pb}

\section{Méthodes}

Les échantillons $12 \mathrm{SJ} 14$ et 12SJ27E ont été analysés à l'université Boise State en suivant la méthode LA-ICP-MS. Les grains de zircon ont été extraits en utilisant les techniques standards. Les grains des échantillons montrant des populations multiples ont été montés dans l'époxy et polis à leur cœur pour obtenir des images en cathodoluminescence (CL) à l'aide d'un microscope électronique à balayage JEOL JSM-1300. Le système LA-ICP-MS est composé d'un laser à 213 nm (New Wave) et d'un spectromètre ThermoElectron quadrupole ICP-MS (X-Series II). L'ablation laser a été effectuée avec un faisceau laser d'un diamètre de $30 \mu \mathrm{m}$ et d'une fréquence de $10 \mathrm{~Hz}$. L'échantillon vaporisé par le laser a été transporté jusqu'à la torche plasma dans un flux gazeux de He. Après un intervalle de $15 \mathrm{~s}$ pour mesurer le bruit de fond, le laser a été allumé et les données ont été acquises pendant 30 s pour les masses $\mathrm{Si}$ et $\mathrm{Zr}(5 \mathrm{~ms}),{ }^{49} \mathrm{Ti}$ et ${ }^{207} \mathrm{~Pb}(200 \mathrm{~ms})$, ${ }^{238} \mathrm{U},{ }^{232} \mathrm{Th},{ }^{202} \mathrm{Hg},{ }^{204} \mathrm{~Pb},{ }^{206} \mathrm{~Pb}$ et ${ }^{208} \mathrm{~Pb}(40 \mathrm{~ms})$ et pour les autres éléments à forte liaison atomique (high field strength elements) et les éléments de terres rares (ÉTR; $10 \mathrm{~ms}$ ). Le trou d'ablation était d'une profondeur de $25 \mu \mathrm{m}$, indépendamment du diamètre de celui-ci. La mise au point et le calcul des données ont été effectués en utilisant le logiciel interne à l'Université de Boise. Les âges U/Pb LA-ICP-MS utilisés dans ce travail, sont les moyennes pondérées des rapports isotopiques ${ }^{207} \mathrm{~Pb} /{ }^{206} \mathrm{~Pb}$ calculés à partir du logiciel Isoplot 4 (Ludwig, 2008). Ce rapport isotopique a été privilégié, car les incertitudes sont plus faibles pour des âges protérozoïques et la perte de plomb, fréquente pour des grains de zircon précambriens, affecte grandement le rapport 
${ }^{206} \mathrm{~Pb} /{ }^{238} \mathrm{U}$ (Mezger et al., 1997; Parrish et Noble, 2003). Un premier âge moyen pondéré a été calculé en utilisant les erreurs individuelles de chaque âge qui incluent les incertitudes de la constante de désintégration, mais n'incluent pas l'incertitude sur la calibration des standards. Ensuite, cette incertitude a été propagée dans l'erreur de l'âge pondéré moyen. Les incertitudes sur la calibration des standards sont estimées à $2,2 \%$ pour le rapport isotopique ${ }^{206} \mathrm{~Pb} /{ }^{238} \mathrm{U}$ et $0,7 \%$ pour le rapport isotopique ${ }^{207} \mathrm{~Pb} /{ }^{206} \mathrm{~Pb}$.

L'échantillon 12SJ14 a été ré-analysé par CA-ID-TIMS au GEOTOP à l'Université du Québec à Montréal. Les grains de zircon ont été extraits en utilisant les techniques standards. Ils ont ensuite été montés dans l'époxy et polis à leur cœur pour obtenir des images en CL à l'aide d'un microscope électronique à balayage. Des grains d'apparence comparable ont été traités par l'abrasion chimique et dissous par les techniques standards documentées dans Augland et al. (2015). Les solutions ont été déposées sur un filament et montées dans un spectromètre de masse VG354. Les analyses ont été effectuées au moyen d'un compteur d'ions Daly ou en mode "statique" avec des détecteurs Faraday multiples dont les paramètres de laboratoire sont décrits dans Augland et al. (2015). Les erreurs analytiques et les corrections ont été incorporées et propagées en utilisant une macro excel basé sur les algorithmes de Schmitz et Schoene (2007), et les âges sont calculés en utilisant Isoplot 4 (Ludwig, 2008).

Pour les deux méthodes d'analyse, les incertitudes citées sont d'ordre $2 \sigma$, et inclues l'incertitude sur la calibration des standards et les incertitudes de la constante de désintégration. Le groupement statistique est d'autant plus fiable que le mean square of the weighted deviates (MSWD) s'approche de 1,0 ou est plus petit que 1,0 et que la probability of fit (Prob. Fit.) s'approche de 1,0 (Ludwig, 2008). 


\section{Datation de la déformation inverse D2 dans le secteur B : Échantillon 12SJ14}

L'échantillon 12SJ14 est un leucosome prélevé sur un affleurement de paragneiss localisé au cœur d'une charnière de pli $\mathrm{P} 2$ au NO de la région à l'étude (Fig. 1B et 3). Cet échantillon à feldspath, quartz et biotite non déformés, ne contient ni aluminosilicate, ni grenat, bien que ce dernier minéral soit présent dans d'autres leucosomes de l'affleurement. Ce leucosome définit un rubanement migmatitique $\mathrm{D}$ 1, est plissé par la déformation D2, mais est interconnecté avec du leucosome se trouvant dans le plan de schistosité de plan axial D2 (Fig. 5A et 9A). Ces caractéristiques démontrent que le leucosome était liquide lors des deux phases de déformation et donc que sa cristallisation est synchrone à la schistosité de plan axial D2.

\section{Morphologie des grains de zircon}

Les grains de zircon ont une forme prismatique allongée et sont légèrement arrondis. Ils sont souvent fracturés, zonés et peuvent contenir des noyaux hérités (Fig. 9B). Ces noyaux, très variés, ont généralement de nombreuses zonations concentriques régulièrement tronquées, à brillance très élevée à très faible en $\mathrm{CL}$, et présentent rarement des zonations convolutes. Ces grains ont deux types de surcroissance; la première (groupe 1) entoure les noyaux hérités et est elle-même entourée de la deuxième (groupe 2) qui est présente sur certains grains de zircon. Le groupe 2 montre une brillance plus élevée en CL (Fig. 9B) et est plus mince que le groupe 1 (50 à $100 \mu \mathrm{m}$ versus inférieur à $200 \mu \mathrm{m})$. Le groupe 2 a des zonations peu marquées, souvent sectorielles et quelques fois concentriques, alors que le groupe 1 a plus fréquemment des zonations concentriques bien visibles (p. ex. zircon 47 sur la figure 9B). De rares grains homogènes ne présentent ni cœur hérité ni surcroissance et ont alors une brillance en CL et des zonations équivalentes à celles du groupe 1 ou du groupe 2 (Fig. 9B). Enfin, la forme allongée et 
les terminaisons pyramidales de l'ensemble des grains suggèrent une croissance dans un liquide (Corfu et al., 2003; Rubatto et Hermann, 2007).

\section{Analyse en terres rares}

33 analyses dont 15 dans le groupe 2, 16 dans le groupe 1 et 2 dans les noyaux hérités ont été effectuées. Huit de ces analyses sont différentes des autres (Fig. 9D4) et sont considérées comme des données aberrantes puisqu'elles ne sont pas reliées aux zones observées en CL et qu'elles ne concordent pas avec les groupes d'âge déterminés ci-dessous, ce qui peut être causé par des inclusions situées sous la surface des grains de zircon. La géochimie en éléments traces est similaire pour les deux groupes, mais le graphe Th versus $U$ permet de les distinguer et de confirmer les observations fondées sur l'imagerie par CL, puisqu'il démontre un enrichissement faible en Th et élevé en $U$ du groupe 1 et un appauvrissement de ces éléments pour le groupe 2 (Fig. 9C). La monazite, présente dans l'échantillon, séquestrant préférentiellement ces éléments U et Th (Bea et al., 1994), le groupe 2 a crû en présence de monazite au contraire du groupe 1. Les profils en ÉTR permettent de distinguer les surcroissances des noyaux hérités, puisque ces derniers ne sont pas appauvris en ÉTR lourdes et ont parfois une anomalie négative en Eu (tableau 2, Fig. 9D). Ces profils révèlent l'origine magmatique des noyaux hérités (Rubatto et Hermann, 2007). 23 analyses provenant des groupes 1 et 2 ont une géochimie en ÉTR semblable qui présente deux particularités (Fig. 9D2 à 9D4). La première est un appauvrissement en ÉTRlourdes qui indique que la croissance de zircon s'est effectuée pendant la formation de grenat. En effet, à haute pression et haute température, la distribution des ÉTR-lourdes s'effectue préférentiellement vers le grenat, le zircon aura alors un profil appauvri en ces éléments (Rubatto, 2002). La deuxième concerne l'absence d'anomalie négative en Eu qui démontre que la croissance du zircon est antérieure à la cristallisation de feldspath dans le liquide (Rubatto et Hermann, 
2007; Rubatto et al., 2006). Les températures calculées par le thermomètre Ti dans le zircon, donne des résultats similaires pour les deux groupes, soit environ $715^{\circ} \mathrm{C}$ (tableau 3 ), et ne permet donc pas de les différencier. Ainsi, les grains de zircon des groupes 1 et 2 sont typiques de zircon de haute pression qui ont cru lors de la formation de grenat avant la cristallisation de feldspath dans le liquide, car ils n'ont pas d'anomalie négative en Eu et ils ont un appauvrissement en ÉTRlourdes (Rubatto, 2002; Rubatto et Hermann, 2007). Toutefois, le groupe 2 a crû en présence de monazite à l'inverse du groupe 1, car il est appauvri en U et Th (Bea et al., 1994).

\section{Âges U/Pb sur zircon par LA-ICP-MS et CA-ID-TIMS}

Des dates $\mathrm{U} / \mathrm{Pb}$ ont été obtenues par LA-ICP-MS sur deux analyses de noyaux hérités, 11 analyses du groupe 1 et 12 analyses du groupe 2 . Les dates mesurées dans les noyaux hérités sont $2489 \pm 33$ et $2401 \pm 59$ Ma, alors que les âges pondérés moyens calculés pour le groupe 1 (Fig. 9D2) et le groupe 2 (Fig. 9D3) sont respectivement de $1008 \pm 18 \mathrm{Ma}(\mathrm{MSWD}=1,8$; Prob. Fit $=$ $0,06)$ et de $995 \pm 21$ Ma (MSWD = 0,89; Prob. Fit $=0,55)$. Les analyses du groupe 2 sont concordantes, tandis que celles du groupe 1 sont discordantes de 1 à $7 \%$.

Six autres analyses obtenues par CA-ID-TIMS sont concordantes ou discordantes jusqu'à 1,7\% et se divisent en deux ensembles d'âge différent (tableau 5, Fig. 9F). Le plus vieux groupe, obtenu sur des pointes cassées, donne un âge concordia de $1002 \pm 2$ Ma (MSWD =0,117), calculé d'après deux analyses concordantes ( ${ }^{\circ} 1$ et 5 ). Le plus jeune groupe, obtenu sur des fragments d'autres grains cassés, donne un âge concordia de $986 \pm 4$ Ma (MSWD =0,027), calculé sur une analyse concordante $\left(n^{\circ} 6\right)$ et supporté par l'âge $985 \pm 7$ Ma $(\mathrm{MSWD}=0,049)$ de l'intercepte supérieur des analyses $n^{\circ} 3$ et 6 qui chevauche l'âge concordia de l'analyse $n^{\circ} 6$. Les analyses $n^{\circ} 2$ et 4 sont discordantes avec des âges ${ }^{207} \mathrm{~Pb} /{ }^{206} \mathrm{~Pb}$ plus vieux que ceux provenant des autres analyses, indiquant probablement une portion de noyaux hérités dans le matériel, elles ont donc 
été ignorées pour le calcul des âges. Étant donné que les grains en CL n'ont que deux surcroissances d'âge grenvillien, l'ensemble le plus vieux correspond vraisemblablement au groupe 1 et l'ensemble le plus jeune au groupe 2. Ainsi, les résultats CA-ID-TIMS confirment et augmentent la précision des résultats LA-ICP-MS.

\section{Interprétation}

La forme prismatique des grains de zircon de l'échantillon 12SJ14 indique une croissance dans un liquide de fusion partielle, alors que leur profil appauvri en ÉTR-lourdes indique une croissance synchrone à celle du grenat présent dans le paragneiss et les leucosomes environnants. Puisque la forme du zircon supporte l'interprétation générale de croissance en présence de liquide (Vavra et al., 1999; Rubatto et al., 2001) l'âge du groupe 1 (1002 \pm 2 Ma) est interprété comme l'âge initial du métamorphisme de haute température et haute pression et de la fusion partielle par R2. Cet évènement est vraisemblablement synchrone à la déformation D1, puisque sa foliation est définie par un rubanement migmatitique (Fig. 5A2) et que la cristallisation de kyanite et biotite progrades est anté-D1 (Fig. 6). L'âge du groupe 2 (986 $\pm 4 \mathrm{Ma}$ ) est interprété comme l'âge de cristallisation du leucosome localisé dans la charnière d'un pli P2. Le profil en ÉTR de ce groupe indique une cristallisation synchrone à celle du grenat et antérieure à la cristallisation de feldspath dans le liquide, par R2, près du paroxysme du métamorphisme. Ainsi, bien qu'il soit communément admis que les grains de zircon croient spécifiquement pendant la cristallisation de liquide dans les systèmes anatectiques (p. ex. Harley et al., 2007; Kelsey et al., 2008; Yakymchuk et Brown, 2014), des études récentes ont démontrés une croissance des zircon lors du chemin prograde et au paroxysme du métamorphisme (p. ex. Gervais et Crowley, 2017). Enfin, si les grains de zircon avaient cru lors de la cristallisation des leucosomes par la réaction Re-R2, le remplacement du grenat aurait libéré les ÉTR-lourdes dans le liquide et la 
cristallisation de plagioclase aurait appauvri le liquide en Eu. Le profil en ÉTR des grains de zircon aurait alors été enrichi en ÉTR-lourdes avec une anomalie négative en Eu, et serait donc contraire aux profils documentés des groupes 1 et 2 . La concentration en $U$ et $T h$ du groupe 2 indique également une cristallisation synchrone à celle de la monazite supportée par les âges $\mathrm{U} / \mathrm{Pb}$ de 995-985 Ma sur monazite (Jordan et al., 2006) similaires à celui des grains de zircon de ce groupe 2. Cet âge de $986 \pm 4$ Ma correspond donc vraisemblablement au début de la déformation D2, ainsi qu'au tout début du chemin rétrograde, bien avant la réaction Re-R2 documentée dans d'autres secteurs C et D, par la sillimanite associée aux structures D2 (Fig. 7).

L'absence de surcroissance anté-Rigolet sur les grains de zircon ayant des noyaux hérités montre, en outre qu'il n'y a pas eu de croissance métamorphique de zircon entre le dépôt du Groupe de Gagnon à 1875 Ma (Moukhsil et al., 2013) et l'orogénie grenvillienne. En effet, bien que les grains de zircon datés ne proviennent pas d'un paléosome mais d'un leucosome contenant en comparaison peu de noyaux hérités, les images en CL de $\sim 200$ grains ne montrent pas de surcroissance, différente des groupes 1 et 2 , tronquant les $\sim 40$ noyaux hérités imagés. En outre, la forme majoritairement arrondie de ces noyaux hérités privilégie une origine détritique, et donc un âge pour ces noyaux antérieur au dépôt des roches méta-sédimentaires du Groupe de Gagnon. Enfin les datations effectuées par le MERNQ sur des paléosomes, soit un quartzite et un gneiss tonalitique, supportent cette hypothèse puisque les âges discordants se répartissent sur une ligne de mélange par échantillon, avec un intercepte supérieur archéen ou à $1875 \mathrm{Ma}$ et un intercepte inférieur grenvillien (Davis et Dion, 2012a et b). Ainsi, aucun épisode métamorphique n'a été enregistré dans les grains de zircon, entre le dépôt du Groupe de Gagnon et l'orogène grenvillien. 


\section{Datation de la déformation inverse D2 dans le secteur D : Échantillon 12SJ27E}

L'échantillon 12SJ27E est une pegmatite localisée à proximité de la zone de cisaillement Relay (Fig. 1B). Sur l'affleurement 12SJ27, un dyke de gabbro composite recoupe la foliation S2, mais expose une linéation identique en attitude et en minéralogie à celle L2 (Fig. 10A1 et 10A3). Ce dyke s'est également mis en place dans la foliation S2, où il contient une pegmatite dont la cristallisation est synchrone à celle du gabbro (Fig. 10A2 et 10B). En effet, il est possible de retrouver des fragments arrondis, soit de gabbro dans la pegmatite, soit de pegmatite dans le gabbro (Fig. 10A2), ce qui révèle un mélange de magma (Vernon et al., 1988). Les textures métamorphiques et les structures de ce gabbro, décrites précédemment, sont une linéation L2 définie par de la hornblende rétrograde à inclusions de clinopyroxène instable (lame mince 12SJ27F, Fig. 8). Ce gabbro et cette pegmatite sont donc synchrones, mais tardi-D2, et cette déformation est associée au métamorphisme rétrograde.

\section{Morphologie des grains de zircon}

Les grains de zircon ont une forme prismatique, des bordures bien préservées et sont souvent zonés. Ils peuvent contenir des noyaux hérités et deux types de surcroissance (Fig. 10C). La première (groupe 1) entoure les noyaux hérités et est elle-même entourée de la deuxième (groupe 2) qui est présente sur certains grains. Le groupe 2 montre une brillance en CL plus élevée, et est plus mince que le groupe $1(100 \mu \mathrm{m}$ versus inférieur à $300 \mu \mathrm{m})$. Les zonations, très bien marquées, sont souvent sectorielles et quelques fois concentriques. Certains grains de zircon homogènes $(\sim 10 \%)$, inclus dans le groupe 1 , ne présentent ni noyaux hérités ni surcroissance et ont une brillance en CL équivalente à celle de ce groupe (Fig. 10C). Les faces cristallines bien définies des grains et leurs zonations très nettes indiquent qu'ils sont magmatiques. 


\section{Analyses en terres rares}

Pour cet échantillon 38 analyses, dont deux écartées, ont été effectuées dans le groupe 2 (12 analyses) et le groupe 1 (24 analyses). Cinq de ces analyses sont différentes des autres (Fig. 10D3) et sont considérées comme des données aberrantes puisqu'elles ne sont pas reliées aux zones observées en CL et qu'elles ne concordent pas avec les groupes d'âge déterminés cidessous, ce qui peut être causé par des inclusions situées sous la surface des grains de zircon. Les 33 analyses identiques, ont des profils en ÉTR caractérisés par une anomalie positive en Ce, une anomalie négative en Eu et l'absence d'anomalie négative en ÉTR lourdes (tableau 2, Fig. 10D). Ces grains de zircon ont donc crû pendant la cristallisation de plagioclase et présentent des profils typiques de zircon magmatique (Rubatto et Hermann, 2007).

\section{Âges U/Pb sur zircon par LA-ICP-MS}

Des dates $\mathrm{U} / \mathrm{Pb}$ ont été obtenues sur 19 analyses du groupe 1 et 9 analyses du groupe 2, et seuls les résultats par LA-ICP-MS sont présentés, car aucun résultat probant n'a été obtenu par CA-IDTIMS. Les âges pondérés moyens calculés pour le groupe 1 et le groupe 2 sont respectivement de $1001 \pm 16 \mathrm{Ma}(\mathrm{n}=19 ; \mathrm{MSWD}=1,4$; Prob. Fit =0,13) et de $961 \pm 22 \mathrm{Ma}(\mathrm{n}=9 ;$ MSWD = 1,17; Prob. Fit $=0,31)$. Toutes les analyses du groupe 2 sont concordantes, mais celles du groupe 1 sont discordantes de 1 à $12 \%$. Bien que les profils en ÉTR des groupes 1 et 2 soient similaires et qu'il ne soit pas possible de départager ces âges sur une base statistique, les images CL et leurs âges attestent qu'il s'agit de deux groupes différents. L'âge de $1001 \pm 16 \mathrm{Ma}$ est synchrone à l'anatexie de la Ceinture parautochtone dans la région d'étude, puisque cette fusion partielle par la réaction R2 a été datée à 1002 Ma dans les roches méta-sédimentaires de l'échantillon 12SJ14. Cet âge de 1001 Ma pourrait donc être hérité et correspondrait à la croissance de zircon dans les migmatites, une partie de ce magma granitique et de ses grains de zircon contenus étant ensuite 
injectée sous forme de dyke de pegmatites. L'âge le plus jeune de $961 \pm 22$ Ma est interprété comme l'âge de cristallisation de la pegmatite. Ainsi, cette datation démontre que certains gabbros ne sont pas archéens mais ont un âge grenvillien tardi-Rigolet.

\section{Discussion}

\section{Sommaire des résultats}

La figure 11 résume l'analyse des données structurales, métamorphiques et géochronologiques de la Ceinture parautochtone. Trois, possiblement quatre phases de déformation y sont présentes :

1) La déformation D1, présente uniquement au NO de la région d'étude, est caractérisée par une foliation NO-SE portant une linéation plongeante vers le SE (Fig. 2). Cette phase de déformation n'est pas archéenne, car elle affecte les méta-sédiments du Groupe de Gagnon qui sont protérozoïques. En outre, la définition de la foliation S1 par un rubanement migmatitique (Fig. 5A), et la présence de kyanite subparallèle à la foliation S1 dans des leucosomes (Fig. 6A) indiquent que la déformation D1 s'est effectuée en présence de liquide issu de la réaction $\mathrm{R} 1$ dans le champ de la kyanite. Cette déformation aurait continué lors de la réaction $\mathrm{R} 2$ pour former du grenat, toujours dans le champ de la kyanite. De plus, dans l'échantillon 12SJ14, les profils en ÉTR des grains de zircon du groupe 1 possèdent une absence d'anomalie négative en Eu et un appauvrissement en ÉTR-lourdes qui sont caractéristiques d'une croissance synchrone à celle du grenat et antérieure à celle du plagioclase dans le liquide, à haut grade métamorphique et plus particulièrement à haute pression (Rubatto, 2002; Rubatto et Hermann, 2007). Ce grade métamorphique est confirmé par l'échantillon 13SJ182, qui indique un chemin P-T de haute pression, ayant croisé les réactions R1, R2 puis Re-R2 et Re-R1 dans le champ de la kyanite. Ce chemin P-T est identique à celui serré, décrit par Jordan et al. (2006) et 
atteignant des pressions de 1250-1300 $\mathrm{MPa}$ et des températures de $815-830^{\circ} \mathrm{C}$. Puisqu'il est généralement admis que les surcroissances prismatiques de zircon dans les paragneiss métamorphisés au faciès granulite sont liées à l'anatexie (Vavra et al., 1999; Rubatto et al., 2001; Gervais et Crowley, 2017), et que le profil en ÉTR et la forme des grains de zircon du groupe 1 concordent avec cette hypothèse, l'âge du groupe 1 de $1002 \pm 2 \mathrm{Ma}$ (Fig. 9 et 11) coïncide probablement avec la phase de déformation D1 près du paroxysme du métamorphisme.

2) Nous avons cartographié un gradient de la déformation D2 qui passe de peu à fortement pénétrative du NO de la région d'étude, vers la zone de cisaillement Relay, une structure D2, et vers l'ABT. Cette déformation est caractérisée par une linéation plongeant vers le SSO qui demeure constante malgré le plissement de la foliation (Fig. 2). Le sens de cisaillement est majoritairement senestre-inverse (Fig. 5D). Le leucosome 12SJ14 est précoce à syn-déformation D2 (Fig. 9) alors que la pegmatite 12SJ27E est synchrone à tardi-déformation D2 (Fig. 10). D'après la datation de ces deux échantillons, cette phase de déformation D2 a débuté vers $986 \pm 4$ Ma, simultanément à la cristallisation de zircon de groupe $2 \mathrm{du}$ leucosome 12SJ14. Elle s'est ensuite poursuivie jusqu'à $961 \pm 22$ Ma, coïncidant avec la cristallisation de la pegmatite 12SJ27E. Sans datation plus précise, il est difficile d'affirmer que la déformation s'est prolongée aussi tardivement, mais cela est probable, puisque la comparaison des analyses par LA-ICP-MS et CA-ID-TIMS sur l'échantillon 12SJ14 (Fig. 9) a démontré l'exactitude des résultats obtenus par LA-ICPMS. Étant donné que les deux échantillons ont été datés ensemble par LA-ICP-MS, l'erreur de calibration peut être supprimée, l'erreur relative entre les deux échantillons devient significative, et la différence relative entre les deux âges est importante. Une fois 
cette erreur de calibration supprimée, le leucosome 12SJ14 donne donc un âge de $1008 \pm 9$ Ma pour la déformation D1 et un âge de $995 \pm 11$ Ma pour le début de la déformation D2, alors que la pegmatite donne un âge tardi-D2 de $961 \pm 11$ Ma (Fig. 11). Dans le secteur B, la déformation D1 a donc commencé au plus tard à 999 Ma alors que la déformation D2 a commencé au plus tard à $984 \mathrm{Ma}$, et dans le secteur $\mathrm{D}$, la déformation D2 a fini au plus tôt à 972 Ma. Par conséquent, en supposant que dans ces deux secteurs, ces déformations se sont chacune déroulées au même moment, le cisaillement inverse, marqué d'abord par un mouvement D1 vers le NO puis par un mouvement D2 vers le NNE, aurait duré $27 \mathrm{Ma}$ minimum dont 12 Ma pour la déformation D2 uniquement. Ces déformations pourraient cependant être progressives, tel que documentées dans le prisme orogénique de la Ceinture parautochtone dans la Province de Grenville oriental (Labrador ouest; van Gool et al., 2008), avec par exemple une déformation D1 dans le secteur D synchrone à une déformation D2 dans le secteur B, la durée de la déformation D2 serait alors moindre, mais celle de la déformation D1 de 17 Ma resterait valide pour les deux hypothèses. Les éléments de cette étude ne sont pas suffisants pour répondre à ce problème de déformations progressives ou non progressives, car dans cette région, notre étude ne permet de définir les processus d'enfouissement ou d'exhumation de la Ceinture parautochtone, à l'origine des déformations, l'âge de l'ABT n'ayant pas été documenté.

Cette étude permet également d'associer la déformation D2 à un chemin P-T. Premièrement, dans l'échantillon 13SJ100B, la transformation d'un porphyroblaste de grenat en biotite et sillimanite indique que le cisaillement C2 s'est effectué lors du chemin rétrograde, postérieurement à la réaction Re-R2 dans le champ de la sillimanite (Fig. 7). Bien qu'il soit impossible de préciser si l'anatexie s'est effectuée dans le champ de la 
kyanite, comme pour l'ouest de la région d'étude, ou dans le champ de la sillimanite, ce qui indiquerait la présence de deux niveaux structuraux, nos données confirment le chemin rétrograde reconstruit par Jordan et al. (2006) dans l'est de la région à l'étude.

Deuxièmement, dans l'échantillon 12SJ14, les grains de zircon d'âge $986 \pm 4$ Ma montrent un profil en ÉTR ayant une absence d'anomalie négative en Eu et un appauvrissement en ÉTR-lourdes, similaire au profil du groupe 1. Néanmoins, les grains de zircon sont appauvris en $\mathrm{U}$ et $\mathrm{Th}$, au contraire du groupe 1 plus vieux. Ces profils indiquent une évidence de croissance des grains de zircon, dans le liquide, synchrone à celle du grenat et de la monazite (Fig. 9; Bea et al., 1994) et antérieure à celle de plagioclase. Ce groupe 2 a donc fort probablement cru sur le chemin rétrograde, à proximité du paroxysme métamorphique et simultanément à la cristallisation de monazite dans le leucosome, cristallisation confortée par la présence de monazite dans l'échantillon daté, et par les âges $\mathrm{U} / \mathrm{Pb}$ similaires de 995-985 Ma pour les grains de monazite (Jordan et al., 2006) et le groupe 2. Cette évolution pétrogénétique est très semblable à celle documentée dans l'équivalent de la Ceinture parautochtone de la Cordillère canadienne (Gervais et Crowley, 2017).

Troisièmement, l'échantillon de gabbro 12SJ27F syn-déformation D2 dont la cristallisation est synchrone à celle de la pegmatite 12SJ27E, possède également de l'amphibole rétrograde parallèle à la linéation L2 (Fig. 10). Cette amphibole s'est donc formée à $961 \pm 22$ Ma. Enfin, les grains de quartz ne sont pas déformés dans les secteurs où seule la déformation D1 est présente et peu ou pas déformés dans les secteurs où la phase D2 prédomine, signifiant que du liquide était présent lors de ces deux déformations. 
Somme toute, la déformation D2 s'est produite lors d'un chemin P-T rétrograde postérieurement à la réaction Re-R2, mais toujours à l'état supra-solidus.

3) La déformation D3 a été observée seulement sur certains affleurements dans la partie sud de la région d'étude, vers l'ABT (Fig. 11). Cette phase de déformation a complètement transposé les structures D2 et réorienté la linéation qui devient faiblement plongeante vers l'OSO (Fig. 2). Un leucosome syn-D3 révèle que cette déformation est associée à un cisaillement dextre-normal et s'est produite en présence de fusion partielle (Fig. 5G). Par conséquent, toutes les phases de déformation se sont déroulées en présence de fusion partielle; de ce fait, le métamorphisme d'anatexie est également rigolet.

\section{Possibilité d'une déformation grenvillienne anté-D1}

Il est plausible de supposer qu'une déformation grenvillienne antérieure à la déformation D1 ait existée dans la Ceinture parautochtone, au SO du réservoir Manicouagan. En effet, des structures typiques de la direction de convergence de l'orogène du Grenville, correspondant à la première phase de déformation grenvillienne cartographiée, ont été documentées à proximité du Front de Grenville dans la Ceinture parautochtone de la Province de Grenville orientale, et sont caractérisées par une vergence NO et des fabriques planaires parallèles à l'orogène (Rivers, 1983a; Brown et al., 1992; van Gool et al., 2008). Étant donné que dans la région à l'étude, 1) ces structures typiques de la convergence grenvillienne n'ont pu être observées puisqu'elles sont différentes des structures D1, ces dernières ayant une vergence NO mais des fabriques planaires perpendiculaires à l'orogène grenvillien; 2) que la déformation D1 est synchrone au début d'anatexie liée la réaction $\mathrm{R} 1$, puis à la réaction $\mathrm{R} 2$, et 3) que D1 est synchrone à postérieure à la cristallisation de kyanite et biotite progrades parallèles à la $\mathrm{S} 1$ puisque ces minéraux présentent des extinctions roulantes; il est plausible de supposer qu'une déformation antérieure, associée au 
métamorphisme prograde précédent l'anatexie syn-D1 ait existée. D1 n'était donc éventuellement pas précédée d'un continuum, mais d'une phase de déformation grenvillienne distincte caractérisée par des structures planaires NE-SO à vergence NO typiques de la direction de convergence de l'orogène du Grenville. Cette déformation antérieure aurait pu amorcer l'enfouissement de la Ceinture parautochtone et notamment celui du Groupe de Gagnon situé vraisemblablement dans la croûte superficielle au début de l'orogénie grenvillienne, avant 1002 Ma. De nouvelles études sont cependant nécessaires pour tester cette hypothèse.

\section{Stratégie de datation géochronologique dans les terrains de haut grade métamorphique précambriens}

Cette contribution illustre l'importance d'interpréter les analyses géochronologiques avec la plus grande prudence dans les terrains de haut grade métamorphique précambriens tel que la Province de Grenville. Dans la Province de Grenville centrale, nos résultats supportent l'hypothèse, pour notre région d'étude, d'une déformation archéenne oblitérée lors du métamorphisme grenvillien au faciès granulite. Ces résultats suggèrent également que le paradoxe énoncé précédemment est causé par les méthodes de datations employées qui rendent l'interprétation des âges difficile. En effet, notre étude établit que la plus vieille déformation documentée, soit D1, coïncide avec l'anatexie des roches parautochtones au Rigolet. La foliation formée lors de D1 est pénétrative à l'ouest de la région d'étude et a été transposée par D2 à proximité de l'ABT (Fig. 1B, 3, 5 et 11A). Il n'existe donc aucune évidence d'une déformation archéenne ou paléoprotérozoïque de hautgrade métamorphique au SO du réservoir Manicouagan. Ce postulat requiert donc une réinterprétation de l'âge du dyke de granite qui recoupe la gneissosité de l'encaissant dans le secteur D dominé par D2 (Dunning et Indares, 2010). Ainsi, l'âge discordant d'intercepte supérieur de $\sim 1741$ Ma correspond probablement à un âge hérité et non à un âge de 
cristallisation, ce dernier se mesurant vraisemblablement dans des surcroissances. En outre, les deux grains de zircon montrés par ces auteurs sont constitués de cœurs entourés de telles surcroissances (grains 289-1 et 289-2 sur la figure 5 dans Dunning et Indares, 2010). Cet exemple démontre l'utilité d'utiliser des méthodes de datations combinant haute résolution spatiale et haute précision pour l'analyse géochronologique des terrains de haut grade métamorphique précambriens. De surcroit, il engendre inévitablement des questionnements relatifs à l'interprétation des nombreux âges discordants qui ont été utilisés pour contraindre la chronologie des déformations dans la Province de Grenville.

\section{Remobilisation du socle et de sa couverture méta-sédimentaire}

Les résultats de cette étude impliquent que dans les gneiss du socle appartenant au Complexe de Ulamen, la déformation archéenne a été complètement oblitérée lors du métamorphisme grenvillien au faciès granulite. Cette constatation est compatible avec celle obtenue notamment dans la Province de Grenville orientale, où une oblitération progressive des fabriques archéennes a été documentée vers le cœur de l'orogène et les faciès métamorphiques plus élevés (Rivers, 1983a, 1983b; Owen et al., 1986; 1988; Brown et al., 1991, 1992; Daigneault et Allard, 1994; Krogh et al., 2002; van Gool et al., 2008), mais détonne avec celle obtenue notamment dans la Province de Grenville occidentale, où une fabrique archéenne est apparemment préservée malgré un métamorphisme de haut grade (Berclaz et al., 1995; Bethune, 1997; Martignole et Martelat, 2005). Tel que discuté ci-dessus, il serait cependant intéressant d'effectuer des datations modernes à ces endroits afin de déterminer si cette préservation d'une fabrique archéenne est réelle.

Malgré cette oblitération grenvillienne, il existe des indices d'évènements tectoniques antérieurs dans la région d'étude. Par exemple, dans le secteur B (Fig. 3), la juxtaposition de contacts socle - 
méta-sédiments de nature contrastée suggère la présence de chevauchements précoces qui auraient été complètement transposés. D'autre part, Jordan et al. (2006) ont rapporté la présence de monazite paléoprotérozoïque $(\sim 1738 \mathrm{Ma})$ dans les paragneiss du Groupe de Gagnon. L'interprétation de ces âges par les auteurs est une origine métamorphique, mais ce métamorphisme cryptique devait être d'un grade relativement faible puisqu'il n'y a pas eu de croissance de zircon, liée à l'anatexie, dans les paragneiss, les quartzites et les gneiss archéens investigués dans cette étude et ailleurs dans la région d'étude (Davis et Dion, 2012a et b; Moukhsil et al., 2013). Si cette croissance de monazite pourrait avoir être générée par la réaction déshydratation-fusion de l'allanite dans les métapélites, au faciès amphibolite inférieur (Smith et Barreiro, 1990); il serait pertinent d'effectuer une étude moderne, dans les paragneiss, qui combinerait la composition géochimique et l'analyse isotopique de monazite à celles de grenat, afin de confirmer l'existence de cet évènement et s'il y a lieu, dans préciser la nature.

\section{Conclusion}

En conclusion, contrairement à ce qui a été observé dans certaines régions du Front de Grenville où des fabriques antérieures à l'orogène de Grenville ont été préservées, à proximité de l'ABT au SO du réservoir Manicouagan où le métamorphisme est de plus haut grade, toutes les déformations étudiées ont un âge comprit entre 1002 et 961 Ma et sont majoritairement inversedécrochantes. La durée de cet évènement compressif, tardi-orogénique, est supérieure à 27 millions d'années et a débuté avant 1002 Ma, car cet âge correspond à l'anatexie par la réaction de déshydratation-fusion de la biotite dans les paragneiss, et que cette durée ne comptabilise pas l'enfouissement de la Ceinture parautochtone jusqu'à cette anatexie. Ainsi, contrairement aux hypothèses précédemment admises, la phase Rigolet a été un évènement prolongé, tout au moins 
localement, et malgré sa durée moindre que celle de l'Ottawan estimée à 70 millions d'années minimum (Rivers, 2008).

Dans la région d'étude, le mouvement inverse de l'ABT n'a pas été enregistré par la croissance de zircon et de monazite, car les âges sur zircon et monazite connus, coïncident aux phases de déformations D1 et D2 et que le mouvement inverse de l'ABT ne correspond à aucune des trois déformations cartographiées. Ainsi, une étude similaire de la Ceinture allochtone serait nécessaire pour documenter l'âge de l'ABT. En outre, une telle étude, combinée à une analyse régionale des linéaments géophysiques, permettrait de déterminer si l'incurvation de l'ABT autour du réservoir Manicouagan est probablement issue d'un plissement par la phase D1 documentée dans cette étude, et ainsi vérifier l'oblitération du mouvement inverse de l'ABT par les déformations D1 à D3.

D'après les résultats de cette étude, le degré de superposition des structures et du métamorphisme grenvillien sur les indices d'évènements tectoniques précédents et le degré d'oblitération des déformations antérieures seraient des indicateurs de l'effet potentiellement important du remaniement grenvillien. Il est ainsi possible de supposer qu'à proximité du Front de Grenville, les déformations principales seraient archéennes, hudsoniennes ou makkovikiennes alors que les structures grenvilliennes seraient secondaires et associées à un métamorphisme grenvillien au faciès schiste vert-amphibolite. Au contraire, vers l'ABT, les structures principales sont tardigrenvilliennes et associées à un métamorphisme granulite, les déformations antérieures dont le mouvement inverse, et ottawan de l'ABT étant complétement oblitérées. Dans la Ceinture parautochtone, le remaniement grenvillien affecterait aussi bien les fabriques anté-grenvilliennes que celles ottawans et serait donc faible à proximité du Front de Grenville alors qu'il augmenterait vers le SE jusqu'à la transposition complète à l'ABT. 
Cette contribution illustre également la complexité de la datation dans les terrains de haut grade métamorphique précambrien. Notre stratégie de datation qui consiste à dater des échantillons syndéformations en combinant la géochimie en éléments traces et la haute résolution spatiale du LAICP-MS permettant de distinguer les populations de zircon, à la haute précision du CA-ID-TIMS, est justifiée. Elle permet effectivement de contraindre l'origine du zircon tout en discriminant deux phases de déformations dont la différence d'âge est statistiquement non significative. Nous suggérons de réinvestiguer plusieurs endroits de la Ceinture parautochtone en utilisant une stratégie similaire pour déterminer l'étendue de la remobilisation du socle archéen et de sa couverture méta-sédimentaire.

Enfin, d'une perspective plus générale, l'association des profils en ÉTR aux âges in-situ des grains de zircon de l'échantillon 12SJ14 implique une croissance à proximité du paroxysme du métamorphisme. Ces résultats sont contraires aux prédictions théoriques qui stipulent une dissolution du zircon dans les liquides anatectiques, lors de tout chemin prograde (p. ex. Kelsey et al., 2008; Yakymchuk et Brown, 2014), mais supportent les conclusions de Gervais et Crowley (2017) et de Vavra et al. (1999) effectuées sur des roches similaires. De nouvelles études sont requises pour élucider par quel processus le zircon peut se former lors de chemins progrades ou près du pic métamorphique.

\section{Remerciements}

Ce travail est une partie de la thèse de doctorat de SJ supervisée par FG. Le Ministère de l'Énergie et des Ressources Naturelles du Québec est remercié pour son soutien financier et technique lors des travaux de terrain et des analyses. A. Indares et T. Rivers sont remerciés pour leur examen détaillé et très constructif de ce manuscrit qui ont permis d'améliorer la qualité et la clarté des propos. 


\section{Références}

Augland, L.E., Moukhsil, A., Solgadi, F., et Indares, A. 2015. Pinwarian to Grenvillian magmatic evolution in the central Grenville Province: new constraints from ID-TIMS U-Pb ages and coupled Lu-Hf S-MC-ICP-MS data. Canadian Journal of Earth Sciences, 52: 701-721. doi:10.1139/cjes-2014-0232.

Bea, F., Pereira, M.D., et Stroh, A. 1994. Mineral/leucosome trace-element partitioning in a peraluminous migmatite (a laser ablation-ICP-MS study). Chemical Geology, 117: 291-312.

Berclaz, A., Hébert, R., et Rocheleau, M. 1995. La zone tectonique du front de Grenville à l'est de Louvicourt, Québec: exhumation de la croûte archéenne pendant l'orogénie grenvillienne. Canadian Journal of Earth Sciences, 32: 1899-1920.

Berthé, D., Choukroune, P., et Jegouzo, P. 1979. Orthogneiss, mylonite and non coaxial deformation of granites: the example of the South Armorican Shear Zone. Journal of Structural Geology, 1: 31-42. doi:10.1016/0191-8141(79)90019-1.

Bethune, K.M. 1997. The Sudbury dyke swarm and its bearing on the tectonic development of the Grenville Front, Ontario, Canada. Precambrian Research, 85: 117-146. doi:10.1016/S03019268(96)00052-6.

Brown, D., van Gool, J., Calon, T., et Rivers, T. 1991. The geometric and kinematic development of the Emma Lake thrust stack, Grenville Front, southwestern Labrador. Canadian Journal of Earth Sciences, 28: 136-144. doi:10.1139/e91-012.

Brown, D., Rivers, T., et Calon, T. 1992. A structural analysis of a metamorphic fold-thrust belt, northeast Gagnon terrane, Grenville Province. Canadian Journal of Earth Sciences, 29: 19151927. doi:10.1139/e92-149.

Carr, S.D., Easton, R.M., Jamieson, R.A., et Culshaw, N.G. 2000. Geologic transect across the Grenville orogen of Ontario and New York. Canadian Journal of Earth Sciences, 37: 193-216. doi:10.1139/e99-074.

Clarke, P.J. 1977. Région de Gagnon. Ministère des Richesses Naturelles, Québec, RG 178. 
Connelly, J.N., et Heaman, L.M. 1993. U/Pb geochronological constraints on the tectonic evolution of the Grenville Province, western Labrador. Precambrian Research, 63: 123-142.

Connelly, J.N., Rivers, T., et James, D.T. 1995. Thermotectonic evolution of the Grenville Province of western Labrador. Tectonics, 14: 202-217.

Corfu, F., Hanchar, J.M., Hoskin, P.W.O., et Kinny, P. 2003. Atlas of zircon textures. Reviews in Mineralogy and Geochemistry, 53: 469-500. doi:10.2113/0530469.

Corrigan, D., Rivers, T., et Dunning, G. 2000. U-Pb constraints for the plutonic and tectonometamorphic evolution of Lake Melville terrane, Labrador and implications for basement reworking in the northeastern Grenville Province. Precambrian Research, 99: 65-90. doi:10.1016/S0301-9268(99)00054-6.

Crowley, J.L. 1999. U-Pb geochronologic constraints on Paleoproterozoic tectonism in the Monashee complex, Canadian Cordillera: Elucidating an overprinted geologic history. Geological Society of America Bulletin, 111: 560-577.

Crowley, J.L., Ghent, E.D., Carr, S.D., Simony, P.S., et Hamilton, M.A. 2003. Multiple thermotectonic events in a continuous metamorphic sequence, Mica Creek area, southeastern Canadian Cordillera. American Mineralogist, 88: 930-930.

Crowley, J.L., Brown, R.L., Gervais, F., et Gibson, H.D. 2008. Assessing inheritance of zircon and monazite in granitic rocks from the Monashee Complex, Canadian Cordillera. Journal of Petrology, 49: 1915-1929. doi:10.1093/petrology/egn047.

Daigneault, R., et Allard, G.O. 1994. Transformation of Archean structural inheritance at the Grenvillian Foreland Parautochthon Transition Zone, Chibougamau, Quebec. Canadian Journal of Earth Sciences, 31: 470-488. doi:10.1139/e94-043.

Davis, D.W., et Dion, C. 2012a. Datations LA-ICPMS d'échantillons recueillis en 2011-2012 par Géologie Québec. Ministère des Ressources Naturelles et de la Faune, Québec, MB 2012-09.

Davis, D.W., et Dion, C. 2012b. Datations ID-TIMS d'échantillons recueillis en 2011-2012 par Géologie Québec. Ministère des Ressources Naturelles et de la Faune, Québec, MB 2012-07. 
Dewey, J.F., Holdsworth, R.E., et Strachan, R.A. 1998. Transpression and transtension zones. Geological Society, London, Special Publications, 135: 1-14.

Dunning, G., et Indares, A. 2010. New insights on the 1.7-1.0 Ga crustal evolution of the central Grenville Province from the Manicouagan - Baie Comeau transect. Precambrian Research, 180: 204-226. doi:10.1016/j.precamres.2010.04.005.

D’Amours, I., et Intissar, R. 2012a. Levé magnétique aéroporté dans le secteur Manic-Outardes, Province de Grenville. Ministère des Ressources Naturelles et de la Faune, Québec, DP 2012-03.

D’Amours, I., et Intissar, R. 2012b. Levé magnétique aéroporté dans le secteur du lac SainteAnne, Province de Grenville. Ministère des Ressources Naturelles et de la Faune, Québec, DP 2012-02.

Foster, G., Parrish, R.R., Horstwood, M.S., Chenery, S., Pyle, J., et Gibson, H.D. 2004. The generation of prograde $\mathrm{P}-\mathrm{T}-\mathrm{t}$ points and paths; a textural, compositional, and chronological study of metamorphic monazite. Earth and Planetary Science Letters, 228: 125-142.

Gasser, D., Jeřábek, P., Faber, C., Stünitz, H., Menegon, L., Corfu, F., Erambert, M., et Whitehouse, M.J. 2015. Behaviour of geochronometers and timing of metamorphic reactions during deformation at lower crustal conditions: phase equilibrium modelling and $\mathrm{U}-\mathrm{Pb}$ dating of zircon, monazite, rutile and titanite from the Kalak Nappe Complex, northern Norway. Journal of Metamorphic Geology, 33: 513-534.

Geosoft. 2007. Oasis Montaj. Geosoft.

Gervais, F., et Brown, R.L. 2011. Testing modes of exhumation in collisional orogens: Synconvergent channel flow in the southeastern Canadian Cordillera. Lithosphere, 3: 55-75. doi:10.1130/L98.1.

Gervais, F., et Crowley, J.L. 2017. Prograde and near-peak zircon growth in a migmatitic pelitic schist of the southeastern Canadian Cordillera. Lithos, 282-283: 65-81. doi:10.1016/j.lithos.2017.02.016.

Gervais, F., et Hynes, A. 2013. Linking metamorphic textures to U-Pb monazite in-situ geochronology to determine the age and nature of aluminosilicate-forming reactions in the northern Monashee Mountains, British Columbia. Lithos, 160: 250-267. 
Gervais, F., Brown, R.L., et Crowley, J.L. 2010. Tectonic implications for a Cordilleran orogenic base in the Frenchman Cap dome, southeastern Canadian Cordillera. Journal of Structural Geology, 32: 941-959.

Gibson, H.D., Brown, R.L., et Carr, S.D. 2005. U-Th-Pb geochronologic constraints on the structural evolution of the Selkirk fan, northern Selkirk Mountains, southern Canadian Cordillera. Journal of Structural Geology, 27: 1899-1924.

Gower, C.F., et Krogh, T.E. 2002. A U-Pb geochronological review of the Proterozoic history of the eastern Grenville Province. Canadian Journal of Earth Sciences, 39: 795-829. doi:10.1139/e01-090.

Hanmer, S. 1988. Ductile thrusting at mid-crustal level, southwestern Grenville Province. Canadian Journal of Earth Sciences, 25: 1049-1059. doi:10.1139/e88-102.

Harley, S.L., Kelly, N.M., et Möller, A. 2007. Zircon behaviour and the thermal histories of mountain chains. Elements, 3: 25-30. doi:10.2113/gselements.3.1.25.

Hynes, A., et Rivers, T. 2010. Protracted continental collision - evidence from the Grenville Orogen. Canadian Journal of Earth Sciences, 47: 591-620. doi:10.1139/E10-003.

Hynes, A., Indares, A., Rivers, T., et Gobeil, A. 2000. Lithoprobe line 55: integration of out-ofplane seismic results with surface structure, metamorphism, and geochronology, and the tectonic evolution of the eastern Grenville Province. Canadian Journal of Earth Sciences, 37: 341-358. doi:10.1139/e99-076.

Hofmann, A.E., Baker, M.B., et Eiler, J.M. 2013. An experimental study of Ti and Zr partitioning among zircon, rutile, and granitic melt. 166: 235-253.

Indares, A., White, R.W., et Powell, R. 2008. Phase equilibria modelling of kyanite-bearing anatectic paragneisses from the central Grenville Province. Journal of Metamorphic Geology, 26: 815-836. doi:10.1111/j.1525-1314.2008.00788.x.

Indares, A., Dunning, G., Cox, R., Gale, D., et Connelly, J. 1998. High-pressure, hightemperature rocks from the base of thick continental crust: Geology and age constraints from the 
Manicouagan Imbricate Zone, eastern Grenville Province. Tectonics, 17: 426-440. doi:10.1029/98TC00373.

Jannin, S., Gervais, F., et Moukhsil, A. 2013. Trois phases de déformation autour de la zone de charriage de l'Allochtone au sud du réservoir Manicouagan (Grenville central, Québec). Ministère des Ressources Naturelles, Québec, MB 2013-06.

Jannin, S., Gervais, F., et Moukhsil, A. 2014. Les déformations permettant l'enfouissement et l'exhumation du Parautochtone au sud du réservoir Manicouagan (Province de Grenville central Québec). Ministère des Ressources Naturelles, Québec.

Jannin, S., Gervais, F., Moukhsil, A., Crowley, J.L., et Augland, L.E. Accepté pour publication. Datation $\mathrm{U} / \mathrm{Pb}$ des déformations inverses et normales dans la Province de Grenville central (Manicouagan, Québec): évidence d'un chenal orogénique dans le Parautochtone. In Géologie et ressources minérales de la partie centrale de la Province de Grenville. Moukhsil, A. coordonnateur. Ministère de l'Énergie et des Ressources naturelles, Québec, MM 2017 -.

Jiang, D. 2014. Structural geology meets micromechanics: A self-consistent model for the multiscale deformation and fabric development in Earth's ductile lithosphere. Journal of Structural Geology, 68: 247-272.

Jordan, S.L., Indares, A., et Dunning, G. 2006. Partial melting of metapelites in the Gagnon terrane below the high-pressure belt in the Manicouagan area (Grenville Province): pressuretemperature $(\mathrm{P}-\mathrm{T})$ and $\mathrm{U}-\mathrm{Pb}$ age constraints and implications. Canadian Journal of Earth Sciences, 43: 1309-1329. doi:10.1139/e06-038.

Kelsey, D.E., Clark, C., et Hand, M. 2008. Thermobarometric modelling of zircon and monazite growth in melt-bearing systems: Examples using model metapelitic and metapsammitic granulites. Journal of Metamorphic Geology, 26: 199-212.

Kretz, R. 1983. Symbols for rock-forming minerals. American Mineralogist, 68: 277-279.

Krogh, T.E., Kamo, S., Gower, C.F., et Owen, J.V. 2002. Augmented and reassessed U-Pb geochronological data from the Labradorian-Grenvillian front in the Smokey archipelago, eastern Labrador. Canadian Journal of Earth Sciences, 39: 831-843. doi:10.1139/e01-091. 
Ludwig, K.R. 2008. User's Manual for Isoplot 3.6. A Geochronological Toolkit for Microsoft Excel. Berkeley Geochronological Center, Special Publication, 4: 78.

Martel, E., van Breemen, O., Berman, R.G., et Pehrsson, S. 2008. Geochronology and tectonometamorphic history of the Snowbird Lake area, Northwest Territories, Canada: New insights into the architecture and significance of the Snowbird tectonic zone. Precambrian Research, 161: 201-230. doi:10.1016/j.precamres.2007.07.007.

Martignole, J., et Martelat, J.-E. 2005. Proterozoic mafic dykes as monitors of HP granulite facies metamorphism in the Grenville Front Tectonic Zone (western Quebec). Precambrian Research, 138: 183-207. doi:10.1016/j.precamres.2005.05.002.

Mehnert, K.R. 1968. Migmatites and the origin of granitic rocks. In Elsevier. Amsterdam.

Mezger, K., et Krogstad, E.J. 1997. Interpretation of discordant U-Pb zircon ages: an evaluation. Journal of metamorphic Geology, 15: 127-140.

Moukhsil, A., et Solgadi, F. Accepté pour publication. Géologie, potentiel minéral et cadre géodynamique des roches de la région du réservoir Daniel-Johnson (Manicouagan), partie centrale de la Province de Grenville, Québec. In Géologie et ressources minérales de la partie centrale de la Province de Grenville. Moukhsil, A. coordonnateur. Ministère de l'Énergie et des Ressources naturelles, Québec, MM 2017-.

Moukhsil, A., Solgadi, F., Clark, T., Blouin, S., Indares, A., et Davis, D.W. 2013. Géologie du nord-ouest de la région du barrage Daniel Johnson (Manic 5), Côte-Nord (22K14, 22K15, 22K16, 22N02 et 22N03). Ministère des Ressources Naturelles, Québec, RG 2013-01.

Owen, J.V., Rivers, T., et Gower, C.F. 1986. The Grenville front on the Labrador coast. In The Grenville Province, Geological Association of Canada, Special Paper. pp. 95-106.

Owen, J.V., Dallmeyer, R.D., Gower, C.F., et Rivers, T. 1988. Metamorphic conditions and ${ }^{40} \mathrm{Ar} /{ }^{39} \mathrm{Ar}$ geochronologic contracts across the Grenville Front zone, coastal Labrador, Canada. Lithos, 21: 13-35.

Parrish, R.R., et Noble, S.R. 2003. Zircon U-Th-Pb geochronology by Isotope Dilution Thermal Ionization Mass Spectrometry (ID-TIMS). Reviews in Mineralogy and Geochemistry, 53: 183-213. doi:10.2113/0530183. 
Passchier, C.W. 2001. Flanking structures. Journal of Structural geology, 23: 951-962.

Pyle, J.M., et Spear, F.S. 2003. Four generations of accessory-phase growth in low-pressure migmatites from SW New Hampshire. American Mineralogist, 88: 338-351.

Ramsay, J.G. 1967. Folding and fracturing of rocks. McGraw-Hill Book Cie, London.

Rivers, T. 1980. Revised stratigraphic nomenclature for Aphebian and other rock units, southern Labrador Trough, Grenville Province. Canadian Journal of Earth Sciences, 17: 668-670. doi:10.1139/e80-062.

Rivers, T. 1983a. The northern margin of the Grenville Province in western Labrador - anatomy of an ancient orogenic front. Precambrian Research, 22: 41-73. doi:10.1016/03019268(83)90058-X.

Rivers, T. 1983b. Progressive metamorphism of pelitic and quartzofeldspathic rocks in the Grenville Province of western Labrador - tectonic implications of bathozone 6 assemblages. Canadian Journal of Earth Sciences, 20: 1791-1804. doi:10.1139/e83-171.

Rivers, T. 1997. Lithotectonic elements of the Grenville Province: review and tectonic implications. Precambrian Research, 86: 117-154. doi:10.1016/S0301-9268(97)00038-7.

Rivers, T. 2008. Assembly and preservation of lower, mid, and upper orogenic crust in the Grenville Province-Implications for the evolution of large hot long-duration orogens. Precambrian Research, 167: 237-259. doi:10.1016/j.precamres.2008.08.005.

Rivers, T., et Corrigan, D. 2000. Convergent margin on southeastern Laurentia during the Mesoproterozoic: tectonic implications. Canadian Journal of Earth Sciences, 37: 359-383. doi:10.1139/e99-067.

Rivers, T., Martignole, J., Gower, C.F., et Davidson, A. 1989. New tectonic divisions of the Grenville Province, southeast Canadian Shield. Tectonics, 8: 63-84. doi:10.1029/TC008i001p00063.

Rivers, T., Culshaw, N., Hynes, A., Indares, A., Jamieson, R., et Martignole, J. 2012. The Grenville Orogen-A post-LITHOPROBE perspective. In Tectonic Styles in Canada: The LITHOPROBE Perspective. pp. 97-236. 
Rubatto, D. 2002. Zircon trace element geochemistry: partitioning with garnet and the link between U-Pb ages and metamorphism. Chemical Geology, 184: 123-138.

Rubatto, D., et Hermann, J. 2007. Experimental zircon/melt and zircon/garnet trace element partitioning and implications for the geochronology of crustal rocks. Chemical Geology, 241: 3861.

Rubatto, D., Williams, I.S., et Buick, I.S. 2001. Zircon and monazite response to prograde metamorphism in the Reynolds Range, central Australia. Contributions to Mineralogy and Petrology, 140: 458-468.

Rubatto, D., Hermann, J., et Buick, I.S. 2006. Temperature and bulk composition control on the growth of monazite and zircon during low-pressure anatexis (Mount Stafford, Central Australia). Journal of Petrology, 47: 1973-1996. doi:10.1093/petrology/eg1033.

Schärer, U., Krogh, T.E., et Gower, C.F. 1986. Age and evolution of the Grenville Province in eastern Labrador from U-Pb systematics in accessory minerals. Contributions to Mineralogy and Petrology, 94: 438-451.

Schmitz, M.D., et Schoene, B. 2007. Derivation of isotope ratios, errors, and error correlations for $\mathrm{U}-\mathrm{Pb}$ geochronology using ${ }^{205} \mathrm{~Pb}-{ }^{235} \mathrm{U}-\left({ }^{233} \mathrm{U}\right)$-spiked isotope dilution thermal ionization mass spectrometric data. Geochemistry, Geophysics, Geosystems, 8: Q08006. doi:10.1029/2006GC001492.

Simpson, C., et Schmid, S.M. 1983. An evaluation of criteria to deduce the sense of movement in sheared rocks. GSA Bulletin, 94: 1281-1288. doi:10.1130/00167606(1983)94\&lt;1281:AEOCTD\&gt;2.0.CO;2.

Smith, H.A., et Barreiro, B. 1990. Monazite U-Pb dating of staurolite grade metamorphism in pelitic schists. Contributions to Mineralogy and Petrology, 105: 602-615.

Soucy La Roche, R., Gervais, F., Tremblay, A., Crowley, J.L., et Ruffet, G. 2015. Tectonometamorphic history of the eastern Taureau shear zone, Mauricie area, Quebec: Implications for the exhumation of the mid-crust in the Grenville Province. Precambrian Research, 257: 22-46.

Spear, F.S., Kohn, M.J., et Cheney, J.T. 1999. P-T paths from anatectic pelites. 134: 17-32. 
Sun, S.-S., et McDonough, W. 1989. Chemical and isotopic systematics of oceanic basalts: implications for mantle composition and processes. Geological Society, London, Special Publications, 42: 313-345.

Thomson, S.D., Dickin, A.P., et Spray, J.G. 2011. Nd isotope mapping of Grenvillian crustal terranes in the vicinity of the Manicouagan Impact Structure. Precambrian Research, 191: 184 193. doi:10.1016/j.precamres.2011.08.006.

Tikoff, B., et Fossen, H. 1999. Three-dimensional reference deformations and strain facies. Journal of Structural Geology, 21: 1497-1512.

Vanderhaeghe, O., Teyssier, C., et Wysoczanski, R. 1999. Structural and geochronological constraints on the role of partial melting during the formation of the Shuswap metamorphic core complex at the latitude of the Thor-Odin dome, British Columbia. Canadian Journal of Earth Sciences, 36: 917-943. doi:10.1139/e99-023.

van Gool, J., Rivers, T., et Calon, T. 2008. Grenville Front zone, Gagnon terrane, southwestern Labrador: Configuration of a midcrustal foreland fold-thrust belt. Tectonics, 27: TC1004. doi:10.1029/2006TC002095.

Vavra, G. 1990. On the kinematics of zircon growth and its petrogenetic significance: a cathodoluminescence study. Contributions to Mineralogy and Petrology, 106: 90-99.

Vavra, G., Schmid, R., et Gebauer, D. 1999. Internal morphology, habit and U-Th-Pb microanalysis of amphibolite-to-granulite facies zircons: geochronology of the Ivrea Zone (Southern Alps). Contributions to Mineralogy and Petrology, 134: 380-404. doi:10.1007/s004100050492.

Vernon, R.H., Etheridge, M.A., et Wall, V.J. 1988. Shape and microstructure of microgranitoid enclaves: indicators of magma mingling and flow. Lithos, 22: 1-11.

Waters, D.J. 2001. The significance of prograde et retrograde quartz-bearing intergrowth microstructures in partially melted granulite-facies rocks. Lithos, 56: 97-110. doi:10.1016/S0024-4937(00)00061-X.

Williams, P.F. 1985. Multiply deformed terrains - problems of correlation. Journal of Structural Geology, 7: 269-280. 
Williams, M.L., et Jercinovic, M.J. 2012. Tectonic interpretation of metamorphic tectonites: integrating compositional mapping, microstructural analysis and in situ monazite dating. Journal of Metamorphic Geology, 30: 739-752. doi:10.1111/j.1525-1314.2012.00995.x.

Yakymchuk, C., et Brown, M. 2014. Behaviour of zircon and monazite during crustal melting. Journal of the Geological Society, 171: 465-479. 
Tableau 1 : Sommaire des fabriques de toutes les déformations dans la Ceinture parautochtone de la région d'étude (modifié de Jannin et al., accepté pour publication).

\begin{tabular}{|c|c|c|c|c|c|c|c|}
\hline $\begin{array}{l}\text { Défor } \\
\text { mation }\end{array}$ & Secteur & Foliation & Linéation & $\begin{array}{l}\text { Intensité } \\
\text { déformation }\end{array}$ & $\begin{array}{l}\text { Plan } \\
\text { axial }\end{array}$ & Axe de pli & Cisaillement \\
\hline \multirow[t]{3}{*}{ D1 } & $\mathrm{A}$ & N110/75 & modérée $\rightarrow \mathrm{SSE}$ & & & & \\
\hline & B & N101/68; & $38 \rightarrow \mathrm{N} 138$ & & & & \\
\hline & $\mathrm{F}$ & $\mathrm{N} 110 / 73$ & $37 \rightarrow \mathrm{N} 122$ & $3-4$ & & $30 \rightarrow \mathrm{N} 111$ & $\begin{array}{l}\text { N098/64 dextre et N140/53 } \\
\text { inverse-dextre }\end{array}$ \\
\hline D1? & $\mathrm{C}$ & $\mathrm{O}$ à N/modérée & $\begin{array}{l}\text { modérée } \longrightarrow \text { N000 } \\
\text { à N150 }\end{array}$ & 4 & & modéré $\rightarrow \mathrm{SE}$ & $\begin{array}{l}\text { N127/82 inverse, N330/67 } \\
\text { dextre et senestre, N337/56 } \\
\text { senestre }\end{array}$ \\
\hline \multirow[t]{4}{*}{ D2 } & B & $\begin{array}{l}\text { schistosité de plan axial; } \\
\text { foliation N048/52 }\end{array}$ & $28 \rightarrow 201$ & & N050/84 & $\begin{array}{l}\text { variable, fort } \\
\text { plongement }\end{array}$ & apparent senestre \\
\hline & $\mathrm{D}$ & $\begin{array}{l}\text { N029/65 (pendage de } 20 \\
\left.\text { à } 90^{\circ}\right) ; \text { N028/60 }\end{array}$ & $\begin{array}{l}\mathbf{2 1} \rightarrow \mathbf{N 1 8 9} \\
28 \rightarrow \mathrm{N} 193\end{array}$ & variable : $3-5$ & & $\begin{array}{l}\mathbf{1 9} \rightarrow \mathbf{N} 195 \\
21 \rightarrow \mathrm{N} 189\end{array}$ & senestre et inverse \\
\hline & D ouest & $\begin{array}{l}\text { Plissée et répartie sur un } \\
\text { plan N280/75 }\end{array}$ & & & & $15 \rightarrow \mathrm{N} 190$ & inverse-décrochant \\
\hline & E & N067/65; N065/58 & modérée $\rightarrow \mathrm{N} 170$ & $\begin{array}{l}\text { pli : charnière } \\
\text { 2-3, flanc } 4-5\end{array}$ & & & \\
\hline D2? & $\mathrm{C}$ & NNO à N/80 & $\mathrm{L} 2 ?: \sim 20 \rightarrow \mathrm{S}$ & $\bar{c}$ & & & N181/79 senestre \\
\hline $\begin{array}{l}\mathrm{D} 2 \\
\text { tardive }\end{array}$ & $\mathrm{D}$ & $\begin{array}{l}\text { plan de cisaillement } \\
\text { schistosité de plan axial }\end{array}$ & & & N029/75 & & $\begin{array}{l}\text { N055/82 senestre-normal } \\
\text { dextre et senestre }\end{array}$ \\
\hline D3 & $\mathrm{E}$ & N070/42 & $13 \rightarrow \mathrm{N} 239$ & 4 & & $\mathbf{3 1 \rightarrow N 2 3 7}$ & dextre-normal \\
\hline
\end{tabular}


Tableau 2 : Concentrations normalisées aux chondrites des ÉTR calculés pour les grains de zircon des échantillons 12SJ14 et 12SJ27E par

\section{LA-ICP-MS.}

\begin{tabular}{|c|c|c|c|c|c|c|c|c|c|c|c|c|c|c|c|c|}
\hline \multirow{2}{*}{$\begin{array}{l}\text { Groupe } \\
\text { CL }\end{array}$} & \multirow{2}{*}{$\begin{array}{c}\text { Groupe } \\
\text { ÉTR } \\
\text { (Fig. } 9 \\
\text { et 10) }\end{array}$} & \multirow{2}{*}{$\begin{array}{c}\mathrm{N}^{\circ} \\
\mathrm{d}^{\prime} \text { Analyse }\end{array}$} & \multicolumn{14}{|c|}{ Concentrations normalisées aux valeurs des chondrites de Sun et McDonough (1989) } \\
\hline & & & $\mathrm{La}$ & $\mathrm{Ce}$ & $\operatorname{Pr}$ & $\mathrm{Nd}$ & $\mathrm{Sm}$ & $\mathrm{Eu}$ & $\mathrm{Gd}$ & $\mathrm{Tb}$ & Dy & Ho & Er & $\mathrm{Tm}$ & $\mathrm{Yb}$ & $\mathrm{Lu}$ \\
\hline \multicolumn{17}{|c|}{ Echantillon 12SJ14; analyses LA-ICP-MS } \\
\hline \multirow{17}{*}{$\begin{array}{l}\text { No } \\
0 \\
\vdots \\
\vdots \\
\vdots \\
0\end{array}$} & \multirow{12}{*}{ 9D3 } & 43 & & 0,9141 & 0,0805 & 0,3603 & 1,6285 & 7,3140 & 19,0094 & 40,0712 & 47,1660 & 44,1416 & 34,8171 & 46,8868 & 49,5668 & 27,1769 \\
\hline & & 44 & & 0,9435 & & 0,3065 & 2,9932 & 8,7380 & 20,2644 & 48,7436 & 73,3149 & 79,4533 & 76,3100 & 95,6939 & 127,3563 & 82,3809 \\
\hline & & 45 & 0,1017 & 1,3831 & 0,1819 & 0,8141 & 2,9471 & 4,4874 & 19,7443 & 28,5383 & 32,1601 & 29,3092 & 21,8604 & 21,3354 & 31,2207 & 18,6437 \\
\hline & & 46 & & 1,1129 & & 0,2480 & 2,4222 & 9,8752 & 30,0753 & 59,3894 & 71,0574 & 62,2812 & 53,7721 & 61,0735 & 69,1149 & 37,9185 \\
\hline & & 49 & & 1,0626 & & 0,3365 & 3,2866 & 10,5759 & 22,1629 & 39,7312 & 52,3119 & 49,7328 & 40,1851 & 42,3227 & 51,3413 & 30,0207 \\
\hline & & 55 & 0,0035 & 0,9904 & 0,1403 & 0,2768 & 5,0219 & 5,2539 & 13,6403 & 31,3921 & 35,2031 & 27,0568 & 25,9302 & 23,6871 & 26,6954 & 17,4631 \\
\hline & & 58 & & 1,2261 & & 0,2986 & 2,9161 & 7,8799 & 25,3554 & 49,4462 & 63,0067 & 71,6889 & 53,0530 & 62,2216 & 87,3587 & 51,4955 \\
\hline & & 59 & 0,2241 & 1,7557 & 0,2156 & 1,6204 & 6,1480 & 4,4195 & 18,7062 & 30,7837 & 42,9415 & 46,9348 & 39,0295 & 43,2659 & 48,6752 & 29,4516 \\
\hline & & 66 & & 1,0751 & & 0,1382 & 1,3498 & 4,2923 & 13,6304 & 35,9868 & 46,2662 & 44,8606 & 34,6998 & 40,5900 & 45,2946 & 34,7003 \\
\hline & & 68 & & 1,2277 & & 0,3368 & 3,2896 & 8,3634 & 29,5053 & 63,3344 & 94,0992 & 91,4125 & 84,0649 & 83,7939 & 96,1625 & 69,5234 \\
\hline & & 70 & & 1,0410 & 0,0652 & 0,2919 & 1,8065 & 2,5859 & 13,4419 & 39,5702 & 63,4612 & 68,4077 & 66,0543 & 80,5359 & 94,2174 & 81,9780 \\
\hline & & 73. & & 0,7545 & & 0,3757 & _ & 8,3138 & 29,0251 & 61,7180 & 99,8584 & 124,3727 & 143,8138 & 161,3072 & 205,3518 & 172,7485 \\
\hline & \multirow{8}{*}{ 9D4 } & 38 & 0,0122 & 1,2351 & 0,4860 & 0,4972 & 3,8986 & 6,5428 & 16,9500 & 39,1309 & 43,8707 & 48,2704 & 43,0218 & 40,9169 & 57,2133 & 28,2387 \\
\hline & & 40 & 0,1431 & 0,9326 & 0,4179 & 0,5095 & 3,6911 & 3,9012 & 14,6080 & 35,7207 & 54,7104 & 66,9500 & 75,8385 & 87,7952 & 127,6354 & 75,7519 \\
\hline & & 41 & 12,5993 & 14,7523 & 22,1986 & 23,9201 & 42,0919 & 20,6726 & 51,6065 & 65,5901 & 68,2840 & 64,4061 & 53,0012 & 45,4057 & 59,2109 & 34,1358 \\
\hline & & 56 & 3,5690 & 8,8167 & 8,8561 & 17,2385 & 58,9365 & 43,1482 & 104,0571 & 86,2403 & 88,0528 & 61,6705 & 42,3076 & 39,0065 & 44,0739 & 22,0092 \\
\hline & & 71 & 0,3615 & 2,3587 & 1,0553 & 2,2679 & 7,3121 & 13,9267 & 36,8013 & 62,9810 & 78,1009 & 71,1126 & 62,4872 & 70,2160 & 60,8479 & 51,7052 \\
\hline \multirow{9}{*}{$\begin{array}{l}\overrightarrow{0} \\
\stackrel{0}{0} \\
\text { o. }\end{array}$} & & 67 & 4,4338 & 9,9344 & 10,0722 & 14,9732 & 43,7566 & 30,6374 & 76,8869 & 98,8515 & 85,3894 & 71,0616 & 52,7467 & 52,2471 & 51,1513 & 37,6000 \\
\hline & & 69 & 0,8357 & 4,0621 & 1,9059 & 3,6196 & 23,1456 & 21,1993 & 49,8381 & 78,1413 & 97,0772 & 91,2941 & 78,1368 & 71,7293 & 79,7445 & 56,4024 \\
\hline & & 74 & 2,5503 & 7,2039 & 7,3777 & 10,2583 & ـ & 27,1202 & 70,3300 & 93,3524 & 93,2971 & ـ & 85,5768 & 102,6416 & 118,8482 & 82,0149 \\
\hline & \multirow{6}{*}{ 9D2 } & 39 & & 1,4603 & & 0,6088 & 5,9462 & 13,1688 & 33,9252 & 53,1700 & 59,4703 & 52,1628 & 43,0546 & 44,2827 & 53,1999 & 30,1714 \\
\hline & & 47 & & 1,2870 & 0,0868 & 0,3885 & 6,7743 & 11,0793 & 25,4734 & 44,5021 & 45,2766 & 42,2431 & 38,8846 & 38,7232 & 43,8567 & 24,4300 \\
\hline & & 48 & & 1,1400 & & 0,0730 & 0,7127 & 4,1491 & 18,6569 & 27,5291 & 39,6555 & 38,0422 & 28,0191 & 26,1275 & 32,7412 & 18,9038 \\
\hline & & 50 & & 1,8105 & & 0,5389 & 5,2634 & 8,8182 & 28,4432 & 37,8156 & 47,1764 & 45,9521 & 36,3835 & 29,8383 & 41,5505 & 22,3381 \\
\hline & & 52 & & 1,9711 & 0,1093 & 0,4892 & 9,1791 & 22,6702 & 48,5591 & 93,9763 & 107,4653 & 115,4850 & 100,6051 & 112,8327 & 140,1475 & 91,6310 \\
\hline & & 57 & & 1,1593 & & 0,1696 & 1,6570 & 8,3162 & 13,5067 & 33,2046 & 34,1094 & 28,9698 & 25,1762 & 26,1149 & 33,1747 & 17,2167 \\
\hline
\end{tabular}




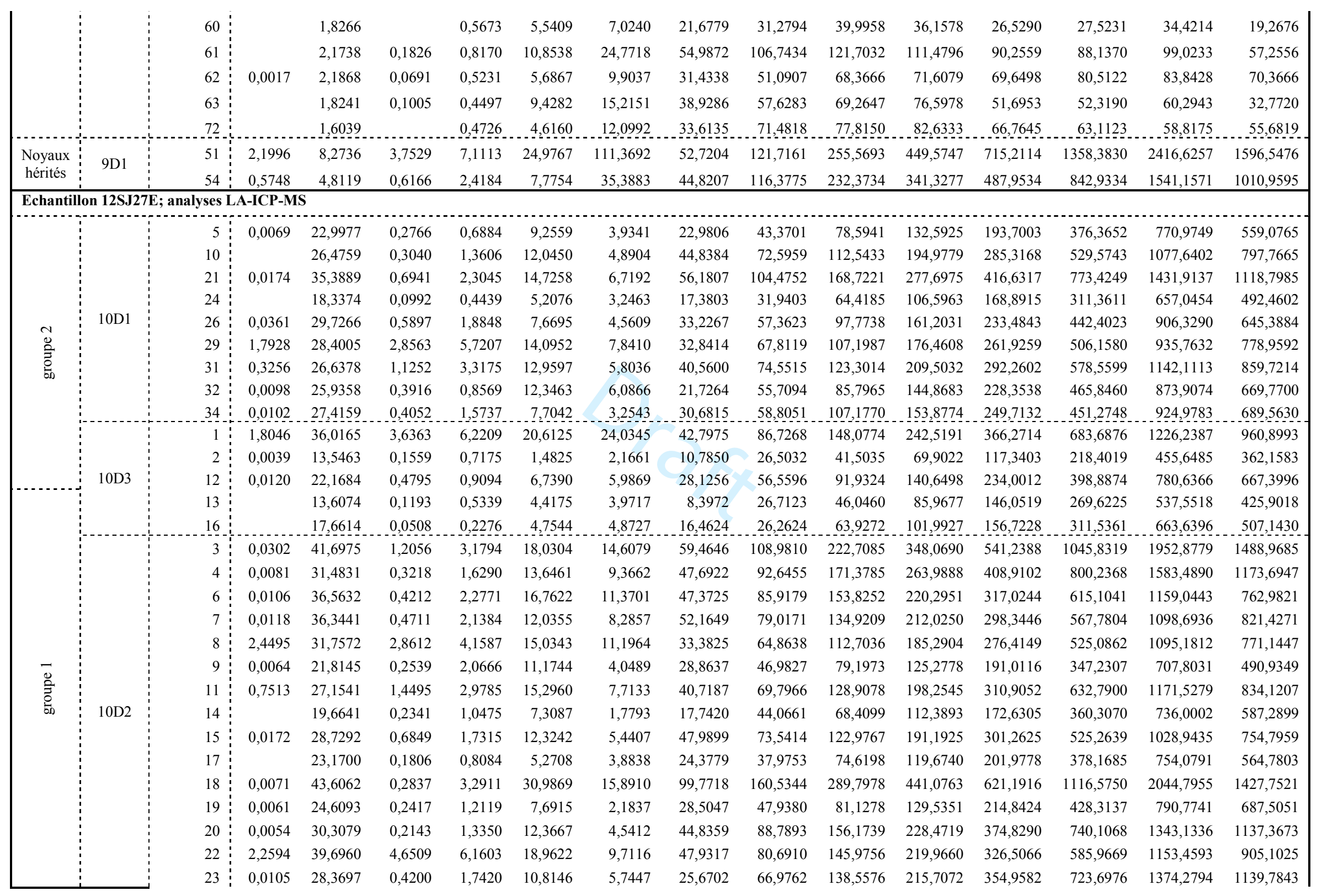




\begin{tabular}{r:rrrrrrrrrrrrrr}
25 & 0,0147 & 49,5921 & 0,5882 & 5,2328 & 29,5144 & 18,7718 & 94,9323 & 177,6723 & 296,2762 & 427,0193 & 662,1160 & 1130,2860 & 2093,4670 & 1406,9149 \\
28 & 0,0377 & 53,9532 & 1,5051 & 7,3389 & 49,2441 & 30,7872 & 159,9099 & 255,5955 & 406,1217 & 613,3260 & 789,0787 & 1393,4504 & 2388,3014 & 1809,0701 \\
30 & 0,0085 & 26,5763 & 0,3402 & 1,0887 & 10,0913 & 3,9706 & 39,0801 & 56,0995 & 112,3448 & 179,1224 & 288,6587 & 596,7659 & 1184,3430 & 909,4405 \\
33 & 0,0563 & 18,8613 & 0,1811 & 0,8688 & 9,0711 & 8,0227 & 25,8486 & 42,0815 & 87,7665 & 139,8029 & 227,6874 & 417,9082 & 826,0934 & 628,5659 \\
\hline
\end{tabular}


Tableau 3 : Températures calculées en utilisant le thermomètre de Ti dans le zircon (Hofmann et al., 2013) avec une pression estimée à $1300 \mathrm{MPa}(\Delta \mathrm{P}=200 \mathrm{MPa}$; Jordan et al., 2006) pour les grains de zircon des groupes 1 et 2 de l'échantillon 12SJ14 par LA-ICP-MS $\left(\mathrm{aTiO}_{2}=1 ; \mathrm{aSiO}_{2}=\right.$ $1)$.

\begin{tabular}{|c|c|c|c|c|c|}
\hline $\begin{array}{l}\text { Groupe } \\
\text { CL }\end{array}$ & $\begin{array}{c}\text { Groupe ÉTR } \\
\text { (Fig. 9) }\end{array}$ & $\begin{array}{c}\mathrm{N}^{\circ} \\
\text { d'Analyse }^{2}\end{array}$ & $\mathrm{Ti}(\mathrm{ppm})$ & $\mathrm{T}\left({ }^{\circ} \mathrm{C}\right)$ & $\Delta \mathrm{T}\left({ }^{\circ} \mathrm{C}\right)$ \\
\hline \multicolumn{6}{|c|}{ Echantillon 12SJ14; analyses LA-ICP-MS } \\
\hline \multirow{17}{*}{$\begin{array}{l}\mathcal{N} \\
\mathscr{O} \\
\stackrel{\Xi}{0} \\
\text { b. }\end{array}$} & \multirow{12}{*}{ 9D3 } & 43 & 2,2184129 & 716 & 30 \\
\hline & & 44 & 2,340093 & 720 & 30 \\
\hline & & 45 & 2,0919205 & 711 & 29 \\
\hline & & 46 & 2,7427032 & 732 & 30 \\
\hline & & 49 & 2,6215854 & 728 & 30 \\
\hline & & 55 & 1,8549434 & 703 & 29 \\
\hline & & 58 & 2,3720393 & 721 & 30 \\
\hline & & 59 & 1,9405162 & 706 & 29 \\
\hline & & 66 & 1,8609522 & 703 & 29 \\
\hline & & 68 & 2,0585623 & 710 & 29 \\
\hline & & 70 & 2,5947334 & 727 & 30 \\
\hline & & 73 & 2,958743 & 737 & 31 \\
\hline & \multirow{8}{*}{ 9D4 } & 38 & 2,382855 & 721 & 30 \\
\hline & & 40 & 1,9075508 & 705 & 29 \\
\hline & & 71 & 3,0052502 & 738 & 31 \\
\hline & & 41 & 3,9314014 & 759 & 32 \\
\hline & & 56 & 3,8865863 & 759 & 32 \\
\hline \multirow{14}{*}{$\begin{array}{l}\overrightarrow{0} \\
\stackrel{0}{0} \\
\text { b. }\end{array}$} & & 67 & 4,2025948 & 765 & 32 \\
\hline & & 69 & 4,1327885 & 764 & 32 \\
\hline & & 74 & 3,3144046 & 746 & 31 \\
\hline & \multirow{11}{*}{ 9D2 } & 39 & 2,1462761 & 713 & 30 \\
\hline & & 47 & 2,0079413 & 708 & 29 \\
\hline & & 48 & 2,15121 & 713 & 30 \\
\hline & & 50 & 1,9816589 & 708 & 29 \\
\hline & & 52 & 2,4815608 & 724 & 30 \\
\hline & & 57 & 1,3635094 & 681 & 28 \\
\hline & & 60 & 2,1875678 & 715 & 30 \\
\hline & & 61 & 3,1656298 & 742 & 31 \\
\hline & & 62 & 2,1170697 & 712 & 30 \\
\hline & & 63 & 2,8167725 & 734 & 31 \\
\hline & & 72 & 2,6120266 & 728 & 30 \\
\hline
\end{tabular}


Tableau 4 : Ratios des divers isotopes uranium et plomb avec les âges isotopiques calculés pour les grains de zircon des échantillons $12 \mathrm{SJ} 14$ et 12SJ27E par LA-ICP-MS (en gras = dates utilisées pour calculer les âges des phases de déformation; modifié de Jannin et al., 2014, accepté pour publication).

\begin{tabular}{|c|c|c|c|c|c|c|c|c|c|c|c|c|c|c|c|c|c|c|}
\hline $\begin{array}{l}\text { Grou } \\
\text { pe } \\
\text { CL }\end{array}$ & $\begin{array}{c}\text { Groupe } \\
\text { ÉTR } \\
\text { (Fig. } 9 \\
\text { et 10) }\end{array}$ & $\begin{array}{c}\mathrm{N}^{\circ} \\
\mathrm{d}^{\prime} \text { Anal } \\
\text { yse }\end{array}$ & $\begin{array}{l}\text { Conce } \\
\text { U } \\
(\mathrm{ppm} \\
)^{2}\end{array}$ & $\begin{array}{l}\text { tration } \\
\mathrm{Pb}^{*} \\
(\mathrm{ppm})\end{array}$ & $\begin{array}{c}\mathrm{Th} / \\
\mathrm{U}\end{array}$ & ${ }^{207} \mathrm{~Pb} /{ }^{206} \mathrm{~Pb}$ & $\begin{array}{l} \pm 2 \sigma \\
(\%)\end{array}$ & ${ }^{207} \mathrm{~Pb} /{ }^{235} \mathrm{U}$ & $\begin{array}{l} \pm 2 \sigma \\
(\%)\end{array}$ & ${ }^{206} \mathrm{~Pb} /{ }^{238} \mathrm{U}$ & $\begin{array}{l} \pm 2 \sigma \\
(\%)\end{array}$ & rho & $\begin{array}{l}\text { Age (Ma) } \\
{ }^{207} \mathrm{~Pb} /{ }^{206} \mathrm{~Pb}\end{array}$ & $\begin{array}{l} \pm 2 \sigma \\
(\mathrm{Ma})\end{array}$ & $\begin{array}{l}\text { Age } \\
(\mathrm{Ma}) \\
{ }^{207} \mathrm{~Pb} /{ }^{2} \\
{ }^{35} \mathrm{U}\end{array}$ & $\begin{array}{l} \pm 2 \sigma \\
(\mathrm{Ma})\end{array}$ & $\begin{array}{l}\text { Age (Ma) } \\
{ }^{206} \mathrm{~Pb}{ }^{238} \mathrm{U}\end{array}$ & $\begin{array}{l} \pm 2 \sigma \\
\text { (Ma) }\end{array}$ \\
\hline \multicolumn{19}{|c|}{ Echantillon 12SJ14; analyses LA-ICP-MS } \\
\hline \multirow{17}{*}{ 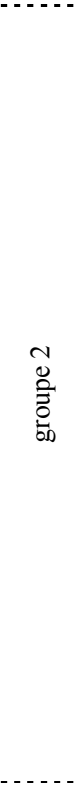 } & \multirow{12}{*}{ 9D3 } & 43 & 222 & 38,7 & 0,01 & 13,7989 & 3,1514 & 1,6096 & 6,1948 & 0,1611 & 5,3333 & 0,86 & 999 & 64 & 974 & 39 & 963 & 48 \\
\hline & & 44 & 240 & 42,0 & 0,02 & 13,3995 & 4,3304 & 1,6644 & 9,1712 & 0,1618 & 8,0845 & 0,88 & 1059 & 87 & 995 & 58 & 967 & 73 \\
\hline & & 45 & 314 & 57,5 & 0,02 & 14,0248 & 4,1755 & 1,6682 & 6,2729 & 0,1697 & 4,6812 & 0,75 & 966 & 85 & 996 & 40 & 1010 & 44 \\
\hline & & 46 & 240 & 42,7 & 0,02 & 14,21 & 4,1454 & 1,5982 & 6,8702 & 0,1647 & 5,4787 & 0,8 & 939 & 85 & 970 & 43 & 983 & 50 \\
\hline & & 49 & 245 & 43,6 & 0,02 & 14,0783 & 3,6051 & 1,6138 & 6,4659 & 0,1648 & 5,3676 & 0,83 & 958 & 74 & 976 & 41 & 983 & 49 \\
\hline & & 55 & 291 & 50,6 & 0,01 & 13,5579 & 4,1335 & 1,6344 & 6,4904 & 0,1607 & 5,0039 & 0,77 & 1035 & 84 & 984 & 41 & 961 & 45 \\
\hline & & 58 & 188 & 33,0 & 0,02 & 13,7604 & 3,347 & 1,6314 & 6,7846 & 0,1628 & 5,9012 & 0,87 & 1005 & 68 & 982 & 43 & 972 & 53 \\
\hline & & 59 & 199 & 35,2 & 0,03 & 14,0073 & 3,7222 & 1,6128 & 5,8367 & 0,1638 & 4,4958 & 0,77 & 969 & 76 & 975 & 37 & 978 & 41 \\
\hline & & 66 & 191 & 34,4 & 0,02 & 13,9205 & 2,0362 & 1,6541 & 3,0539 & 0,167 & 2,2759 & 0,75 & 981 & 41 & 991 & 19 & 996 & 21 \\
\hline & & 68 & 163 & 28,8 & 0,03 & 13,7429 & 1,5351 & 1,6375 & 2,8861 & 0,1632 & 2,444 & 0,85 & 1007 & 31 & 985 & 18 & 975 & 22 \\
\hline & & 70 & 152 & 26,1 & 0,02 & 13,6411 & 2,3414 & 1,6121 & 4,2765 & 0,1595 & 3,5786 & 0,84 & 1022 & 47 & 975 & 27 & 954 & 32 \\
\hline & & 73 & 154 & 27,5 & 0,02 & 13,9549 & 2,4305 & 1,6408 & 4,6286 & 0,1661 & 3,9391 & 0,85 & 976 & 50 & 986 & 29 & 990. & 36 \\
\hline & \multirow{8}{*}{ 9D4 } & 38 & 190 & 34,9 & 0,02 & 13,3825 & 2,7457 & 1,7024 & 5,0748 & 0,1652 & 4,2679 & 0,84 & 1061 & 55 & 1009 & 32 & 986 & 39 \\
\hline & & 40 & 173 & 31,0 & 0,02 & 13,8145 & 3,1005 & 1,6562 & 6,547 & 0,1659 & 5,7662 & 0,88 & 997 & 63 & 992 & 41 & 990 & 53 \\
\hline & & 41 & 250 & 43,4 & 0,04 & 13,8946 & 3,3202 & 1,5761 & 5,7789 & 0,1588 & 4,7299 & 0,82 & 985 & 68 & 961 & 36 & 950 & 42 \\
\hline & & 56 & 335 & 62,2 & 0,05 & 13,3841 & 2,8735 & 1,7638 & 5,5839 & 0,1712 & 4,7878 & 0,86 & 1061 & 58 & 1032 & 36 & 1019 & 45 \\
\hline & & 71 & 174 & 30,5 & 0,05 & 13,6521 & 3,0967 & 1,6402 & 4,6134 & 0,1624 & 3,4196 & 0,74 & 1021 & 63 & 986 & 29 & 970 & 31 \\
\hline \multirow{7}{*}{$\begin{array}{l}\overrightarrow{0} \\
\stackrel{0}{0} \\
\text { b. }\end{array}$} & & 67 & 368 & 65,3 & 0,06 & 13,6612 & 1,7832 & 1,6567 & 3,0292 & 0,1641 & 2,4487 & 0,81 & 1019 & 36 & 992 & 19 & 980 & 22 \\
\hline & & 69 & 436 & 75,6 & 0,04 & 13,6529 & 1,6363 & 1,6133 & 2,8039 & 0,1597 & 2,277 & 0,81 & 1021 & 33 & 975 & 18 & 955 & 20 \\
\hline & & 74 & 648 & 116,2 & 0,03 & 13,6629 & 1,0408 & 1,6744 & 4,2105 & 0,1659 & 4,0798 & 0,97 & 1019 & 21 & 999 & 27 & 990 & 37 \\
\hline & \multirow{4}{*}{ 9D2 } & 39 & 428 & 77,2 & 0,02 & 13,8921 & 1,6639 & 1,6613 & 3,0796 & 0,1674 & 2,5915 & 0,84 & 985 & 34 & 994 & 20 & 998 & 24 \\
\hline & & 47 & 361 & 64,3 & 0,02 & 13,7336 & 2,6737 & 1,6576 & 5,1967 & 0,1651 & 4,4562 & 0,86 & 1009 & 54 & 992 & 33 & 985 & 41 \\
\hline & & 48 & 334 & 58,2 & 0,01 & 13,8746 & 3,1183 & 1,6063 & 6,1689 & 0,1616 & 5,3228 & 0,86 & 988 & 63 & 973 & 39 & 966 & 48 \\
\hline & & 50 & 413 & 69,8 & 0,02 & 13,7634 & 1,6481 & 1,5699 & 4,305 & 0,1567 & 3,977 & 0,92 & 1004 & 33 & 958 & 27 & 938 & 35 \\
\hline
\end{tabular}




\begin{tabular}{|c|c|c|c|c|c|c|c|c|c|c|c|c|c|c|c|c|c|c|}
\hline & & 52 & 706 & 122,1 & 0,02 & 13,6926 & 1,483 & 1,6138 & 4,4188 & 0,1603 & 4,1625 & 0,94 & 1015 & 30 & 976 & 28 & 958 & 37 \\
\hline & & 57 & 263 & 47,1 & 0,02 & 14,0312 & 2,1936 & 1,631 & 5,2836 & 0,166 & 4,8068 & 0,91 & 965 & 45 & 982 & 33 & 990 & 44 \\
\hline & & 60 & 401 & 71,0 & 0,02 & 13,8212 & 2,343 & 1,6383 & 4,2693 & 0,1642 & 3,5689 & 0,84 & 996 & 48 & 985 & 27 & 980 & 32 \\
\hline & & 61 & 559 & 100,0 & 0,02 & 13,4564 & 2,3904 & 1,6727 & 4,4642 & 0,1633 & 3,7703 & 0,84 & 1050 & 48 & 998 & 28 & 975 & 34 \\
\hline & & 62 & 631 & 115,8 & 0,02 & 13,3886 & 1,823 & 1,7263 & 3,6538 & 0,1676 & 3,1665 & 0,87 & 1060 & 37 & 1018 & 23 & 999 & 29 \\
\hline & & 63 & 346 & 60,1 & 0,04 & 13,8151 & 1,7279 & 1,6014 & 4,909 & 0,1605 & 4,5949 & 0,94 & 997 & 35 & 971 & 31 & 959 & 41 \\
\hline & & 72 & 264 & 48,1 & 0,02 & 13,6958 & 2,1992 & 1,6981 & 4,0728 & 0,1687 & 3,428 & 0,84 & 1014 & 45 & 1008 & 26 & 1005 & 32 \\
\hline $\begin{array}{c}\text { Noya } \\
\text { ux }\end{array}$ & \multirow{2}{*}{ 9D1 } & 51 & 803 & 365,8 & 0,04 & 6,1269 & 1,9442 & 8,694 & 3,5507 & 0,3863 & 2,9712 & 0,84 & 2489 & 33 & 2306 & 32 & 2106 & 53 \\
\hline $\begin{array}{c}\text { hérit } \\
\text { és }\end{array}$ & & 54 & 831 & 397,3 & 0,04 & 6,4543 & 3,441 & 8,7262 & 8,2144 & 0,4085 & 7,459 & 0,91 & 2401 & 59 & 2310 & 75 & 2208 & 139 \\
\hline \multicolumn{19}{|c|}{ Echantillon 12SJ27E; analyses LA-ICP-MS } \\
\hline \multirow{12}{*}{ 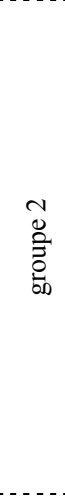 } & \multirow{9}{*}{ 10D1 } & 5 & 133 & 28,1 & 0,73 & 14,3443 & 2,8509 & 1,5229 & 3,7574 & 0,1584 & 2,4475 & 0,65 & 920 & 59 & 940 & 23 & 948 & 22 \\
\hline & & 10 & 155 & 35,0 & 0,82 & 13,8854 & 3,1522 & 1,6108 & 4,8635 & 0,1622 & 3,7037 & 0,76 & 986 & 64 & 974 & 30 & 969 & 33 \\
\hline & & 21 & 192 & 43,4 & 1,10 & 14,0051 & 2,6025 & 1,5594 & 4,7895 & 0,1584 & 4,0208 & 0,84 & 969 & 53 & 954 & 30 & 948 & 35 \\
\hline & & 24 & 147 & 31,2 & 0,69 & 14,2157 & 2,949 & 1,5646 & 4,8121 & 0,1613 & 3,8026 & 0,79 & 938 & 60 & 956 & 30 & 964 & 34 \\
\hline & & 26 & 186 & 40,5 & 0,79 & 13,7685 & 2,7243 & 1,5882 & 5,0145 & 0,1586 & 4,21 & 0,84 & 1004 & 55 & 966 & 31 & 949 & 37 \\
\hline & & 29 & 163 & 35,4 & 0,95 & 13,8512 & 2,5468 & 1,5677 & 4,2855 & 0,1575 & 3,4467 & 0,8 & 991 & 52 & 957 & 27 & 943 & 30 \\
\hline & & 31 & 124 & 27,9 & 1,01 & 14,3436 & 2,717 & 1,5465 & 4,0748 & 0,1609 & 3,0367 & 0,75 & 920 & 56 & 949 & 25 & 962 & 27 \\
\hline & & 32 & 149 & 32,9 & 0,82 & 14,1263 & 2,4497 & 1,5842 & 3,715 & 0,1623 & 2,7929 & 0,75 & 951 & 50 & 964 & 23 & 970 & 25 \\
\hline & & 34 & 181 & 40,1 & 0,78 & 14,0241 & 1,7547 & 1,6106 & 3,7256 & 0,1638 & 3,2866 & 0,88 & 966 & 36 & 974 & 23 & 978 & 30 \\
\hline & \multirow{18}{*}{$10 \mathrm{D} 2$} & 1 & 147 & 33,9 & 1,17 & 13,8075 & 1,8615 & 1,5875 & 3,8309 & 0,159 & 3,3482 & 0,87 & 998 & 38 & 965 & 24 & 951 & 30 \\
\hline & & 2 & 242 & 47,4 & 0,42 & 13,759 & 2,6674 & 1,6234 & 4,7426 & 0,162 & 3,9214 & 0,83 & 1005 & 54 & 979 & 30 & 968 & 35 \\
\hline & & 12 & 105 & 23,3 & 1,09 & 13,9899 & 3,3465 & 1,5653 & 5,2637 & 0,1588 & 4,063 & 0,77 & 971 & 68 & 957 & 33 & 950 & 36 \\
\hline \multirow{15}{*}{$\begin{array}{l}\overrightarrow{0} \\
\stackrel{0}{0} \\
\stackrel{0}{0}\end{array}$} & & 13 & 178 & 34,4 & 0,49 & 14,0357 & 2,0981 & 1,5479 & 4,0682 & 0,1576 & 3,4854 & 0,86 & 964 & 43 & 950 & 25 & 943 & 31 \\
\hline & & 16 & 168 & 34,9 & . & 14,0724 & . & 1,5591 & 4,6261 & 0,1591 & 3,4589 & 0,75 & 959 & 63 & 954 & 29. & 952 & 31. \\
\hline & & 3 & 506 & 114,2 & 0,87 & 13,7572 & 2,2808 & 1,6542 & 3,7671 & 0,1651 & 2,9982 & 0,8 & 1005 & 46 & 991 & 24 & 985 & 27 \\
\hline & & 4 & 208 & 46,0 & 0,84 & 13,8431 & 2,1285 & 1,6091 & 3,8358 & 0,1616 & 3,191 & 0,83 & 993 & 43 & 974 & 24 & 965 & 29 \\
\hline & & 6 & 238 & 54,5 & 0,99 & 13,9045 & 1,4154 & 1,5787 & 2,8967 & 0,1592 & 2,5274 & 0,87 & 984 & 29 & 962 & 18 & 952 & 22 \\
\hline & & 7 & 233 & 53,9 & 0,96 & 13,7555 & 2,2831 & 1,6106 & 3,8519 & 0,1607 & 3,1023 & 0,81 & 1006 & 46 & 974 & 24 & 961 & 28 \\
\hline & & 8 & 361 & 78,5 & 0,72 & 14,0987 & 2,1816 & 1,5757 & 4,5469 & 0,1611 & 3,9894 & 0,88 & 955 & 45 & 961 & 28 & 963 & 36 \\
\hline & & 9 & 145 & 31,6 & 0,72 & 13,6996 & 2,501 & 1,6084 & 5,0415 & 0,1598 & 4,3774 & 0,87 & 1014 & 51 & 973 & 32 & 956 & 39 \\
\hline & & 11 & 127 & 29,6 & 0,89 & 13,8189 & 3,0497 & 1,6502 & 4,5651 & 0,1654 & 3,3971 & 0,74 & 996 & 62 & 990 & 29 & 987 & 31 \\
\hline & & 14 & 179 & 37,8 & 0,71 & 13,9576 & 2,2324 & 1,5789 & 3,8929 & 0,1598 & 3,1892 & 0,82 & 976 & 46 & 962 & 24 & 956 & 28 \\
\hline & & 15 & 159 & 35,9 & 0,96 & 13,8288 & 2,9589 & 1,5854 & 4,1728 & 0,159 & 2,9423 & 0,71 & 995 & 60 & 965 & 26 & 951 & 26 \\
\hline & & 17 & 179 & 39,0 & 0,79 & 13,7986 & 1,9503 & 1,6059 & 3,7327 & 0,1607 & 3,1826 & 0,85 & 999 & 40 & 973 & 23 & 961 & 28 \\
\hline & & 18 & 243 & 60,6 & 1,40 & 13,9929 & 2,3186 & 1,5449 & 4,2935 & 0,1568 & 3,6137 & 0,84 & 971 & 47 & 948 & 26 & 939 & 32 \\
\hline & & 19 & 241 & 48,9 & 0,76 & 13,8187 & 2,2561 & 1,5659 & 4,2097 & 0,1569 & 3,5541 & 0,84 & 996 & 46 & 957 & 26 & 940 & 31 \\
\hline & & 20 & 353 & 72,8 & 0,77 & 13,5901 & 1,9303 & 1,59 & 4,1961 & 0,1567 & 3,7257 & 0,89 & 1030 & 39 & 966 & 26 & 939 & 33 \\
\hline
\end{tabular}




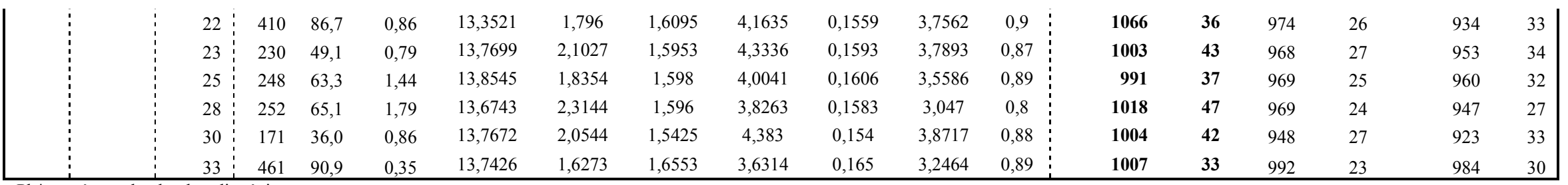

$\mathrm{Pb}^{*}$ représente le plomb radiogénique. 
Tableau 5 : Ratios des divers isotopes uranium et plomb avec les âges isotopiques calculés pour les grains de zircon de l'échantillon 12SJ14 par CA-ID-TIMS (en gras = dates utilisées pour calculer les âges concordia des phases de déformation; modifié de Jannin et al., accepté pour publication).

\begin{tabular}{|c|c|c|c|c|c|c|c|c|c|c|c|c|c|c|c|c|c|c|c|}
\hline $\begin{array}{l}\text { Groupe } \\
\text { CL }\end{array}$ & $\begin{array}{l}\mathrm{N}^{\circ} \\
\text { d'An } \\
\text { alyse }\end{array}$ & $\begin{array}{c}\text { Concentr } \\
\text { ion } U \\
\text { (ppm) }\end{array}$ & $\mathrm{Th} / \mathrm{U}$ & $\begin{array}{c}\text { Concen } \\
\text { tration } \\
\mathrm{Pb} \\
\text { totale } \\
(\mathrm{ppm})\end{array}$ & $\begin{array}{l}\mathrm{Pb}^{* /} \\
\mathrm{Pb}_{\mathrm{c}}\end{array}$ & $\begin{array}{l}\mathrm{Pb}_{\mathrm{c}} \\
(\mathrm{pg})\end{array}$ & ${ }^{207} \mathrm{~Pb} /{ }^{206} \mathrm{~Pb}$ & $\begin{array}{l} \pm \\
2 \sigma \\
(\%)\end{array}$ & $\begin{array}{l}{ }^{207} \mathrm{~Pb} /{ }^{235} \\
\mathrm{U}\end{array}$ & $\begin{array}{l} \pm 2 \sigma \\
(\%)\end{array}$ & ${ }^{206} \mathrm{~Pb} /{ }^{238} \mathrm{U}$ & $\begin{array}{l} \pm 2 \sigma \\
(\%)\end{array}$ & rho & $\begin{array}{l}\text { Age }(\mathrm{Ma}) \\
{ }^{207} \mathrm{~Pb} /{ }^{206} \mathrm{~Pb}\end{array}$ & $\begin{array}{l} \pm 2 \sigma \\
(\mathrm{Ma})\end{array}$ & $\begin{array}{l}\text { Age (Ma) } \\
{ }^{207} \mathrm{~Pb} /{ }^{235} \mathrm{U}\end{array}$ & $\begin{array}{l} \pm 2 \sigma \\
(\mathrm{Ma})\end{array}$ & $\begin{array}{l}\text { Age } \\
(\mathrm{Ma}) \\
{ }^{206} \mathrm{~Pb} /{ }^{23} \\
{ }^{8} \mathrm{U}\end{array}$ & $\begin{array}{l} \pm 2 \sigma \\
(\mathrm{Ma})\end{array}$ \\
\hline \multicolumn{20}{|c|}{ Echantillon 12SJ14 } \\
\hline groupe & 6 & 523 & 0,02 & 86,2 & 13,2 & 6,08 & 0,072074233 & 0,37 & 1,6425 & 0,61 & 0,165283832 & 0,46 & 0,799 & 985,3 & 7,5 & 986,7 & 3,9 & 986,1 & 4,2 \\
\hline 2 & 3 & 117 & n.m. & 19,4 & 7,6 & 2,26 & 0,072000106 & 0,86 & 1,6064 & $0,97$. & 0,161819824 & $0,37$. & 0,485 & 983,2 & 17,4 & 972,7 & 6,1 & 966,9 & 3,4 \\
\hline groupe & 1 & 128 & 0,02 & 21,0 & 20,0 & 2,00 & 0,072639316 & 0,27 & 1,679 & 0,48 & 0,167641411 & 0,37 & 0,834 & 1001,1 & 5,5 & 1000,6 & 3,1 & 999,1 & 3,4 \\
\hline 1 & 5 & 461 & 0,02 & 75,9 & 18,5 & 3,88 & 0,072631153 & 0,18 & 1,6877 & 0,41 & 0,168524486 & 0,33 & 0,91 & 1000,9 & $3,6$. & 1003,9 & 2,6 & 1004,0 . & 3,1 \\
\hline 1 ou 2 & 2 & 142 & 0,03 & 26,4 & 5,4 & 4,12 & 0,073298842 & 0,45 & 1,7011 & 0,61 & 0,168317699 & 0,36 & 0,671 & 1019,4 & 9,2 & 1008,9 & 3,9 & 1002,8 & 3,3 \\
\hline +hérité & 4 & 80 & 0,01 & 14,3 & 6,5 & 1,9 & 0,072931402 & 0,48 & 1,6753 & 0,47 & 0,166604449 & 0,26 & 0,229 & 1009,5 & 9,7 & 999,2 & 3,0 & 993,4 & 2,4 \\
\hline
\end{tabular}

$\mathrm{Pb}^{*}$ représente le plomb radiogénique.

$\mathrm{Pb}_{\mathrm{c}}$ représente le plomb commun. 


\section{Liste des illustrations :}

Figure 1: A) Répartition du métamorphisme ottawan et rigolet en fonction des conditions de pression qui définissent les subdivisions de la Province de Grenville; la répartition des déformation anté-grenvilliennes dans la Ceinture parautochtone a été ajoutée à la carte (modifiée de Rivers, 2008 et de Rivers et al., 2012). B) Schéma structural de la région d'étude, au SO du réservoir Manicouagan (interprétation de la carte géologique de Moukhsil et al., 2013; les dates correspondent à des âges de cristallisation ou de dépôt sur zircon détritique) où figurent les plis polyphasés, les intensités des déformations et les secteurs tectoniques (modifié de Jannin et al., 2013, 2014, accepté pour publication).

Figure 2 : Stéréogrammes des pôles des foliations S1, S2, des linéations L1 à L3 et des axes de plis A1, A2; les linéations L2 et L3 ont pu être déterminées à l'affleurement lorsque la L2 était déformée par des cisaillements locaux (modifié de Jannin et al., accepté pour publication).

Figure 3 : Carte géologique d'une portion du secteur $\mathrm{B}$ réalisée à partir des observations de terrain et de l'interprétation des cartes du gradient magnétique et du tilt.

Figure 4 : Schéma illustrant les différentes phases de déformation cartographiées dans la région d'étude.

Figure 5 : Caractéristiques des déformations D1 à D3 (modifié de Jannin et al., 2013, 2014, accepté pour publication). A) Affleurement 12SJ14, paragneiss du Groupe de Gagnon. A1) Leucosomes mis en place dans la schistosité de plan axial S2 peu pénétrative, associée à un cisaillement apparent senestre et recoupant la foliation S1. A2) Interconnexion des leucosomes d'épaisseur variable, bordés par des mélanosomes. B) Foliation S1 plissée par la déformation D2 dans les gneiss archéens. C) Porphyroclaste de type $\delta$ composé de clinopyroxène et d'amphibole et boudin de type shear-band, indiquant un cisaillement $\mathrm{C} 2$ senestre, dans les gneiss archéens. D) 
Affleurement 13SJ93, gneiss archéens. D1) Transposition des axes de plis, parallèles à la linéation L2. D2) Porphyroclaste de type $\sigma$ indiquant un cisaillement C2 inverse-dextre. E) Affleurement 12SJ27, gneiss archéens. E1) Veine au cœur d'un flanking shear-band indiquant un cisaillement C2 inverse; la veine était partiellement fondue pendant la rotation, or lorsqu'un élément, ayant une viscosité beaucoup plus faible que l'encaissant qu'il recoupe, subit une rotation lors d'un cisaillement, tel que ce dyke granitique, aux marges de cet élément la foliation est entraînée en mouvement normal (Passchier, 2001). E2) Foliation S2 très pénétrative. E3) Cisaillement tardif senestre-normal. F) Affleurement 13SJ92, gneiss archéens. F1) Mise en place de leucosomes dans la schistosité de plan axial S2 et dans la foliation S1. F2) Stéréogramme des structures de l'affleurement. G) Affleurement 13SJ49, paragneiss du Groupe de Gagnon. G1) Déformation D3 pénétrative, transposant la foliation D2 dans le secteur E. G2) Leucosome porphyroclastique de type $\delta$ recoupant la foliation et marqué par un enroulement de queue indiquant un cisaillement C3 dextre-normal.

Figure 6 : Photographies macroscopiques (A) et microscopiques (B à G) de l'échantillon 13SJ182 (section XY; modifié de Jannin et al., accepté pour publication). A) Présence de kyanite dans les leucosomes et dans la matrice. B) Remplacement d'une kyanite et du quartz par un grenat (en LPA). C) Couronne de phyllosilicates dont de la muscovite autour de kyanite en contact avec la biotite; remplacement de la biotite par la muscovite et forme globulaire à polygonale du quartz (en LPA). D) Deux générations de biotite chloritisées respectivement parallèle et perpendiculaire à la foliation (en LPNA). E) Forme globulaire à polygonale du quartz recristallisé (en LPNA). F) Remplacement de biotite, kyanite et quartz par un grenat (en LPA). G) Remplacement partiel de la biotite par la muscovite (en LPA).

Figure 7 : A) Photographies macroscopique (A1) et microscopiques (A2 et A3) de l'échantillon 13SJ100B (section XZ). A1) Porphyroclaste de type $\delta$ avec ombre de pression à sillimanite 
indiquant un cisaillement C2 senestre-inverse. A2) Muscovite rétrograde en contact avec la biotite entièrement chloritisée contenant de la sillimanite (en LPA). A3) Biotite contenant des fabriques $\mathrm{C} / \mathrm{S}$ ondulées en présence des plans $\mathrm{C}^{\prime}$, indiquant un cisaillement $\mathrm{C} 2$ senestre-inverse (en LPA; Berthé et al., 1979; Simpson et Schmid, 1983); sillimanite et rubans de quartz contenus dans la biotite chloritisée. B) Photographies microscopiques de l'échantillon 13SJ102A (section XZ). B1) Intercroissances lobées de quartz dans le grenat, indicateur de fusion partielle (en LPNA; Waters, 2001). B2) Forme idiomorphe du grenat (en LPNA). B3) Biotite faiblement chloritisée contenant de la sillimanite dans ses plans de clivage (en LPA).

Figure 8 : Photographies microscopiques non orientées en LPA de l'échantillon 12SJ27F (modifié de Jannin et al., accepté pour publication). A) Clinopyroxène corrodé et hornblende. B) Remplacement partiel de hornblende par la chlorite. C) Hornblende à inclusion de clinopyroxène.

Figure 9 : Géochronologie U/Pb et analyses ÉTR sur zircon de l'échantillon 12SJ14 (modifié de Jannin et al., 2013, 2014, accepté pour publication). A) Leucosome syn-D2 échantillonné dans un paragneiss. B) Images en cathodoluminescence des grains de zircon présentant deux surcroissances (groupes 1 et 2) et des cœurs hérités. C) Diagramme géochimique discriminant les groupes 1 et 2. D) Concentrations en ÉTR normalisées aux chondrites (Sun et McDonough, 1989). D1) Patron des noyaux hérités. D2) et D3) Patrons identiques d'une majorité des analyses des groupes 1 et 2. D4) Patrons de huit analyses différentes difficilement interprétables. E) Âges ${ }^{207} \mathrm{~Pb} /{ }^{206} \mathrm{~Pb}$ de $995 \pm 21 \mathrm{Ma}$ pour le groupe 2 et de $1008 \pm 18$ Ma pour le groupe 1 , obtenus par LA-ICP-MS. F) Diagramme concordia donnant deux âges de cristallisation de $986 \pm 4$ Ma pour le groupe 2 et de $1002 \pm 2$ Ma pour le groupe 1, obtenus par ID-TIMS.

Figure 10 : Géochronologie U/Pb et analyses ÉTR sur zircon de l'échantillon 12SJ27E (modifié de Jannin et al., 2013, 2014, accepté pour publication). A1) et A2) Dyke de gabbro composite recoupant la S2 du gneiss archéen, provenant du Complexe de Ulamen, et se mettant parfois en 
place dans la S2; d'après les évidences de mélange de magma (fragments arrondis de gabbro et de pegmatite continu respectivement dans de la pegmatite et du gabbro), la cristallisation de ce dyke de gabbro est synchrone à celle de la pegmatite. A3) Grains de hornblende marquant la linéation L2 dans ce dyke de gabbro. B) Pegmatite syn-D2 échantillonnée. C) Images en cathodoluminescence des grains de zircon, présentant deux surcroissances (groupes 1 et 2). D) Concentrations en ÉTR normalisées aux chondrites (Sun et McDonough, 1989). D1) et D2) Patrons identiques d'une majorité des analyses des groupes 1 et 2. D3) Patrons de cinq analyses différentes difficilement interprétables. E) Âges ${ }^{207} \mathrm{~Pb} /{ }^{206} \mathrm{~Pb}$ de $961 \pm 22 \mathrm{Ma}$ pour le groupe 2 et de $1001 \pm 16$ Ma pour le groupe 1 , obtenus par LA-ICP-MS.

Figure 11 : A) Schéma illustrant les différentes phases de déformation et de métamorphisme avec leur âge dans la Ceinture parautochtone. B) Chemins P-T schématiques des secteurs B et D, inspirés de Jordan et al. (2006) illustrant la chronologie relative entre le métamorphisme, la déformation et la cristallisation des grains de zircon de l'échantillon 12SJ14. Puisque l'intervalle de température dans lequel la réaction $\mathrm{R} 1$ est continue est faible, cette réaction forme globalement une droite dans le champ P-T, alors que la réaction R2 est continue sur un intervalle de température plus grand et se déroule donc dans la zone illustrée en teinte de gris. C) Tableau illustrant l'évolution tectono-métamorphique de la Ceinture parautochtone (modifié de Jannin et al., accepté pour publication).

\section{Liste des illustrations de l'appendice 1 :}

Figure A1 : Illustration des valeurs de l'intensité de la déformation (voir le texte de l'appendice 1 pour les caractéristiques de chaque intensité; la valeur 5 correspond aux gneiss droits définis par Hanmer, 1988). 


\section{Appendice 1}

L'intensité de la déformation a été évaluée de façon qualitative en observant les faces perpendiculaires à la foliation, en estimant une moyenne de l'intensité sur l'affleurement. Cette évaluation tient compte de la rhéologie des lithologies, puisque pour un affleurement de gabbro ou de paragneiss et une intensité réelle identique, l'intensité de la déformation apparente sera différente en raison de la différence en homogénéité et en compétence des lithologies. Tous les affleurements cartographiés sont déformés, l'intensité la plus faible a donc une valeur entre 1 et 2. L'évaluation de l'intensité de la déformation se base sur plusieurs critères : la foliation, la linéation minérale ou d'étirement, la forme des plis, ouverts, serrés ou isoclinaux, la taille et la forme des grains et des objets tel que les boudins, les leucosomes, etc. Ces critères correspondent donc pour chaque intensité à (Fig. A1) :

- 1-2 : une foliation très discontinue, ondulée dont le plan est difficilement identifiable; une linéation très peu identifiable; des plis ondulants à ouverts; une grande majorité de grains non déformés, non orientés, non étirés et pluri-millimétriques; des leucosomes parallèles à la foliation ayant des limites et une continuité mal définies.

- 2 : une foliation discontinue, ondulée dont le plan est identifiable; une linéation identifiable mais peu marquée; des plis ouverts à serrés; une majorité de grains peu déformés, peu orientés, très peu étirés et pluri-millimétriques; des leucosomes parallèles à la foliation et discontinus avec des limites identifiables à mal définies.

- 3 : une foliation continue, rectiligne à plissée; une linéation modérément marquée; des plis serrés à isoclinaux; une petite majorité de grains déformés, orientés, peu étirés et millimétriques à pluri-millimétriques; des leucosomes parallèles à la foliation, 
majoritairement continus avec des limites généralement bien définies; des boudins existants mais peu étirés.

- 4 : une foliation continue et rectiligne; une linéation bien marquée; des plis démembrés et boudinés dont la charnière sont encore identifiable; une majorité de grains déformés, orientés, étirés et millimétriques; des leucosomes parallèles à la foliation, boudinés avec des limites très bien définies et une forme ellipsoïde; des boudins étirés dont la forme ellipsoïde est toujours reconnaissable.

- 5 : une foliation continue et rectiligne; une linéation très bien marquée; des plis si démembrés et boudinés qu'ils ne sont plus identifiables; une grande majorité de grains très déformés, orientés, très étirés et infra-millimétriques à millimétriques; des leucosomes parallèles à la foliation, boudinés avec des limites très bien définies et une forme rectangulaire et très allongée; des boudins étirés dont la forme ellipsoïde n'est plus reconnaissable. Ce type de roche correspond à un gneiss droit défini par Hanmer (1988). 


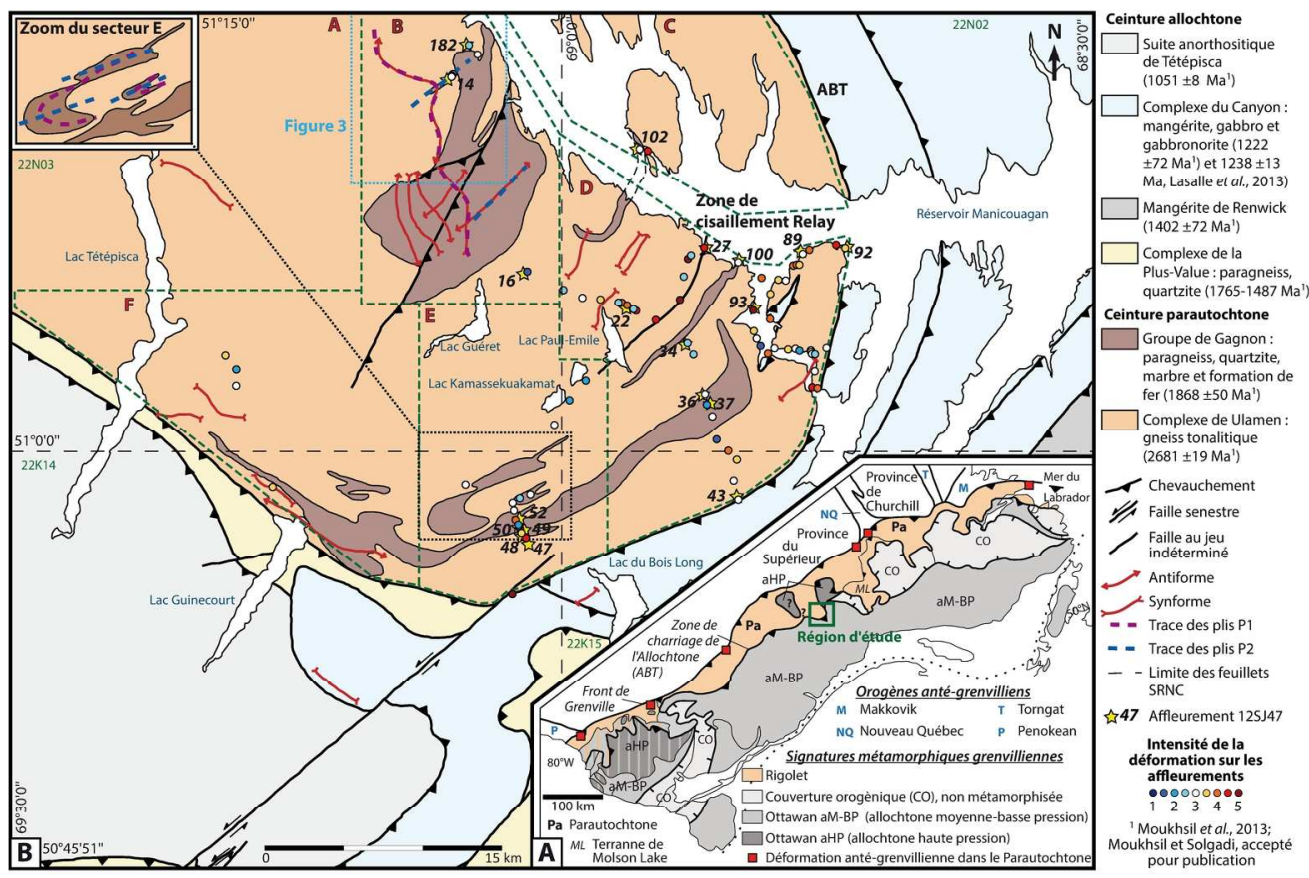

Figure 1: A) Répartition du métamorphisme ottawan et rigolet en fonction des conditions de pression qui définissent les subdivisions de la Province de Grenville; la répartition des déformation anté-grenvilliennes dans la Ceinture parautochtone a été ajoutée à la carte (modifiée de Rivers, 2008 et de Rivers et al., 2012).

B) Schéma structural de la région d'étude, au SO du réservoir Manicouagan (interprétation de la carte géologique de Moukhsil et al., 2013; les dates correspondent à des âges de cristallisation ou de dépôt sur zircon détritique) où figurent les plis polyphasés, les intensités des déformations et les secteurs tectoniques (modifié de Jannin et al., 2013, 2014, accepté pour publication).

$181 \times 139 \mathrm{~mm}(300 \times 300 \mathrm{DPI})$ 


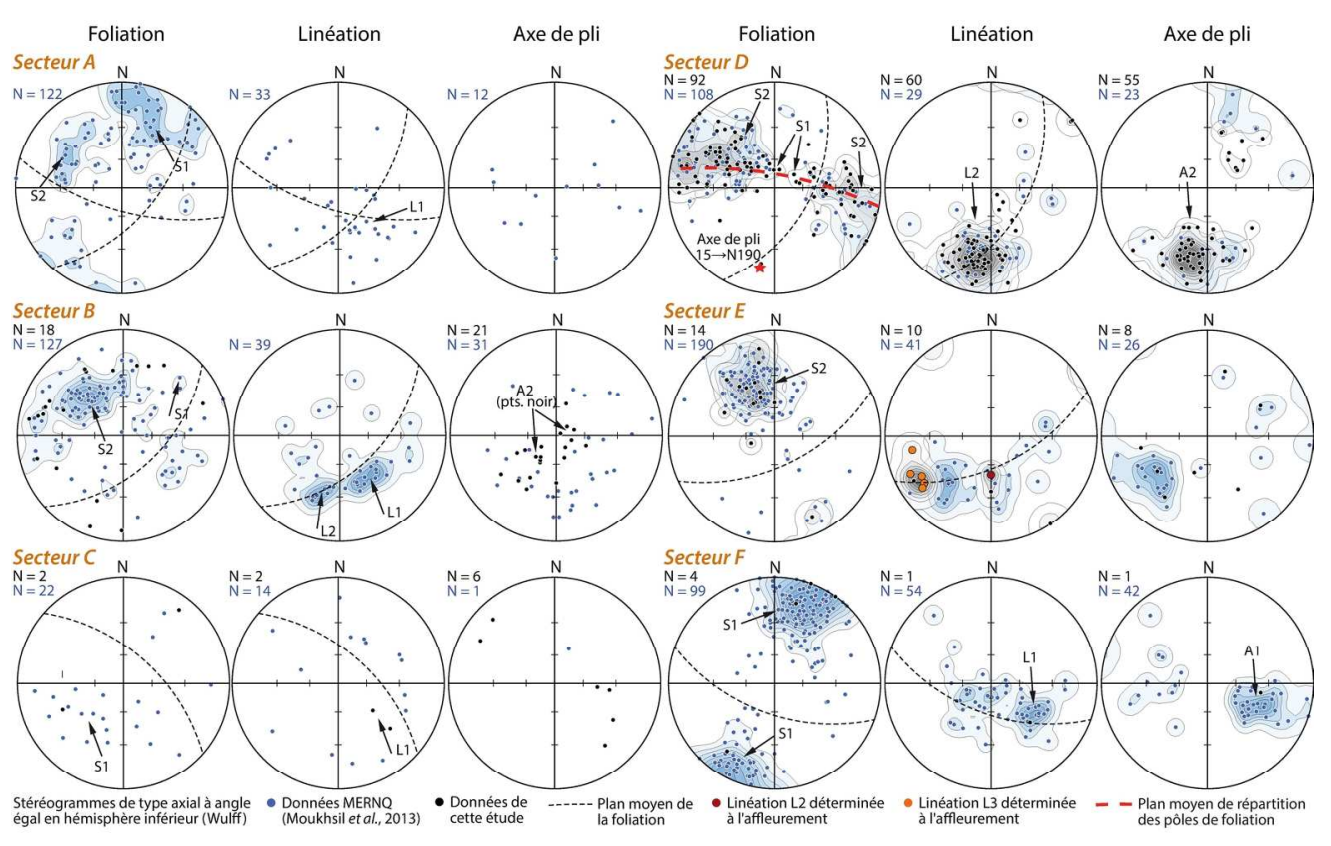

Figure 2 : Stéréogrammes des pôles des foliations $S 1, S 2$, des linéations $L 1$ à $L 3$ et des axes de plis $A 1, A 2$; les linéations $L 2$ et $L 3$ ont pu être déterminées à l'affleurement lorsque la $L 2$ était déformée par des cisaillements locaux (modifié de Jannin et al., accepté pour publication).

$181 \times 139 \mathrm{~mm}(300 \times 300 \mathrm{DPI})$ 


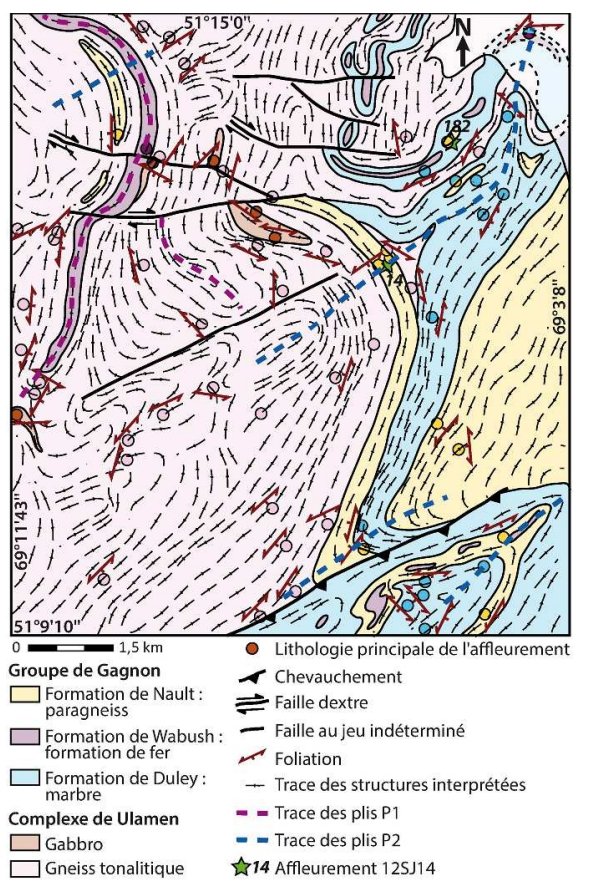

Figure 3 : Carte géologique d'une portion du secteur $B$ réalisée à partir des observations de terrain et de l'interprétation des cartes du gradient magnétique et du tilt.

$236 \times 653 \mathrm{~mm}(300 \times 300 \mathrm{DPI})$ 


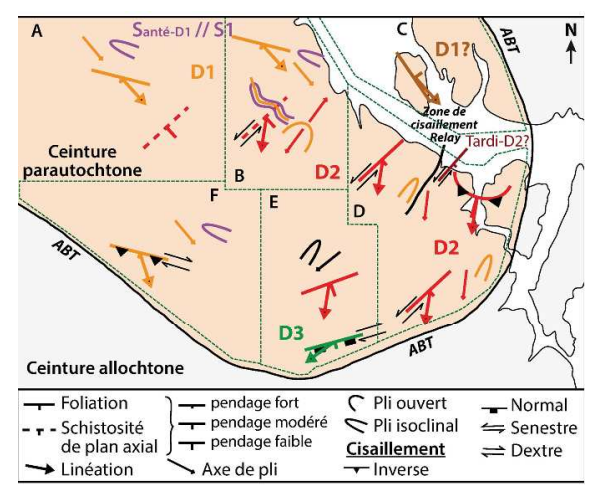

Figure 4 : Schéma illustrant les différentes phases de déformation cartographiées dans la région d'étude. $236 \times 653 \mathrm{~mm}(300 \times 300 \mathrm{DPI})$ 

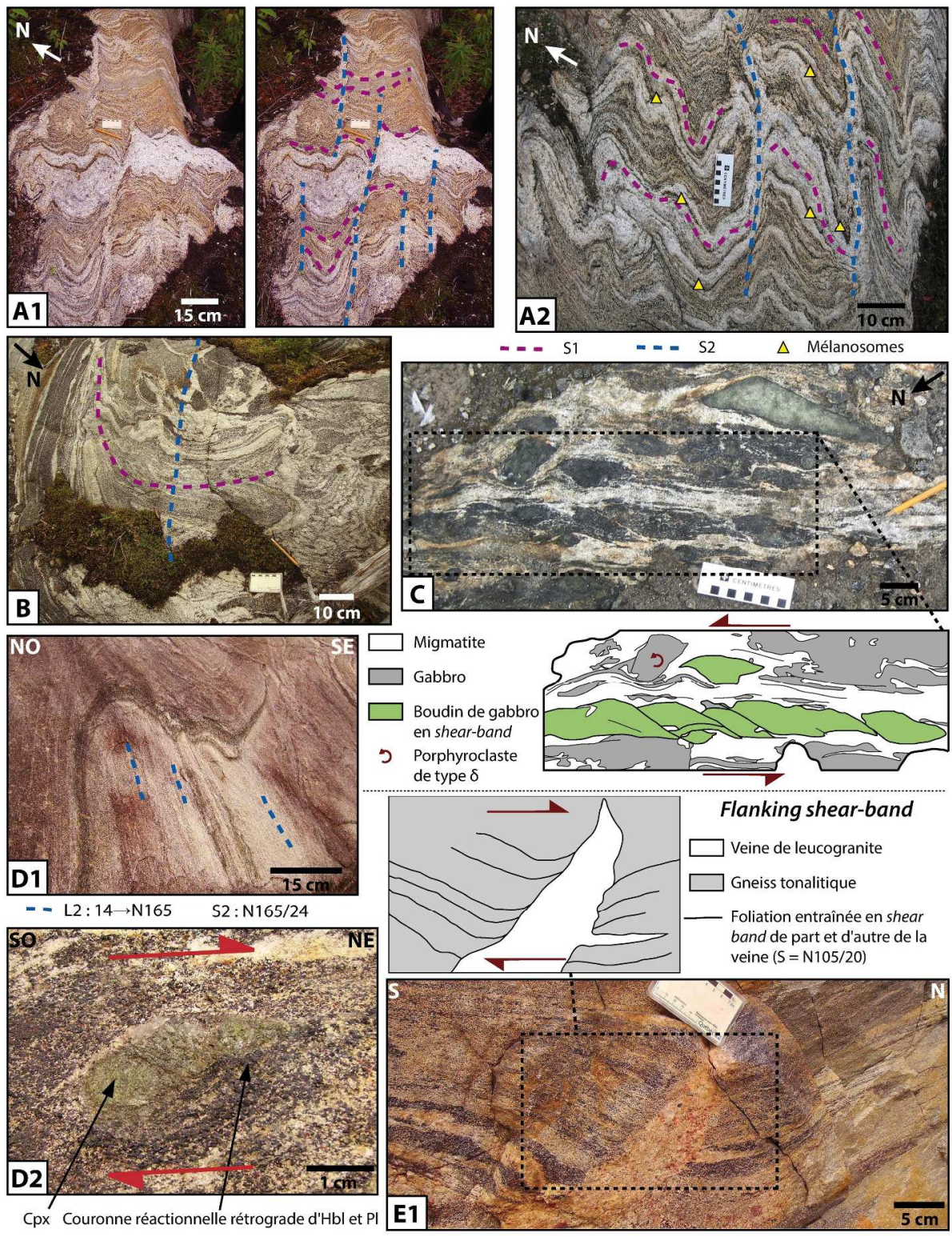

Figure 5 : Caractéristiques des déformations D1 à D3 (modifié de Jannin et al., 2013, 2014, accepté pour publication). A) Affleurement 12SJ14, paragneiss du Groupe de Gagnon. A1) Leucosomes mis en place dans la schistosité de plan axial S2 peu pénétrative, associée à un cisaillement apparent senestre et recoupant la foliation S1. A2) Interconnexion des leucosomes d'épaisseur variable, bordés par des mélanosomes. B) Foliation S1 plissée par la déformation D2 dans les gneiss archéens. C) Porphyroclaste de type $\delta$ composé de clinopyroxène et d'amphibole et boudin de type shear-band, indiquant un cisaillement C2 senestre, dans les gneiss archéens. D) Affleurement 13S193, gneiss archéens. D1) Transposition des axes de plis, parallèles à la linéation L2. D2) Porphyroclaste de type $\sigma$ indiquant un cisaillement C2 inverse-dextre. E) Affleurement 12SJ27, gneiss archéens. E1) Veine au cœur d'un flanking shear-band indiquant un cisaillement C2 inverse; la veine était partiellement fondue pendant la rotation, or lorsqu'un élément, ayant une viscosité beaucoup

plus faible que l'encaissant qu'il recoupe, subit une rotation lors d'un cisaillement, tel que ce dyke granitique, aux marges de cet élément la foliation est entraînée en mouvement normal (Passchier, 2001). E2) Foliation S2 très pénétrative. E3) Cisaillement tardif senestre-normal. F) Affleurement 13SJ92, gneiss 
archéens. F1) Mise en place de leucosomes dans la schistosité de plan axial S2 et dans la foliation S1. F2) Stéréogramme des structures de l'affleurement. G) Affleurement 13SJ49, paragneiss du Groupe de Gagnon. G1) Déformation D3 pénétrative, transposant la foliation D2 dans le secteur E. G2) Leucosome porphyroclastique de type $\delta$ recoupant la foliation et marqué par un enroulement de queue indiquant un cisaillement C3 dextre-normal.

$236 \times 308 \mathrm{~mm}(300 \times 300 \mathrm{DPI})$ 


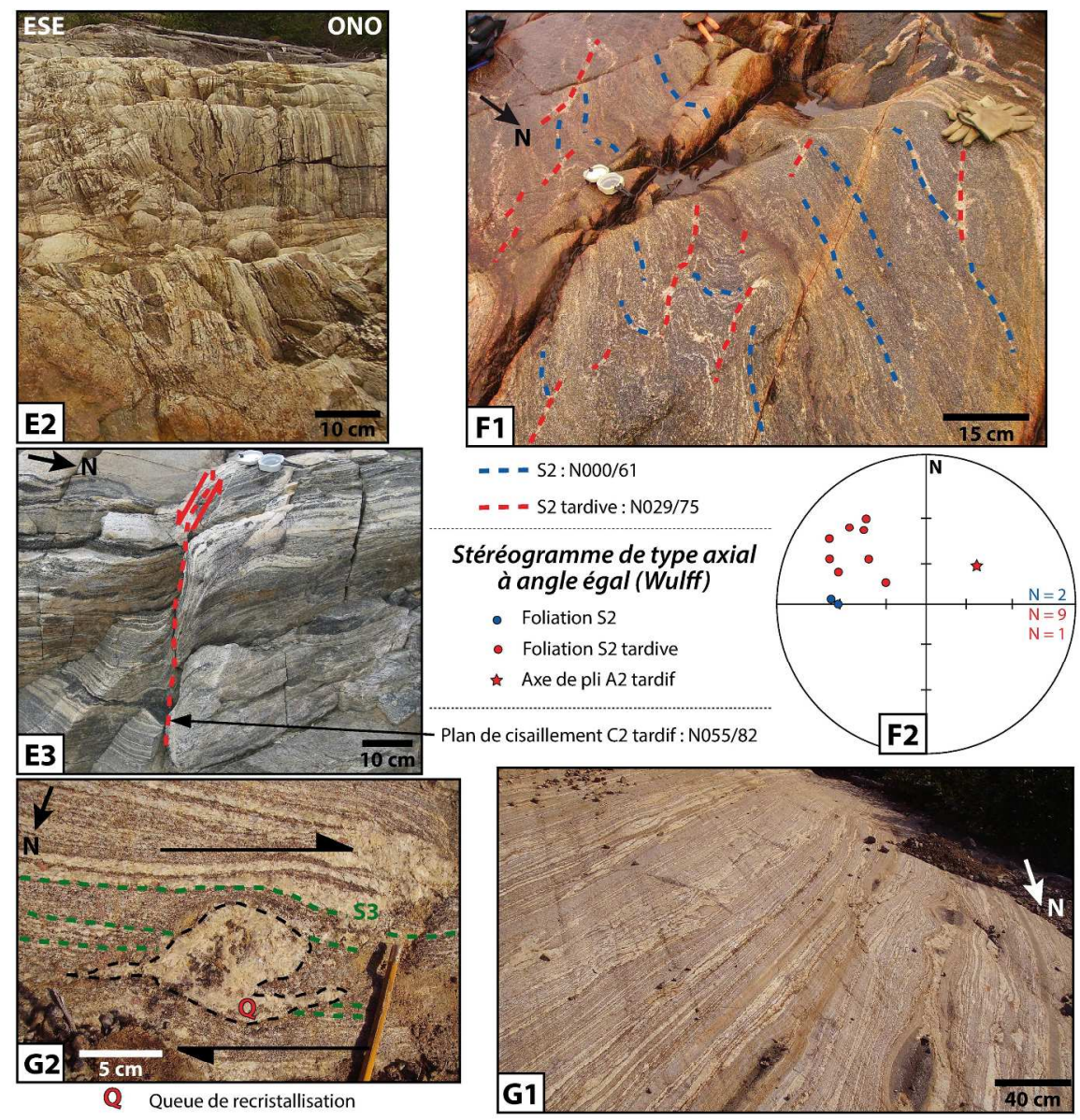

Figure 5 : suite

$236 \times 308 \mathrm{~mm}(300 \times 300$ DPI $)$ 

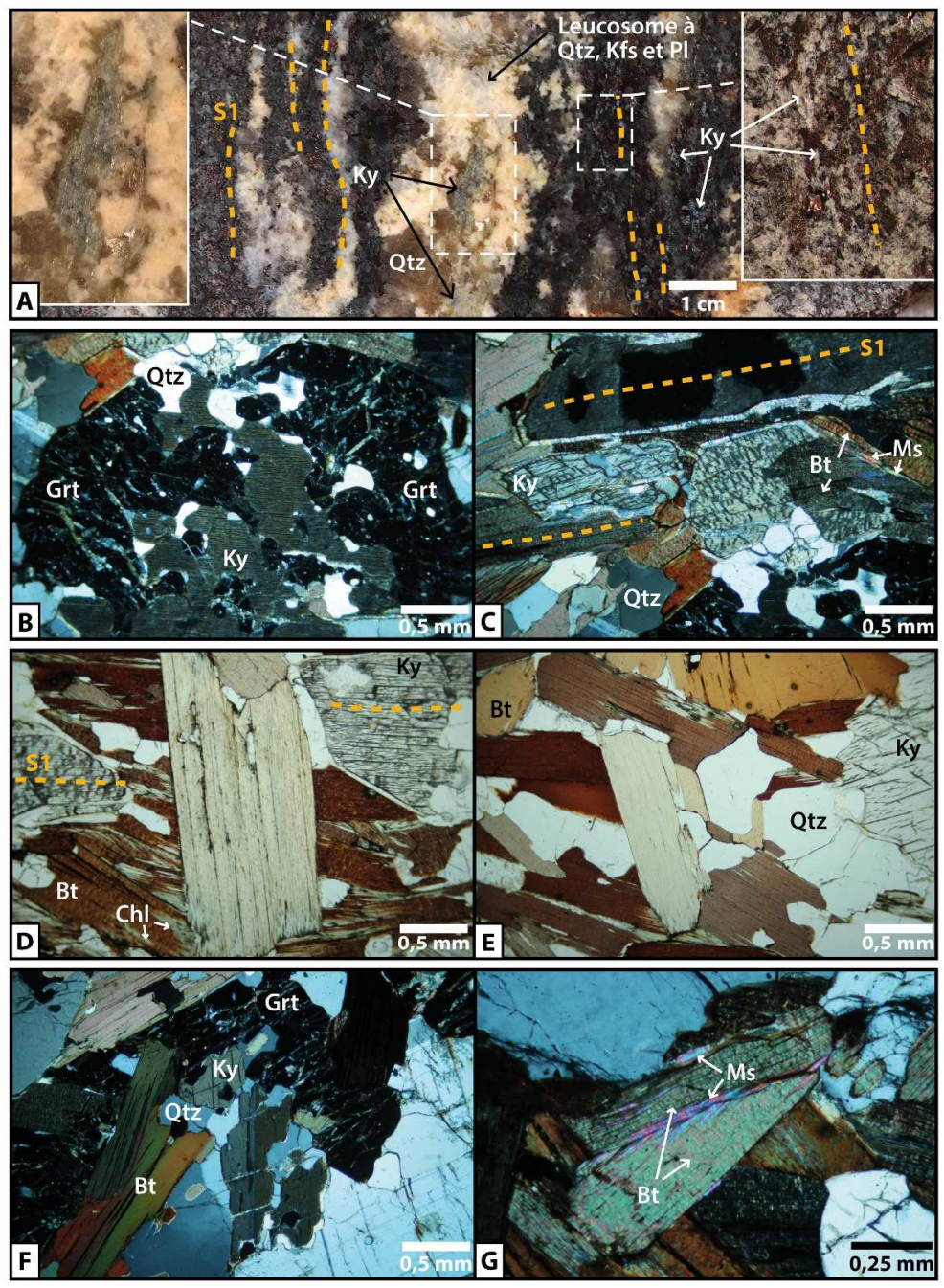

Figure 6 : Photographies macroscopiques (A) et microscopiques (B à G) de l'échantillon 135 S182 (section $X Y$; modifié de Jannin et al., accepté pour publication). A) Présence de kyanite dans les leucosomes et dans la matrice. B) Remplacement d'une kyanite et du quartz par un grenat (en LPA). C) Couronne de phyllosilicates dont de la muscovite autour de kyanite en contact avec la biotite; remplacement de la biotite par la muscovite et forme globulaire à polygonale du quartz (en LPA). D) Deux générations de biotite chloritisées respectivement parallèle et perpendiculaire à la foliation (en LPNA). E) Forme globulaire à polygonale du quartz recristallisé (en LPNA). F) Remplacement de biotite, kyanite et quartz par un grenat (en LPA). G) Remplacement partiel de la biotite par la muscovite (en LPA).

$$
236 \times 308 \mathrm{~mm}(300 \times 300 \text { DPI) }
$$


d'Adobe Illustrator avec l'option Créer un fichier compatible PDF activée. Cette option se trouve dans la boîte de dialogue d'options du format natif Illustrator, qui apparaît lors de l'enregistrement d'un fichier Adobe Illustrator à l'aide de la commande Enregistrer sous.

II s'agit d'un fichier Adobe ${ }^{\oplus}$ |llustrator ${ }^{\oplus}$ qui a été enregistré sans contenu PDF.

Pour importer ou ouvrir ce fichier dans d'autres applications, il doit être à nouveau enregistré à partir d'Adobe Illustrator avec l'option Créer un fichier compatible PDF activée. Cette option se trouve dans la boite de dialogue d'options du format natif Illustrator, qui apparaît lors de l'enregistrement d'un fichier Adobe Illustrator à l'aide de la commande Enregistrer sous.

II s'agit d'un fichier Adobe ${ }^{\circledast}$ |llustrator ${ }^{\circledast}$ qui a été enregistré sans contenu PDF.

Pour importer ou ouvrir ce fichier dans d'autres applications, il doit être à nouveau enregistré à partir d'Adobe Illustrator avec l'option Créer un fichier compatible PDF activée. Cette option se trouve dans la boîte de dialogue d'options du format natif Illustrator, qui apparaît lors de l'enregistrement d'un fichier Adobe Illustrator à l'aide de la commande Enregistrer sous.

II s'agit d'un fichier Adobe ${ }^{\circledast}$ Illustrator ${ }^{\circledast}$ qui a été enregistré sans contenu PDF.

Pour importer ou ouvrir ce fichier dans d'autres applications, il doit être à nouveau enregistré à partir d'Adobe Illustrator avec l'option Créer un fichier compatible PDF activée. Cette option se trouve dans la boite de dialogue d'options du format natif Illustrator, qui apparaît lors de l'enregistrement d'un fichier Adobe Illustrator à l'aide de la commande Enregistrer sous.

Figure $7: A$ ) Photographies macroscopique (A1) et microscopiques (A2 et $A 3$ ) de l'échantillon 13SJ100B (section $X Z$ ). A1) Porphyroclaste de type $\delta$ avec ombre de pression à sillimanite indiquant un cisaillement $C 2$ senestre-inverse. A2) Muscovite rétrograde en contact avec la biotite entièrement chloritisée contenant de la sillimanite (en LPA). A3) Biotite contenant des fabriques C/S ondulées en présence des plans $C^{\prime}$, indiquant un cisaillement C2 senestre-inverse (en LPA; Berthé et al., 1979; Simpson et Schmid, 1983); sillimanite et rubans de quartz contenus dans la biotite chloritisée. B) Photographies microscopiques de l'échantillon 13SJ102A (section XZ). B1) Intercroissances lobées de quartz dans le grenat, indicateur de fusion partielle (en LPNA; Waters, 2001). B2) Forme idiomorphe du grenat (en LPNA). B3) Biotite faiblement chloritisée contenant de la sillimanite dans ses plans de clivage (en LPA).

$236 \times 308 \mathrm{~mm}(300 \times 300 \mathrm{DPI})$ 


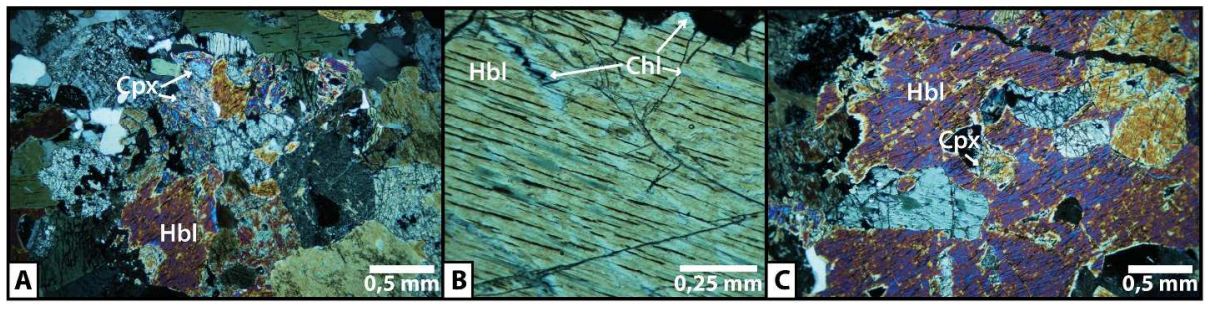

Figure 8 : Photographies microscopiques non orientées en LPA de l'échantillon 12SJ27F (modifié de Jannin et al., accepté pour publication). A) Clinopyroxène corrodé et hornblende. B) Remplacement partiel de hornblende par la chlorite. C) Hornblende à inclusion de clinopyroxène.

$$
236 \times 308 \mathrm{~mm} \text { ( } 300 \times 300 \text { DPI) }
$$



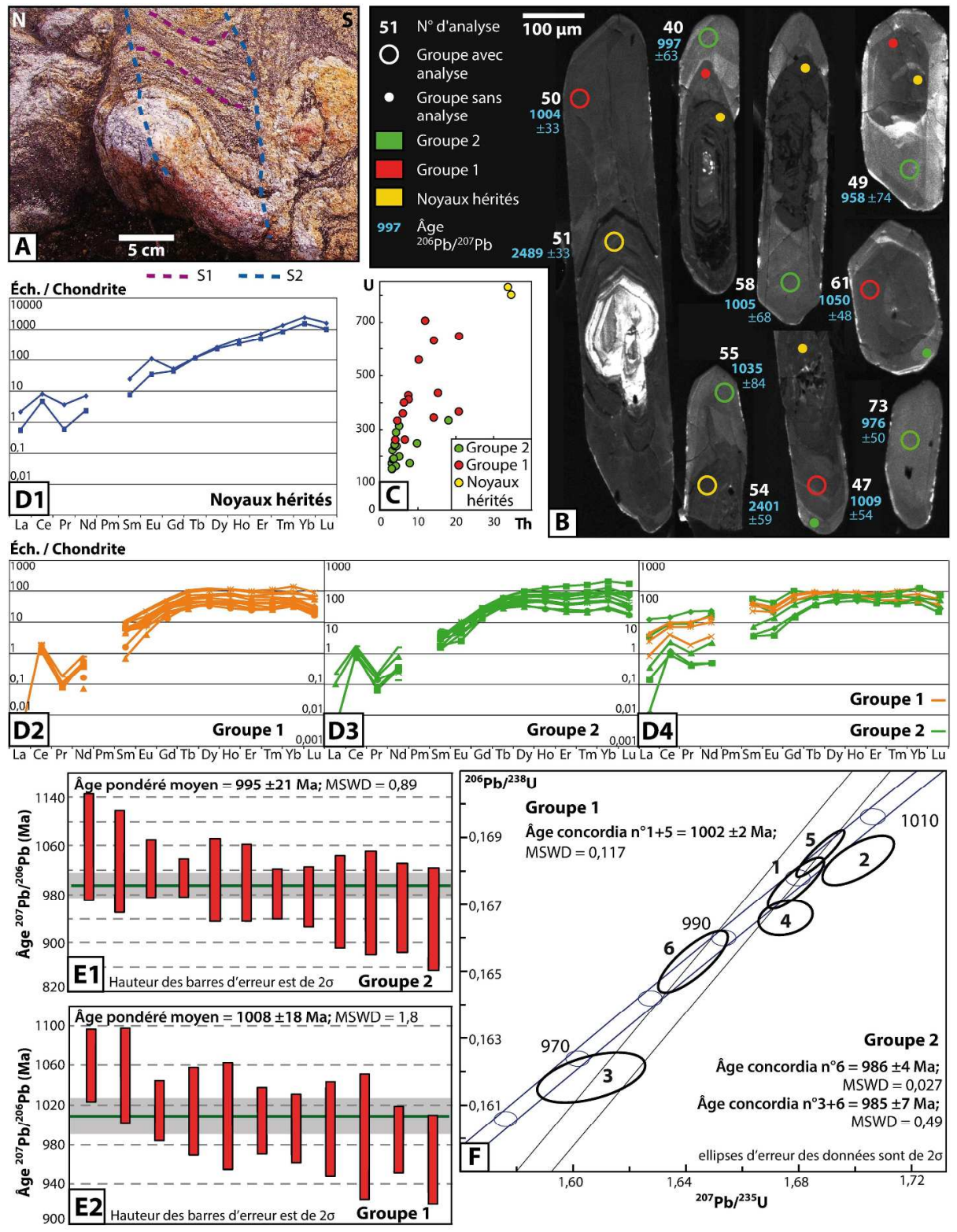

Figure 9 : Géochronologie U/Pb et analyses ÉTR sur zircon de l'échantillon 12SJ14 (modifié de Jannin et al., 2013, 2014, accepté pour publication). A) Leucosome syn-D2 échantillonné dans un paragneiss. B) Images en cathodoluminescence des grains de zircon présentant deux surcroissances (groupes 1 et 2) et des cœurs hérités. C) Diagramme géochimique discriminant les groupes 1 et 2. D) Concentrations en ÉTR normalisées aux chondrites (Sun et McDonough, 1989). D1) Patron des noyaux hérités. D2) et D3) Patrons identiques

d'une majorité des analyses des groupes 1 et 2. D4) Patrons de huit analyses différentes difficilement interprétables. E) Âges $207 \mathrm{~Pb} / 206 \mathrm{~Pb}$ de $995 \pm 21$ Ma pour le groupe 2 et de $1008 \pm 18$ Ma pour le groupe 1 , obtenus par LA-ICP-MS. F) Diagramme concordia donnant deux âges de cristallisation de $986 \pm 4$ Ma pour le groupe 2 et de $1002 \pm 2$ Ma pour le groupe 1, obtenus par CA-ID-TIMS.

\section{$236 \times 308 \mathrm{~mm}(300 \times 300 \mathrm{DPI})$}



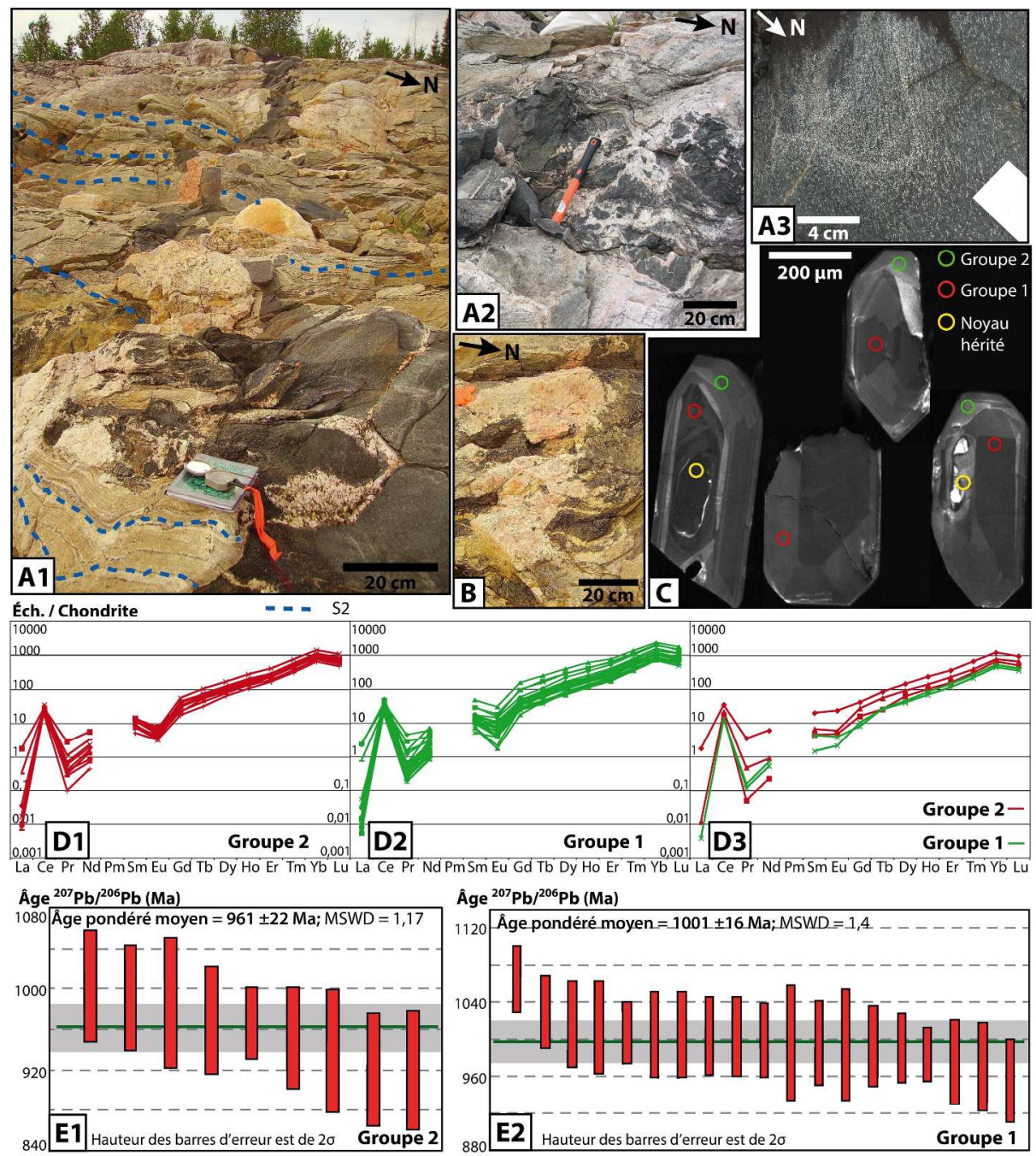

Figure 10 : Géochronologie U/Pb et analyses ÉTR sur zircon de l'échantillon 12SJ27E (modifié de Jannin et al., 2013, 2014, accepté pour publication). A1) et A2) Dyke de gabbro composite recoupant la S2 du gneiss archéen, provenant du Complexe de Ulamen, et se mettant parfois en place dans la S2; d'après les évidences de mélange de magma (fragments arrondis de gabbro et de pegmatite continu respectivement dans de la pegmatite et du gabbro), la cristallisation de ce dyke de gabbro est synchrone à celle de la pegmatite. A3) Grains de hornblende marquant la linéation L2 dans ce dyke de gabbro. B) Pegmatite syn-D2 échantillonnée. C) Images en cathodoluminescence des grains de zircon, présentant deux surcroissances (groupes 1 et 2). D) Concentrations en ÉTR normalisées aux chondrites (Sun et McDonough, 1989). D1) et D2) Patrons identiques d'une majorité des analyses des groupes 1 et 2. D3) Patrons de cinq analyses différentes difficilement interprétables. E) Âges 207Pb/206Pb de $961 \pm 22$ Ma pour le groupe 2 et de 1001 \pm 16 Ma pour le groupe 1 , obtenus par LA-ICP-MS. 


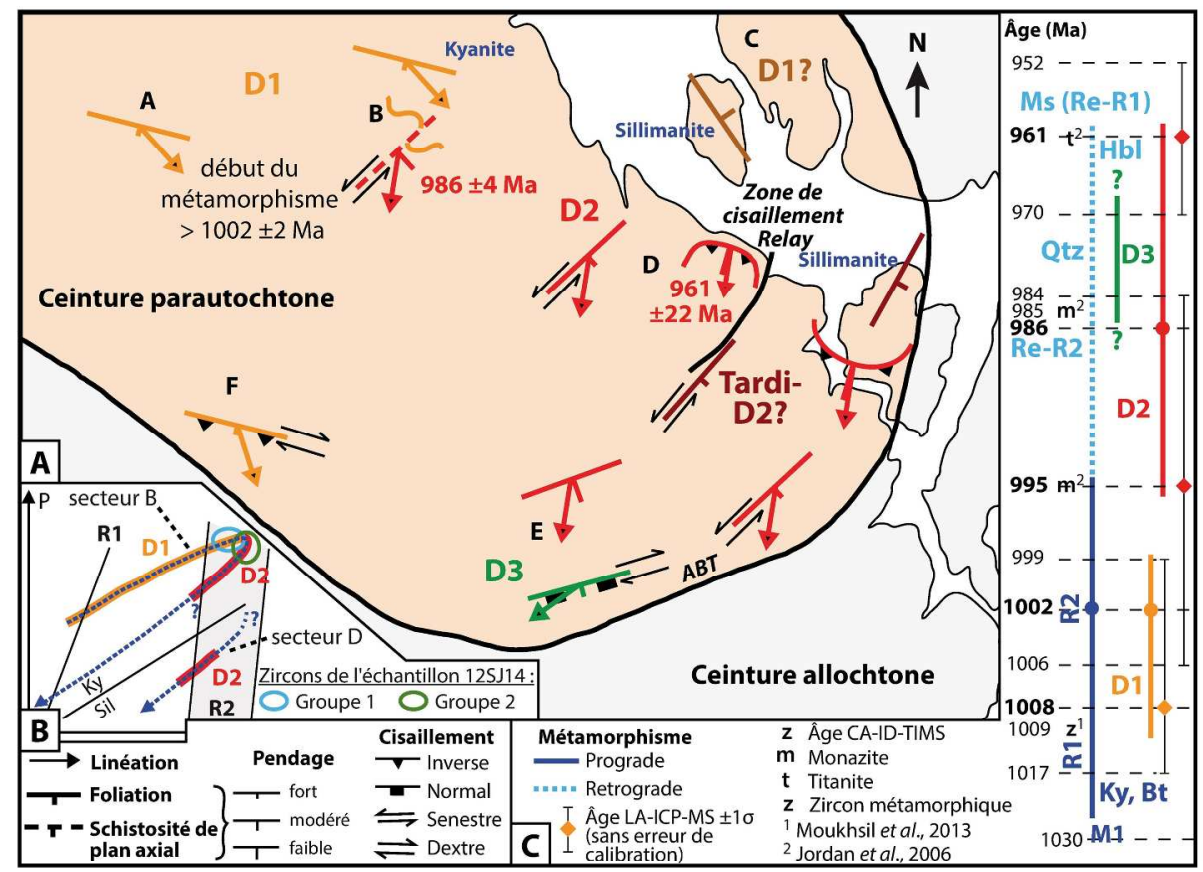

Figure 11 : A) Schéma illustrant les différentes phases de déformation et de métamorphisme avec leur âge dans la Ceinture parautochtone. B) Chemins P-T schématiques des secteurs B et $D$, inspirés de Jordan et al. (2006) illustrant la chronologie relative entre le métamorphisme, la déformation et la cristallisation des grains de zircon de l'échantillon 12SJ14. Puisque l'intervalle de température dans lequel la réaction R1 est continue est faible, cette réaction forme globalement une droite dans le champ P-T, alors que la réaction R2 est continue sur un intervalle de température plus grand et se déroule donc dans la zone illustrée en teinte de gris. C) Tableau illustrant l'évolution tectono-métamorphique de la Ceinture parautochtone (modifié de Jannin et al., accepté pour publication). 

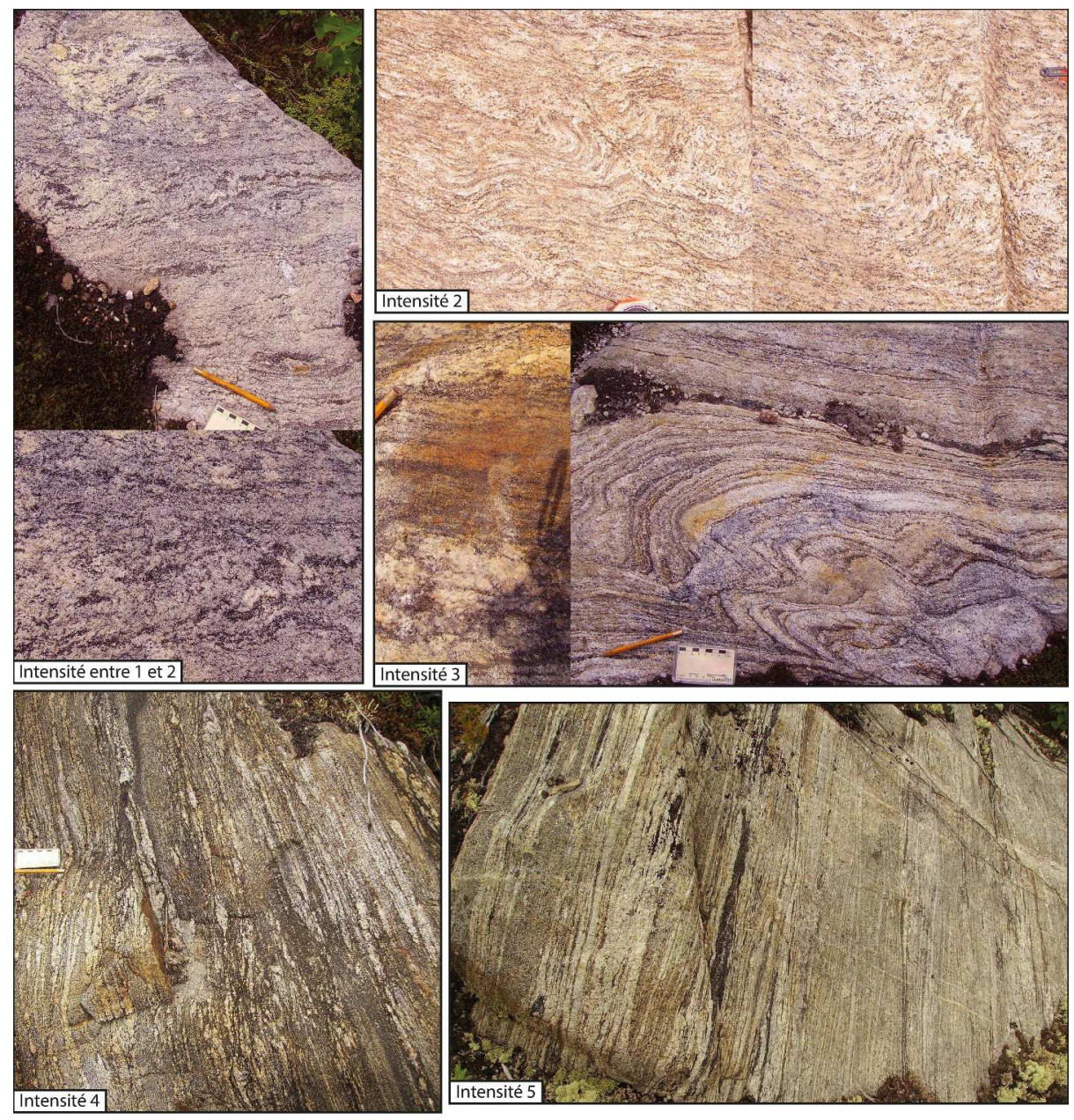

Figure A1: Illustration des valeurs de l'intensité de la déformation (voir le texte de l'appendice 1 pour les caractéristiques de chaque intensité; la valeur 5 correspond aux gneiss droits définis par Hanmer, 1988).

$236 \times 308 \mathrm{~mm}(300 \times 300 \mathrm{DPI})$ 